\title{
A Formal Approach to the Engineering of Domain-Specific Distributed Systems ${ }^{\text {th }}$
}

\author{
Rocco De Nicola ${ }^{\mathrm{a}, \mathrm{e}}$, Gianluigi Ferrari ${ }^{\mathrm{b}}$, Rosario Pugliese ${ }^{\mathrm{c}}$, Francesco Tiezzi ${ }^{\mathrm{d}}$ \\ ${ }^{a} I M T$ School for Advanced Studies Lucca, Italy \\ ${ }^{b}$ Università di Pisa, Italy \\ ${ }^{c}$ Università degli Studi di Firenze, Italy \\ ${ }^{d}$ Università di Camerino, Italy \\ ${ }^{e}$ National Cybersecurity Laboratory, CINI, Italy
}

\begin{abstract}
We review some results regarding specification, programming and verification of different classes of distributed systems which stemmed from the research of the Concurrency and Mobility Group at University of Firenze. More specifically, we examine the distinguishing features of network-aware programming, service-oriented computing, autonomic computing, and collective adaptive systems programming. We then present an overview of four different languages, namely Klaim, Cows, ScEL and AbC. For each language, we discuss design choices, present syntax and semantics, show how the different formalisms can be used to model and program a travel booking scenario, and describe programming environments and verification techniques.
\end{abstract}

Keywords: Coordination, Distributed Systems, Domain-Specific Languages

\section{Contents}

1 Introduction

2 KLAIM: Kernel language for Agents Interaction and Mobility 6

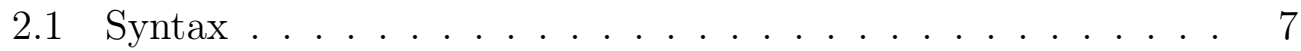

\footnotetext{
This work is a revised and extended version of [1]. It appeared in the Proceedings of the 20th International Conference on Coordination Models and Languages (COORDINATION2018).
} 
2.2 Informal semantics . . . . . . . . . . . . . . . 8

2.3 A taste of the operational semantics . . . . . . . . . . . 9

2.4 A travel booking scenario . . . . . . . . . . . . . . . . 12

2.5 Programming environment . . . . . . . . . . . . . . . 17

2.6 Verification techniques . . . . . . . . . . . . . . 17

2.7 Related work . . . . . . . . . . . . . . . . 21

3 Cows: Calculus for Orchestration of Web Services 22

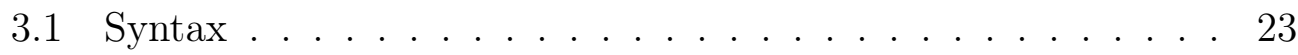

3.2 Informal semantics . . . . . . . . . . . . . . . . . 23

3.3 A taste of the operational semantics . . . . . . . . . . . . 25

3.4 A travel booking scenario . . . . . . . . . . . . . . . 27

3.5 Programming environment . . . . . . . . . . . . . 32

3.6 Verification techniques . . . . . . . . . . . . . . 33

3.7 Related work . . . . . . . . . . . . . . . . . . . . 34

4 SCEL: Software Component Ensemble Language 36

4.1 Syntax . . . . . . . . . . . . . . . . 37

4.2 Informal semantics . . . . . . . . . . . . . . 38

4.3 A taste of the operational semantics . . . . . . . . . . . . . . . 40

4.4 A travel booking scenario . . . . . . . . . . . . . . . 43

4.5 Programming environment . . . . . . . . . . . . . . . . . . 49

4.6 Verification techniques ................. . . . 49

4.7 Related work . . . . . . . . . . . . . . . . . 52

5 AbC: Attribute-based communication 52

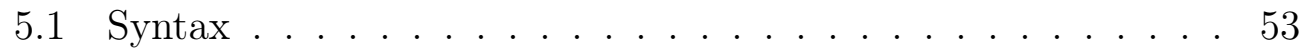

5.2 Informal semantics . . . . . . . . . . . . . . 54

5.3 A taste of the operational semantics . . . . . . . . . . . 55

5.4 A travel booking scenario . . . . . . . . . . . . . 58

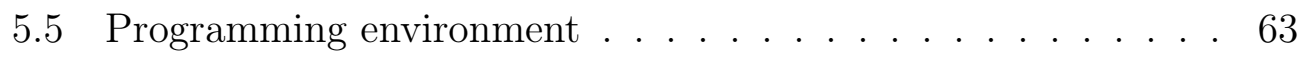

5.6 Verification techniques . . . . . . . . . . . . . 66

5.7 Related work . . . . . . . . . . . . . . . . . . 67

6 Concluding remarks $\quad 68$ 


\section{Introduction}

Since the mid-90s, we have witnessed an evolution of distributed computing towards increasingly complex systems formed by several software components featuring asynchronous interactions and operating in open-ended and non-deterministic environments. Such transformation, initially induced by the spreading of internetworking technologies, led to a paradigm shift making software components aware of the underlying network infrastructure. Network awareness, on the one hand, constrained the remote access to distributed resources and, on the other hand, enabled computation mobility, to support different kinds of optimisations.

On top of these networked systems, software components have been then deployed to provide services accessible by end-users and other system components through communication endpoints. This fostered the development of sophisticated applications built by reusing and composing simpler elements. Such service-based compositional approach abstracted from the actual distribution of the involved components over the underlying network, but required to deal with the interaction challenges posed by their heterogeneity. Interoperability was then achieved through the definition of standard protocols and suitable run-time support for programming languages that were taking into account also the failures that could occur in long-term interactions. Moreover, the absence of network awareness meant that there was no need for code mobility.

Later on, the need arose of reducing the maintenance cost of these webbased systems, whose size was becoming bigger and bigger, and of extending their applicability to interact with and control the physical world, possibly in scenarios where human intervention was difficult or even impossible. It was then advocated to rely on autonomic components, which are capable of continuously monitoring their internal status and the working environment, and to adapt their behaviour accordingly. In addition to point-to-point interactions, typical of client-server protocols, more sophisticated forms of interaction could occur that simultaneously involve an ensemble of components dynamically determined. Ensembles are to be intended as collections of taskoriented or dedicated components that pool their resources and capabilities together to create a more complex system, which offers more functionalities and higher performance than simply the sum of the constituent elements.

More recently, in some classes of autonomic computational systems we have witnessed the tremendous growth in the number of interacting compo- 
nents that are usually distributed, heterogeneous, decentralised and interdependent, and operate in dynamic and possibly unpredictable environments. The components form collectives by combining their behaviours to achieve specific goals or to contribute to an emerging behaviour of the global system. Collectives abstract from the identity of the single components to guarantee scalability.

The evolution of distributed computing described above corresponds to the emergence of classes of systems that characterise specific programming domains. Correspondingly, dedicated programming paradigms have been proposed, namely:

network-aware programming to exploit the knowledge of the underlying infrastructure for better using network facilities and moving programs closer to the resources they want to use [2];

service-oriented computing to allow the exploitation of loosely-coupled services as fundamental resources for developing applications and support the rapid and automatic development of open distributed systems [3];

autonomic computing to guarantee the self-managing characteristics of distributed computing resources, adapting to unpredictable changes while hiding intrinsic complexity to operators and users [4];

collective adaptive systems programming to model complex systems with large numbers of heterogeneous entities interacting without a specific central control, and adapting to environmental settings in pursuit of an individual or collective goal [5].

Besides dealing with the distinctive aspects of each of such domains, the main challenge in engineering these classes of distributed systems is to coordinate the overall behaviour resulting from the involved distributed components while ensuring trustworthiness of the whole system. To meet this goal, many researchers have adopted a language-based approach that combines the use of formal methods techniques with model-driven software engineering. The key ingredients of the resulting methodology, that can be applied to all classes of systems described above, may be summarised as relying on:

1. a specification language equipped with a formal semantics, which associates mathematical models to each term of the language to precisely establish the expected behaviour of systems; 
2. a set of techniques and tools, built on top of the models, to express and verify properties of interest;

3. a programming framework together with an associated runtime environment, to actually execute the specified systems.

When specialising this methodology, a major challenge for (specification or programming) language designers is to devise appropriate abstractions and linguistic primitives to deal with the specificities of the domain under investigation. Indeed, including the distinctive aspects of the domain as first-class elements of the language makes systems design more intuitive and concise, and their analysis more effective. In fact, when the outcome of a verification activity is expressed by considering the high level features of a system, and not its low-level representation, system designers can be provided with a more direct feedback.

This paper reviews some of the efforts, to which the authors have contributed, in applying the outlined methodology to the classes of distributed systems mentioned above by taking as starting point process algebras and some of the verification techniques and tools developed for them. The approach was initially applied to network-aware programming and the main result was the definition of the KLAIM language [6] that had explicit localities, processes mobility and network connections as primitive notions (Section 2). Afterwards, the approach was applied to service-oriented computing resulting in the design of Cows [7] whose basic constructs permitted to express correlations between clients and services and to deal with services failures. (Section 3). Instead, to deal with autonomic computing the SCEL language [8] was introduced that had explicit notions of agents knowledge and primitives and policies for its manipulations together with an original approach to ad hoc ensembles formation (Section 4). Finally, to model and prove emergent properties of collective adaptive systems a distilled version of SCEL named AbC [9] was introduced that had specific operators for selecting communication partners using predicates on the run time value of relevant attributes of the agents forming the system (Section 5).

In the following parts of this paper, for each of these domain-specific languages, we discuss the design choices behind it, present its syntax and informal semantics, and provide an excerpt of the rules defining its formal operational semantics in terms of labelled transition systems by relying on the Structural Operational Semantics style [10]. For each language, we also briefly describe the programming environments that have been developed 
to support program execution and outline some of the techniques that have been advocated for the verification of properties of the specified systems.

Moreover, to assess the expressive power of the different formalisms and to put them at work, we show how they can be used to model a simple scenario that is instrumental to highlight distinguishing features. For each formalism, we also provide some code snippets showing how close the specification of the model is to its underlying implementation. The scenario considers an online travel broker that, starting from specific requirements of customers, looks for hotel rooms and flights. Customers communicate their preferences to the broker and this, after some preliminary assessments, forwards the requirements to a number of hotels and air companies. Those, upon request, declare their availability and prices so that the customers can take the final choices and proceed with the booking.

The paper ends with a summary of distinguishing features of the presented languages and with a few considerations about the lessons learnt (Section 6).

\section{KLAIM: Kernel language for Agents Interaction and Mobility}

Network awareness indicates the ability of the software components of a distributed application to manage directly a sufficient amount of knowledge about the network environment where they are currently deployed. This capability allows components to have a highly dynamic behaviour and manage unpredictable changes of the network environment over time. This is of great importance when programming mobile components capable of disconnecting from one node of the underlying infrastructure and of reconnecting to a different node. Programmers are usually supported with primitive constructs that enable components to communicate, and to distribute and retrieve data to and from the nodes of the underlying infrastructure.

KLAim (Kernel Language for Agents Interaction and Mobility, [6]) has been specifically devised to design distributed applications consisting of several components, both stationary and mobile, deployed over the nodes of a distributed infrastructure. The KLAIM programming model relies on a unique interface (i.e. set of operations) supporting component communications and data management.

Localities are the basic building blocks of KLAIM for guaranteeing network awareness. They are symbolic addresses (i.e. network references) of nodes and are referred by means of identifiers. Localities can be exchanged among 
the computational components and are subjected to sophisticated scoping rules. They provide the naming mechanism to identify network resources and to represent the notion of administrative domain: computations at a given locality are under the control of a specific authority. This way, localities naturally support the programming of spatially distributed applications.

KLAIM builds on Linda's notion of generative communication through a single shared tuple space [11] and generalises it to multiple distributed tuple spaces. A tuple space is a multiset of tuples. Tuples are anonymous sequences of data items and are retrieved from tuple spaces by means of an associative selection. Interprocess communication occurs through asynchronous exchange of tuples via tuple spaces: there is no need for producers (i.e. senders) and consumers (i.e. receivers) of a tuple to synchronise.

The obtained communication model has a number of properties that make it appealing for distributed computing in general (see, e.g., [12, 13, 14, 15]). It supports time uncoupling (data life time is independent of the producer process life time), destination uncoupling (data producers do not need to know the future use or the final destination of the data) and space uncoupling (programmers need to know a single interface only to operate over the tuple spaces, regardless of the network node where the action will take place).

\subsection{Syntax}

The syntax of KLAIM is presented in Table 1. We assume existence of two disjoint sets: the set of localities, ranged over by $l$, and the set of locality variables, ranged over by $u$, with the distinguished variable self denoting the locality of the node using it. Their union gives the set of names, ranged over by $\ell$. We also assume three other disjoint sets: a set of value variables, ranged over by $x$, a set of process variables, ranged over by $X$, and a set of process identifiers, ranged over by $A$.

NETS are finite collections of nodes where processes and data can be placed. A computational node takes the form $l::_{\rho} P$, where $\rho$ is an allocation environment and $P$ is a process. Since processes may refer to locality variables, the allocation environment acts as a name solver binding locality variables to specific localities.

Processes are the active computational units of KLAIM. Each process is obtained by composing subprocesses or the inert process nil via action prefixing $($ a.P $)$, nondeterministic choice $\left(P_{1}+P_{2}\right)$, parallel composition $\left(P_{1} \mid P_{2}\right)$, process variable $(X)$, and parameterised process invocation $(A(\bar{p}))$. Recursive behaviours are modelled via process definitions; it is assumed that each 


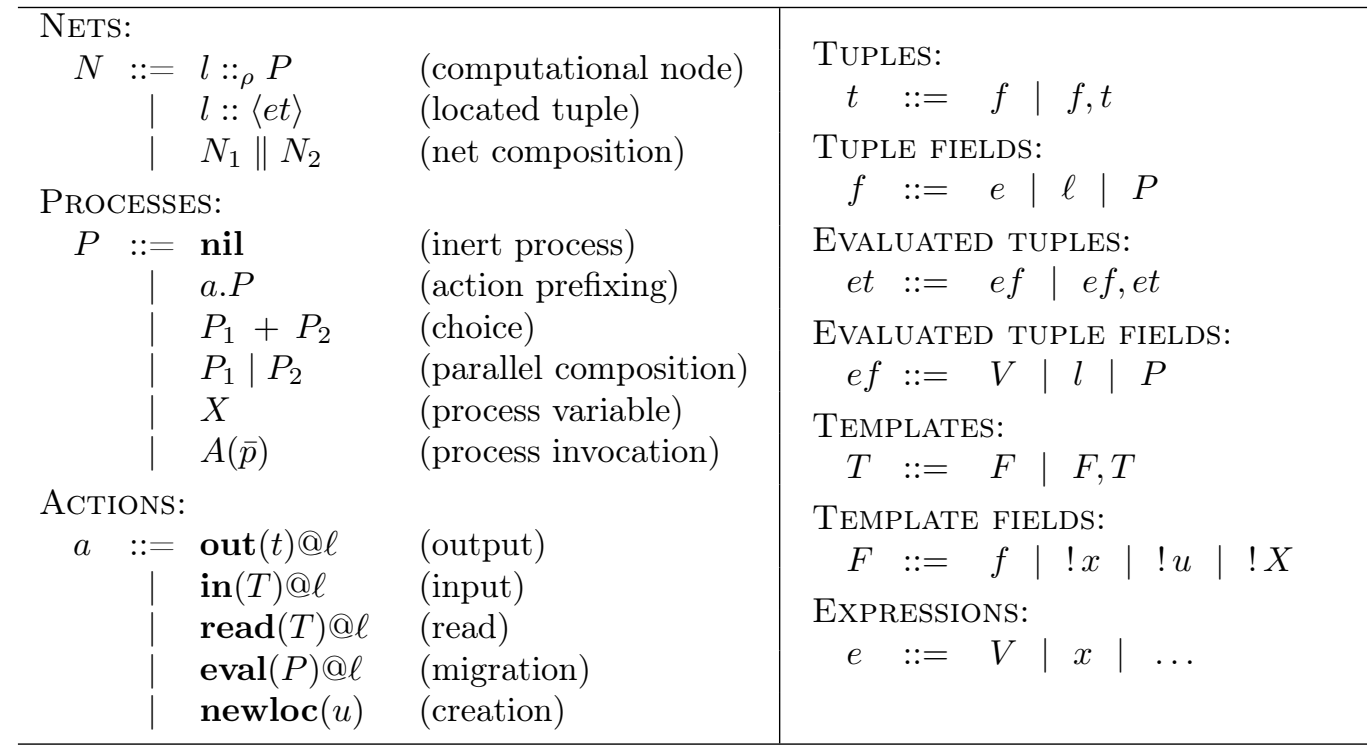

Table 1: Klaim syntax

identifier $A$ has a single defining equation $A(\bar{q}) \triangleq P$. Lists of actual and formal parameters are denoted by $\bar{p}$ and $\bar{q}$, respectively.

The tuple space of a node consists of all the EVALUATED TUPLES located there. TUPLES are sequences of actual fields, i.e. expressions, localities or locality variables, or processes. The precise syntax of EXPRESSIONS is deliberately not specified; it is just assumed that they contain, at least, basic values, ranged over by $V$, and value variables, ranged over by $x$. TEMPLATES are sequences of actual and formal fields, and are used as patterns to select tuples in a tuple space. Formal fields are identified by the !-tag (e.g. ! x) and are used to bind variables to values.

\subsection{Informal semantics}

NETS aggregate nodes through the composition operator $\|_{-}$, which is both commutative and associative. PROCESSES are concurrently executed in an interleaving fashion, either at the same computational node or at different nodes. They can perform operations borrowed from a unique interface which provides two categories of actions. The first one consists of the programming abstractions supporting data management. Three primitive behaviours are provided: adding (out), withdrawing (in) and reading (read) a tuple to/from a tuple space. Input and output actions are mutators: their exe- 
cution modifies the tuple space. The read action is an observer: it checks the availability and takes note of the content of a certain tuple without removing it from the tuple space. The second category of actions refers to network awareness: the migration action (eval) activates a new process over a network node, while the creation action (newloc) generates a new network node. The latter action is the only one not indexed by a locality because it acts locally; all the other actions are tagged with the (possibly remote) locality where they will take place. Note that, in principle, each network node can provide its own implementation of the action interface. This feature can be suitably exploited to sustain different policies for data handling as done, e.g., in MetaKlaim [16].

Only evaluated tuples can be added to a tuple space and templates must be evaluated before they can be used for retrieving tuples. Tuple and template evaluation amounts to computing the values of expressions and using the local allocation environment as a name solver for mapping locality variables to localities. As a consequence, the locality variables within processes in a tuple are mapped to localities by using the local allocation environment. Localities and formal fields are left unchanged by such evaluation. A pattern-matching mechanism is then used for associatively selecting (evaluated) tuples from tuple spaces according to (evaluated) templates.

Process variables support higher-order communication, namely the capability to exchange (the code of) a process and possibly execute it. This is realised by first adding a tuple containing the process to a tuple space and then retrieving/withdrawing this tuple while binding the process to a process variable.

Finally, KLAIM offers two forms of process mobility. One is based on static scoping: by exploiting higher-order communication, a process moves along the nodes of a net with a fixed binding of resources determined by the allocation environments of the nodes from where, from time to time, it is going to move. The other form of mobility relies on dynamic scoping: when migrating, a process breaks the local links to resources and inherits those of the destination node.

\subsection{A taste of the operational semantics}

The operational semantics is only defined for well-formed nets and it is given in terms of a structural congruence and a reduction relation over nets. A net is deemed well-formed if for each node $l::{ }_{\rho} P$ we have that $\rho$ (self) $=l$ and $f v(P) \subseteq \operatorname{dom}(\rho)$, and for any pair of nodes $l:: \rho P$ and $l^{\prime}:: \rho^{\prime} P^{\prime}$, we 
have that $l=l^{\prime}$ implies $\rho=\rho^{\prime}$. Notation $\operatorname{dom}(\rho)$ denotes the set of locality variables mapped by the allocation environment $\rho$, while $f v(P)$ denotes the set of free variables of process $P$. A variable is free in $P$ if it is not bound and it is bound in $P$ if it occurs within a formal field of $\operatorname{in}(T) @ \ell$ or $\operatorname{read}(T) @ \ell$, or is the argument $u$ of $\operatorname{newloc}(u)$; the scope of the binding is the process after the prefix. Actions out and eval are not binders, but their arguments may contain variables. For the sake of simplicity, we assume that, for the processes we consider, bound variables are all distinct and different from the free ones.

The structural congruence, $\equiv$, identifies syntactically different nets that intuitively represent the same net. It is defined as the smallest congruence relation over nets that satisfies a given set of laws. The most significant law is $l::_{\rho}\left(P_{1} \mid P_{2}\right) \equiv l:: P_{1} \| l \quad:{ }_{\rho} P_{2}$ meaning that it is always possible to transform a parallel of co-located processes into a parallel over nodes. The remaining laws express that $(i) \|$ is commutative and associative, $(i i)$ the inert process can always be safely removed/added, and (iii) a process identifier can be replaced with the body of its definition.

The reduction relations exploits two functions: one for evaluating tuples and templates, the other for selecting tuples in a tuple space. The evaluation function for tuples and templates takes as parameter the allocation environment of the node where the evaluation takes place. The main clauses of its definition are given below:

$$
\llbracket u \rrbracket_{\rho}=\left\{\begin{array}{ll}
\rho(u) & \text { if } u \in \operatorname{dom}(\rho) \\
\text { undef } & \text { otherwise }
\end{array} \llbracket P \rrbracket_{\rho}=P\{\rho\}\right.
$$

where $P\{\rho\}$ denotes the process term obtained from $P$ by replacing any free occurrence of a locality variable $u \in \operatorname{dom}(\rho)$ that is not within the argument of an eval with $\rho(u)$. Two examples of process evaluation are $\llbracket \operatorname{out}(P) @ \ell . Q \rrbracket_{\rho}=\operatorname{out}\left(\llbracket P \rrbracket_{\rho}\right) @ \rho(\ell) . Q\{\rho\}$ and $\llbracket \operatorname{eval}(P) @ \ell . Q \rrbracket_{\rho}=$ $\operatorname{eval}(P) @ \rho(\ell) . Q\{\rho\}$. We shall write $\llbracket t \rrbracket_{\rho}=$ et to denote that evaluation of tuple $t$ using $\rho$ succeeds and returns the evaluated tuple $e t$.

For selecting an evaluated tuple et from a tuple space according to an evaluated template ET, the pattern-matching function, match $(E T$, et $)=\sigma$, is used. This function is defined by means of a set of inference rules which intuitively state that: an evaluated template matches against an evaluated tuple if both have the same number of fields and corresponding fields do match; two values match only if they are identical, while formal fields match any value of the same type. A successful matching returns a substitution 


$$
\begin{aligned}
& \frac{\rho(\ell)=l^{\prime} \quad \llbracket t \rrbracket_{\rho}=e t}{l::_{\rho} \operatorname{out}(t) @ \ell . P\left\|l^{\prime}:: \rho_{\rho^{\prime}} P^{\prime} \succ l::_{\rho} P\right\| l^{\prime}:: \rho_{\rho^{\prime}} P^{\prime} \| l^{\prime}::\langle e t\rangle} \text { (OUT) }
\end{aligned}
$$

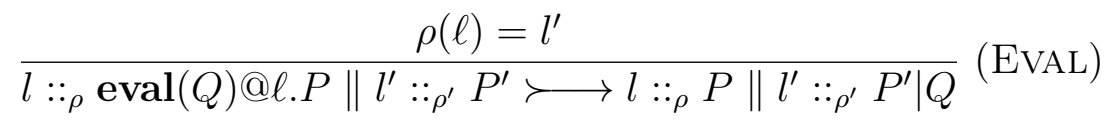

$$
\begin{aligned}
& \frac{\rho(\ell)=l^{\prime} \quad \operatorname{match}\left(\llbracket T \rrbracket_{\rho}, e t\right)=\sigma}{l::{ }_{\rho} \operatorname{in}(T) @ \ell . P\left\|l^{\prime}::\langle e t\rangle \succ:_{\rho} P \sigma\right\| l^{\prime}:: \text { nil }}(\mathrm{IN}) \\
& \frac{\rho(\ell)=l^{\prime} \quad \operatorname{match}\left(\llbracket T \rrbracket_{\rho}, e t\right)=\sigma}{l:: \rho \operatorname{read}(T) @ \ell . P\left\|l^{\prime}::\langle e t\rangle \succ:_{\rho} P \sigma\right\| l^{\prime}::\langle e t\rangle}(\mathrm{READ}) \\
& l^{\prime} \notin L \\
& L \vdash l::_{\rho} \operatorname{newloc}(u) . P \succ L \cup\left\{l^{\prime}\right\} \vdash l:_{\rho} P\left[l^{\prime} / u\right] \| l^{\prime}::_{\rho\left[l^{\prime} / \text { self }\right]} \text { nil }
\end{aligned}
$$

Table 2: KLAim operational semantics

function $\sigma$ associating the variables contained in the formal fields of the template with the values contained in the corresponding actual fields of the accessed tuple.

The reduction relation, $\succ \longrightarrow$, is defined as the least relation induced by a given set of inference rules. It is defined over configurations of the form $L \vdash N$, where $L$ is a finite set of localities keeping track of the localities occurring free in $N$ (that is, $f v(N) \subseteq L$ ). $L$ is needed to ensure global freshness of new (dynamically generated) network localities and is indeed omitted whenever a reduction does not generate any fresh locality.

The most significant rules are reported in Table 2 , where we write $\rho(\ell)=l$ to denote that either $\ell=l$ or $\ell$ is a locality variable that $\rho$ maps to $l$. In rule (OUT), the local allocation environment is used both to determine the name of the node where the tuple must be placed and to evaluate the argument tuple. This implies that if the argument tuple contains a field with a process $P$, the corresponding field of the evaluated tuple contains the process resulting from the evaluation of its locality variables, that is $P\{\rho\}$. Hence, processes in a tuple are transmitted after the interpretation of their free locality variables through the local allocation environment. This corresponds to having a static scoping discipline for the (possibly remote) generation of 
tuples. (OUT) requires existence of the target node at $l^{\prime}$, which is left unchanged by the reduction, and that the tuple $t$ argument of out is evaluable. As a result of the reduction, the tuple resulting from the evaluation of $t$ is added to the tuple space at $l^{\prime}$. A dynamic linking strategy is adopted for the eval operation, rule (EvAL). In this case the locality variables of the spawned process are not interpreted using the local allocation environment: the linking of locality variables is done at the remote node. The underlying assumption that all the equations for process definitions are available everywhere greatly simplifies rule (EVAL), because it permits avoiding mechanisms for code inspection to find the process definitions needed by $Q$. Rule (IN) requires that the template $T$ argument of in is evaluable and that a matching tuple at the target node exists. As a result of the reduction, the matched tuple is removed from the target tuple space and the substitution returned by the pattern-matching function is applied to the continuation of the process performing the action, in order to replace the free occurrences of the variables bound by $T$ with the corresponding values of et. Rule (READ) is similar, it only differs from (IN) just because the accessed tuple is still left in the tuple space. Finally, in rule (NEw), the premise exploits the set $L$ to choose a fresh locality $l^{\prime}$ for naming the new node. In the continuation of the process performing the action the locality variable $u$ argument of newloc is replaced by $l^{\prime}$, thus the new locality becomes usable for the process. Notably, $l^{\prime}$ is not yet known to any other node in the net. Hence, it can be used by the creating process as a private name. The allocation environment of the new node is derived from that of the creating one with the obvious update for the location variable self. Therefore, the new node inherits all the bindings of the creating node.

\subsection{A travel booking scenario}

We illustrate some of the distinguishing features of the KLAIM programming model by using the online travel booking scenario informally presented in the Introduction. The KLAIM specification consists of a collection of KLAIM nodes, each modelling a component of the software architecture of the scenario. For simplicity, we focus on three main components:

- the BROKER component where customers enter their requests, including the date and the origin-destination of the travel;

- the HOTEL and FLIGHT components that are in charge of selecting hotels and flights in compliance with customers' requests. 


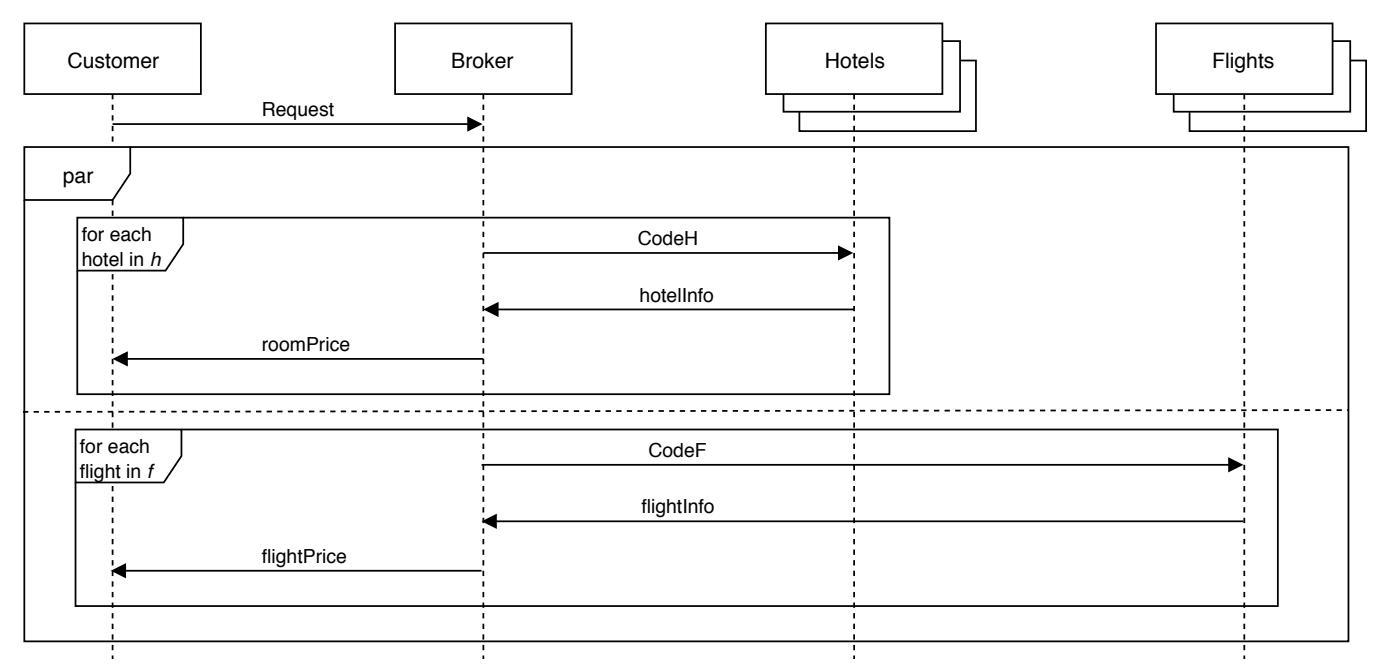

Figure 1: Travel Booking Scenario in KlaIm: Sequence Diagram.

The UML activity diagram displayed in Fig. 1 illustrates the flow of control inside the KLAIM net implementing the travel booking scenario. The BROKER, after collecting requests from customers, exploits code mobility to activate some spider processes in the HOTEL and FLIGHT nodes. These spider processes act on behalf of the BROKER to find hotels and flights matching customer's request. The exploitation of code mobility within the workflow of the application is expressed in the diagram by the CodeH (resp. CodeF) label. Once available, the result of the search carried out by the spider processes is communicated back to the BROKER.

When we presented the KLAIM programming model, we mainly focused on the linguistic primitives to structure distributed applications and to program behaviour. Indeed, we have deliberately not considered primitive data types. We now show how to equip KLAIM with simple data types. As an example, we introduce a data type for handling non-empty sequences of locations. We represent them through (the standard) square brackets commaseparated value notation: $\left[l_{1}, l_{2}, \ldots l_{k}\right]$. The unary function [] takes as input a location and yields as result the sequence consisting of the argument location only: []$(l)=[l]$. The binary function :: (read cons) takes as input a location and a sequence, and produces as result a new sequence whose first element is the argument location: $l::\left[l_{1}, l_{2}, \ldots l_{k}\right]=\left[l, l_{1}, l_{2}, \ldots l_{k}\right]$. Sequences now appear in tuples, hence pattern-matching has to be extended accordingly. For instance, the template $[! u]$ matches all sequences consisting of one location 
only. The template $! u:: ! s$ matches all sequences having at least two elements. Hereafter, we will apply pattern-matching to values in order to recognise the form of values and let the computation be guided accordingly. The same approach can be followed to include other data types, such as Strings, Dates, and so on.

The structure of the nodes where the hotel and flight facilities are deployed is intuitively clear. The node hosting the hotel booking facility is presented below:

$$
l_{h}: \rho_{\rho_{h}}\left(\text { HotelManager } \mid T_{h}\right)
$$

The node hosts the process HotelManager, which manages the hotel booking requests activated by the broker component. Moreover, it exposes room availability through suitable tuples stored in the local tuple space $T_{h}$.

The KLAIM node that specifies the behaviour of the BROKER component is as follows:

$$
l_{b r}: \rho_{b r}\left(\text { Handler } \mid \text { SessionManager } \mid T_{b r}\right)
$$

The node hosts the processes Handler and SessionManager presented below, together with the local tuple space, represented by $T_{b r}$. For the sake of readability, we exploit a sort of macro-like mechanism to associate a name to a piece of KLAIM specification, e.g. we write $A \equiv P$ to indicate that the code of the process $P$ will replace the identifier $A$ each time this is encountered in the KLAIM specification.

$$
\begin{aligned}
& \text { Handler } \triangleq \operatorname{in}(! \text { usr, !date, !origin, !dest, !res)@self. } \\
& \text { out("Manage", usr)@self.in(usr,!sid)@self. } \\
& \text { ((SpiderHotel( sid, date) | } \operatorname{read}(\text { usr, " } h ", \text { !hpref }) @ \text { self. } \\
& \text { ManageHotelPref (usr, sid, res, hpref)) } \\
& \text { | (SpiderFlight(sid, date, origin, dest) | } \operatorname{read(usr,~"f",~!fpref)@self.~} \\
& \text { ManageFlightPref (usr, sid, res, fpref)) } \\
& \text { | Handler) } \\
& \text { SessionManager } \triangleq \operatorname{in}(" M a n a g e ", \text { !usr }) @ \text { self.... } \\
& \text { out(usr, sessionId)@self. SessionManager }
\end{aligned}
$$

The handler process Handler receives the customer request, obtained by sensing in the tuple space the tuple containing the data about the customer code, the date-origin-destination of the travel, and the location of the node 
where the results of the request will be stored. The handler process then activates the manager process SessionManager, by emitting in the local tuple space a tuple tagged by "Manage", and gets the actual session identifier, by inspecting the tuple space. We abstract from the detailed description of process SessionManager, since it deals with some low-level computational aspects specific for the considered application, rather than taking care of coordinating activities. We just assume that the omitted code creates the unique session identifier for the customer's request, and associates to it the sequence of hotels and the sequence of airline companies that must be queried to satisfy the customer's request.

The handler process exploits two recursive processes to activate the spider (mobile) processes in charge of finding hotels and flights.

$$
\begin{aligned}
& \text { SpiderHotel }(i d, d) \triangleq \mathbf{i n}(i d, \text { " } h ",[! u]) @ \text { self. eval(CodeH)@u.nil } \\
& +\operatorname{in}(i d, \text { " } h ",[! u: ! ! s]) @ \text { self. eval }(\mathrm{CodeH}) @ u \text {. } \\
& \operatorname{out}(i d, \text { " } h ", s) @ \text { self. SpiderHotel }(i d, d) \\
& \text { CodeH } \equiv \operatorname{out}(" \text { check", } i d, d) @ \text { self. } \\
& \text { (in("avail", } i d, \text { !info)@self.out }(i d, \text { info }) @ l_{b r} \text {.nil } \\
& + \text { in("no-avail", } i d) @ \text { self. nil) } \\
& \text { SpiderFlight }(i d, d, \text { orn }, d s t) \triangleq \operatorname{in}(i d, \text { "f", [!u])@self. eval(CodeF }) @ u . n i l \\
& +\operatorname{in}(i d, \text { "f", [!u ::!s])@self. eval(CodeF)@u. } \\
& \operatorname{out}(i d, \text { "f", } s) @ \text { self. SpiderFlight }(i d, d, \text { orn, dst) } \\
& \text { CodeF } \equiv \text { out("check", id, d, orn, dst)@self. } \\
& \text { (in("avail", id, !info)@self. out }(i d, \text { info }) @ l_{b r} \text {.nil } \\
& + \text { in("no-avail", } i d) @ \text { self. nil) }
\end{aligned}
$$

The spider processes take fully advantage of KLAIM dynamic linking mobility through the eval primitive. This ensures that each spider will be spawned on the remote node without evaluating its locality variables according to the allocation environment of the broker component. This programming choice implies that when the mobile code will run in the remote node of the hotel (resp. of the flight), the location self will be bound to the actual address of the location where the hotel (resp. flight) component is deployed. The result of this search is then forwarded back to the tuple space of the broker. 
The last part of the behaviour of the broker consists in the management of the customer's preferences. This strongly depends on the data type used to store preferences. We outline the abstract specification of the facility matching customer's preferences with respect to the hotel information, as the treatment of flight information is similar. For simplicity, we assume that the hotel information is stored in tuples of the form (name, rate, hotelgroup). We also assume that the customer has a loyalty card for a specific group of hotels which is stored in the tuple space of the broker and that the customer will add a distinguished tuple to the tuple space of the broker to signal the termination of the hotel booking activity.

$$
\begin{aligned}
& \text { ManageHotelPref (usr, sid, res, hpref }) \triangleq \\
& \text { in(sid, (!name, !rate, !gruop))@self. } \\
& \text { out("hCheck",group=hpref)@self. } \\
& \text { ( in("hCheck", true)@self. } \\
& \text { out(sid,name,rate, "reduced-price")@res. } \\
& \text { ManageHotelPref (usr, sid, res, hpref) } \\
& \text { + in("hCheck", false)@self. } \\
& \text { out(sid,name, rate, "standard-price")@res. } \\
& \text { ManageHotelPref (usr, sid, res, hpref) ) } \\
& + \text { in(sid, "endHotelBooking")@self.nil }
\end{aligned}
$$

The process ManageHotelPref senses the local tuple space of the broker to identify the information about the hotels made available by the spider processes. This information is checked against the customer's preference (i.e., the hotel group) in order to report the presence of a reduced rate. Whenever information on the hotel meets the customer's preferences, the tuple containing the hotel data is stored in the remote tuple space of the customer with a flag indicating the availability of the reduced rate.

Discussion. The KLAIM primitive constructs for code mobility are instrumental to support the workflow of the travel booking scenario. We have seen that the spider processes exploit dynamic linking mobility. This has the additional benefit that the preferences associated with the specific customer are confined to the location of the broker. This is a simple way to obtain a suitable form of data privacy. More sophisticated forms of security could be obtained through the use of KLAIM types for access control or hierarchical KLAIM nets. We refer to [17] and to [18] for details. 


\subsection{Programming environment}

X-KLAim ${ }^{1}$ (eXtended KLAim, [19]) is an experimental programming language that extends KLAIM with a high level syntax for processes. It provides variable declarations, enriched operations, assignments, conditionals, sequential and iterative process composition. The implementation of X-KLAIM is based on KLAVA ${ }^{2}$ (KLAIM in Java, [20]), a Java package that provides the run-time system for X-KLAIM operations, and on a compiler, which translates X-KLAIM programs into Java programs that use KLAVA. A renewed and enhanced version of X-KLAIM is proposed in [21]. The new implementation comes together with an Eclipse-based IDE tooling, and relies on recent powerful frameworks for the development of programming languages, in particular the XTEXT framework [22].

X-KLAIM can be used to write the higher layer of distributed applications while KLAVA can be seen both as a middleware for X-KLAIM programs and as a Java framework for programming according to the KLAIM paradigm. By using KLAVA directly, the programmer is able to implement a finer grained type of mobility.

Fig. 2 lists a significant fragment of $\operatorname{code}^{3}$ of the X-KLAIM implementation of the KLAIM specification of the travel booking scenario, presented in Section 2.4. The X-KLAIM code permits appreciating how close a KLAIM specification is to its X-KLAIM implementation. Indeed, the syntax of the communication primitives is the same, except for the notation of formal fields that in X-KLAim are specified as (typed) variable declarations. Notably, concurrent subprocesses of the Handler process, composed by means of the | operator in KLAIM, are activated in X-KLAIM using the eval action with

target self. Finally, in the definition of the network, physical localities are expressed in terms of the standard TCP syntax host:port.

\subsection{Verification techniques}

Many verification techniques have been defined for KLAIM and variants thereof. Here we only mention a few of them. In [23] a temporal logic is proposed for specifying and verifying dynamic properties of mobile processes

\footnotetext{
${ }^{1}$ X-KLAIM is available online at https://github.com/LorenzoBettini/xklaim.

${ }^{2}$ KLAvA is available online at http://music.dsi.unifi.it.

${ }^{3}$ The X-Klaim source code for the complete scenario can be downloaded from https: //bitbucket.org/tiezzi/jlamp_survey_code/src/master/Klaim/.
} 


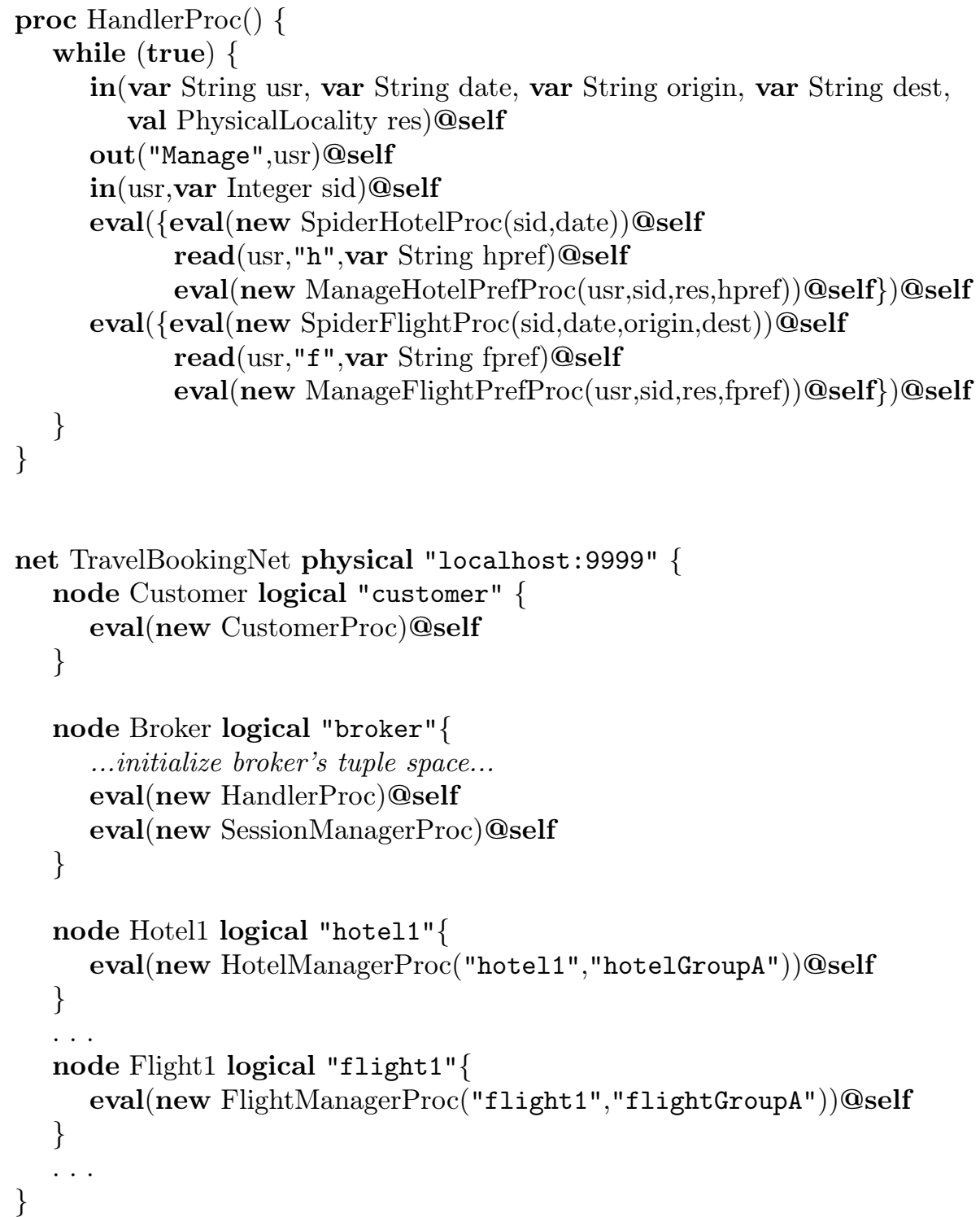

Figure 2: The process Handler and the network of the travel booking scenario implemented in X-KLAIM.

specified in KLAim. The inspiration for the proposal was the HennessyMilner Logic, but it needed significant adaptations due to the richer operating context of components. The resulting logic provides tools for establishing not only deadlock freedom, liveness and correctness with respect to given 
specifications (which are crucial properties for process calculi and similar formalisms), but also properties that relate to resource allocation, resource access and information disclosure (which are important issues for processes involving different actors and authorities).

An important topic deeply investigated for KLAIM is the use of type systems for security [17, 24, 25], devoted to control accesses to tuple spaces and mobility of processes. In these type systems, traditional types are generalised to behavioural types. These are abstractions of process behaviours that provide information about processes capabilities, namely the operations that processes can execute at a specific locality (downloading/consuming a tuple, producing a tuple, activating a process, and creating a new node). When using behavioural types, each KLAIM node is equipped with a security policy, determined by a net coordinator, that specifies the execution privileges; the policy of a node describes the actions processes there located can execute. By exploiting static and dynamic checks, type checking guarantees that only processes whose intentions match the rights granted to them by coordinators are allowed to proceed. An expressive language extension, called METAKLAIM, equipped with a powerful type system is described in [16]. MetaKLAim is a higher order distributed process calculus equipped with staging mechanisms. It integrates METAML (an extension of SML for multi-stage programming) and KLAIM, to permit interleaving of meta-programming activities (such as assembly and linking of code fragments), dynamic checking of security policies at administrative boundaries, and traditional computational activities on a wide area network (such as remote communication and code mobility). METAKLAIM exploits a powerful type system (including polymorphic types à la system F) to deal with highly parameterised mobile components and to dynamically enforce security policies: types are metadata that are extracted from code at run-time and are used to express trustiness guarantees. The dynamic type checking ensures that the trustiness guarantees of wide area network applications are maintained also when computations interoperate with potentially untrusted components.

An alternative approach to control accesses to tuple spaces and mobility of processes is introduced in [26]. It is based on Flow Logic and permits statically checking absence of violations. Starting from an existing type system for KLAIM with some dynamic checks, the insights from the Flow Logic approach are exploited to construct a type system for statically guaranteeing secure access to tuple spaces and safe process migration for a smooth extension of KLAIM. This is the first completely static type system for con- 
trolling accesses devised for a tuple space-based coordination language. A static control flow analysis that extends the one proposed in [27, 28], to manage network awareness and coordination via multiple tuple spaces has been introduced in [29]. The static analysis can be used to detect where and how tuples are manipulated and how messages flow among the nodes of a KLAIM network. This permits to identify possible security breaches in the data workflow of a distributed application. For instance, it may keep the safe paths that data inside a tuple can traverse apart from those that pass through a possible untrusted node.

We now outline how the static methodology presented in [29] can be applied to investigate the security of KLAIM code. We illustrate this by resorting to the KLAIM specification of the travel booking scenario. The static methodology enables us to construct an abstract graph-based model of the behaviour of the KLAIM specification of the scenario. This abstract model supports a reasoning technique which permits to detect $(i)$ the path in the network through which (a value in) a tuple of a specific node reaches another one, and $(i i)$ the transformations which are applied to a selected value along those paths.

In the travel booking scenario, the abstract model approximates the trajectories of each piece of data. For instance, the abstract trajectory below

where

$$
\operatorname{Trajectory}\left(d_{0}, d_{f}\right)=l_{u}, d_{0}: l_{b r}, d_{1}: l_{h}, d_{2}: l_{b r}, d_{3}: l_{u}, d_{f}
$$

$$
\begin{aligned}
& d_{0}=\langle\text { customer, date, origin, dest }\rangle \quad \text { Customer Request } \\
& d_{f}=\text { bookingData Result of the search }
\end{aligned}
$$

expresses the path of the value bookingData associated to the customer's request. This trajectory, made of pairs of the form location, datum separated by the symbol ':', encodes the data transformations generated by each of the involved components in processing the customer's request together with the sequence of locations traversed due to the computation steps.

The abstract path above describes the capacity of the KLAIM code to correctly manage customer's request. Instead, the following abstract path $l_{u}, d_{0}: l_{b r}, d_{1}: l_{h}, d_{2}: l_{u}, d_{f}$ detects a suspicious trajectory, namely a trajectory that by-passes the phase where the results of the spider processes are collected together. More generally, by analysing the abstract paths derived from the model it is possible to identify crucial code structures. We refer to $[29,30]$ for more details. 


\subsection{Related work}

Especially at the beginning of this century, with the manifest pervasivity of the Internet, many researchers have considered both models and implementations of network-aware formalisms that have or have been influenced by the work on KLAIM and other Linda-based models and primitives. In [31], many implementations of Linda-based models, including KLAIM-based ones, to coordinate the interactions among system components are described and their efficiency is assessed. Instead, [32] is a recent survey of coordination techniques for distributed and mobile systems, including those based on Linda and those relying on different coordination models. For references on network-aware programming and relation with KLAIM we refer the interested reader to $[6,33]$.

Among the foundational calculi aiming at capturing the key notions of network-awareness and identifying the programming abstractions most suitable for network-aware programming, we would like to mention three different ones, namely the Distributed $\pi$-calculus (D $\pi$ ) [34], the Distributed Join Calculus (DJoin) [35, 36], the Ambient Calculus (Amb) [37], that were essentially proposed at the same time as KLAIM.

$D \pi$ is a variant of the $\pi$-calculus enriched with explicit locations that are used to distribute processes. Interprocess communication is binary, channelbased, synchronous and local, in the sense that only processes at the same location can exchange messages. A process willing to communicate with a remote one has first to migrate to its location.

In DJoin, a location is structured as a tree composed by the root location and its sub-locations. When a process defined at a specific location moves to a different location, the whole tree moves along with the process. Again, process communication is channel-based and there is a unique process that can receive on each channel. To synchronise, processes rely on so-called join patterns that may require pattern matching on data and simultaneous reception of messages on different channels.

Finally, in the Ambient calculus, the key notion is that of ambient that can be thought of as a bounded environment where processes cooperate. An ambient is characterised by a name, a collection of local agents and a collection of sub-ambients, and can be referred only through explicit naming. An agent moves together with the ambient containing it. Communication is local to ambients and takes place through anonymous message exchange, without resorting to channels or pattern matching. 


\section{Cows: Calculus for Orchestration of Web Services}

Since the early 2000s, the increasing success of e-business, e-learning, e-government, and other similar systems, has led the World Wide Web, initially thought of as a system for human use, to evolve towards an architecture for Service-Oriented Computing (SOC) supporting automated use. The SOC paradigm, that finds its origin in object-oriented and componentbased software development, aims at enabling developers to build networks of distributed, interoperable and collaborative applications, regardless of the platform where the applications run and of the programming language used to develop them. The paradigm is based on the use of independent computational units, called services. They are loosely coupled reusable components, that are built with little or no knowledge about clients and about other services involved in their operating environment.

One successful instantiation of the general SOC paradigm is given by the Web Service technology [38], which exploits the pervasiveness of the Internet and related standards. Traditional software engineering technologies, however, do not neatly fit with SOC, thus hindering its full realisation in practice. The challenges come from the necessity of dealing at once with such issues as asynchronous interactions, concurrent activities, workflow coordination, business transactions, resource usage, and security, in a setting where demands and guarantees can be very different for the many involved components.

Cows (Calculus for Orchestration of Web Services, [39, 7]) is a formalism whose design has been influenced by the OASIS standard WS-BPEL [40] for orchestration of web services. In Cows, services are computational entities capable of generating multiple instances to concurrently handle different client requests. Inter-service communication occurs through communication endpoints and relies on pattern-matching for logically correlating messages to form an interaction session by means of their identical contents. Differently from most process calculi, and from KLAIM, receive activities in Cows bind neither names nor variables, and this is crucial for allowing concurrent service instances to share (part of) the state. The calculus also supports service fault and termination handling by providing activities to force termination

of labelled service instances and to protect service activities from a forced termination. 


\begin{tabular}{|c|c|c|c|}
\hline \multirow{2}{*}{\multicolumn{3}{|c|}{$\begin{array}{l}\text { SERVICES: } \\
s \quad::=u \cdot u^{\prime} ! \bar{\epsilon}\end{array}$}} & \multirow{7}{*}{ 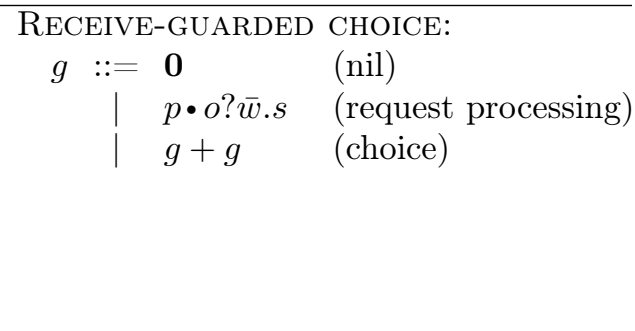 } \\
\hline & $u \bullet u^{\prime} ! \bar{\epsilon}$ & (invoke) & \\
\hline & $\operatorname{kill}(k)$ & (kill) & \\
\hline & & (receive-guarded choice) & \\
\hline & $s \mid s$ & (parallel composition) & \\
\hline & & $\begin{array}{l}\text { (protection) } \\
\text { (delimitation) }\end{array}$ & \\
\hline & $* s$ & (replication) & \\
\hline
\end{tabular}

Table 3: Cows syntax

\subsection{Syntax}

The syntax of Cows is presented in Table 3. We use three countable disjoint sets: the set of values (ranged over by $v$ ), the set of 'write once' variables (ranged over by $x$ ), and set of killer labels (ranged over by $k$ ). The set of values is left unspecified; however, we assume that it includes the set of partner and operation names (ranged over by $n, p, o$ ) mainly used to represent communication endpoints. We also use a set of expressions (ranged over by $\epsilon$ ), whose exact syntax is deliberately omitted; we just assume that expressions contain values and variables, and do not contain killer labels. As a matter of notation, $w$ ranges over values and variables, $u$ ranges over names and variables, and $e$ ranges over elements, i.e. killer labels, names and variables. Notation ${ }^{-}$stands for tuples, e.g. $\bar{x}$ means $\left\langle x_{1}, \ldots, x_{n}\right\rangle$ (with $n \geq 0$ ), where variables in the same tuple are all distinct.

Services are structured activities built from basic activities, i.e. the empty activity $\mathbf{0}$, the invoke activity ${ }_{-}{ }_{-} !_{-}$, the receive activity ${ }_{-}{ }_{-}{ }_{-}$, and the kill activity kill(_), by means of prefixing _.$_{-}$, choice ${ }_{-}+_{-}$, parallel composition _ $\left.\right|_{-}$, protection $\left\{\left.\right|_{-}\right\}$, delimitation [_] $]_{-}$and replication $*_{-}$. We write $I \triangleq s$ to assign a name $I$ to the term $s$.

\subsection{Informal semantics}

Invoke and receive are the communication activities. The former permits invoking an operation (i.e., a functionality like a method in object-oriented programming) offered by a service, while the latter permits waiting for an invocation to arrive. Besides output and input parameters, both activities indicate an endpoint through which communication should occur.

An endpoint $p \bullet o$ can be interpreted as a specific implementation of operation $o$ provided by the service identified by the logic name $p$. The names composing an endpoint can be dealt with separately, as in an asynchronous 
request-response interaction, where usually the service provider statically knows the name of the operation for sending the response, but not the partner name of the requesting service it has to reply to. Partner and operation names can be exchanged in communication, thus enabling many different interaction patterns among service instances. However, dynamically received names cannot form the endpoints used to receive further invocations (as in localised $\pi$-calculus [41]). In other words, endpoints of receive activities are identified statically because the syntax only allows using names and not variables for them. This design choice reflects the current (web) service technologies that require endpoints of receive activities to be statically determined.

An invoke $p \bullet o !\left\langle\epsilon_{1}, \ldots, \epsilon_{n}\right\rangle$ can proceed as soon as all expression arguments are successfully evaluated. A receive $p \bullet o ?\left\langle w_{1}, \ldots, w_{n}\right\rangle . s$ offers an invocable operation $o$ along with a given partner name $p$, thereafter the service continues as $s$. An inter-service communication between these two activities takes place when the tuple of values $\left\langle v_{1}, \ldots, v_{n}\right\rangle$, resulting from the evaluation of the invoke argument, matches the template $\left\langle w_{1}, \ldots, w_{n}\right\rangle$ argument of the receive. This causes a substitution of the variables in the receive template (within the scope of variables declarations) with the corresponding values produced by the invoke.

Communication is asynchronous, as in KLAIM. This results from the syntactic constraints that invoke activities cannot be used as prefixes and choice can only be guarded by receive activities (as in asynchronous $\pi$-calculus [42]). Indeed, in service-oriented systems, communication is usually asynchronous, in the sense that (i) there may be an arbitrary delay between the sending and the receiving of a message, (ii) the order in which messages are received may differ from that in which they were sent, and (iii) a sender cannot determine if and when a sent message will be received.

The empty activity does nothing, while choice permits selecting for execution one between two alternative receives.

Execution of parallel services is interleaved. However, if more matching receives are ready to process a given invoke, only one of the receives that generate a substitution with smallest size (in terms of number of variablevalue replacements) is allowed to progress (namely, execution of this receive takes precedence over that of the others). This mechanism permits to model the precedence of a service instance over the corresponding service specification when both of them can process the same request, and enables a sort of blind-date conversation joining strategy [43].

Delimitation is the only binding construct: $[e] s$ binds the element $e$ in 
the scope $s$. According to its first argument, delimitation is used for three different purposes: (i) to regulate the range of application of substitutions produced by communication, when the delimited element is a variable; (ii) to generate fresh names, when the delimited element is a name; (iii) to confine the effect of a kill activity, when the delimited element is a killer label. The scope of names can be dynamically extended, in order to model the communication of private names, as done with the restriction operator in $\pi$ calculus [44]. Instead, killer labels cannot be dynamically extended, because the activities whose termination would be forced by the execution of a kill need to be statically determined.

The kill activity forces immediate termination of all the concurrent activities not enclosed within the protection operator. To faithfully model fault and termination handling of SOC applications, kill activities are executed eagerly with respect to the communication activities enclosed within the delimitation of the corresponding killer label.

Finally, the replication construct $* s$ permits to spawn in parallel as many copies of $s$ as necessary. This, for example, is exploited to implement recursive behaviours and to model business process definitions, which can create multiple instances to serve several requests simultaneously.

\subsection{A taste of the operational semantics}

The operational semantics of Cows is defined only for closed services, i.e. services without free variables and killer labels. As usual, the semantics is formally given in terms of a structural congruence and of a labelled transition relation. The former identifies syntactically different services that intuitively represent the same service. Its definition is standard, except for the scope extension laws that permit to extend the scope of names (as in the $\pi$-calculus) and variables, thus enabling possible communication, but prevent extending the scope of killer labels.

We report in Table 4 an excerpt of the operational rules defining the labelled transition relation. We comment on the rules below.

A service invocation can proceed only if the expressions in the argument can be evaluated (rule (inv)). To this aim, we use the evaluation function

【_』that takes a closed expression and returns the corresponding value. This function is not explicitly defined, since the exact syntax of expressions is deliberately not specified. A receive activity offers an invocable operation along a given partner name (rule (rec)). Communication can take place when two parallel services perform matching receive and invoke activities 


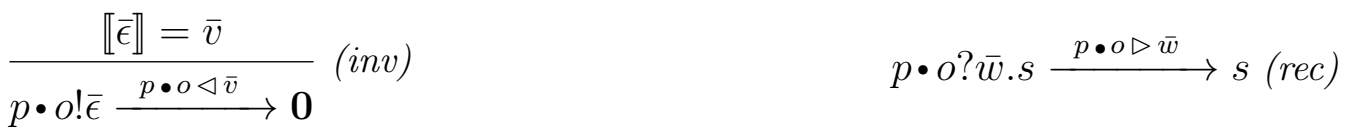

$$
\begin{aligned}
& \frac{s_{1} \stackrel{p \bullet o \triangleright \bar{w}}{\longrightarrow} s_{1}^{\prime} \quad s_{2} \stackrel{p \bullet o \triangleleft \bar{v}}{\longrightarrow} s_{2}^{\prime} \quad \mathcal{M}(\bar{w}, \bar{v})=\sigma}{s_{1}\left|s_{2} \stackrel{\sigma}{\longrightarrow} s_{1}^{\prime}\right| s_{2}^{\prime}} \text { (com) } \\
& \frac{s \stackrel{\sigma \uplus\{x \mapsto v\}}{\longrightarrow} s^{\prime}}{[x] s \stackrel{\sigma}{\longrightarrow} s^{\prime} \cdot\{x \mapsto v\}}\left(\text { del }_{\text {com }}\right) \quad \frac{s \stackrel{\alpha}{\longrightarrow} s^{\prime}}{\{|s|\} \stackrel{\alpha}{\longrightarrow}\left\{\left|s^{\prime}\right|\right\}} \text { (prot) } \\
& \operatorname{kill}(k) \stackrel{k}{\longrightarrow} \mathbf{0} \text { (kill) } \\
& \frac{s_{1} \stackrel{k}{\longrightarrow} s_{1}^{\prime}}{s_{1}\left|s_{2} \stackrel{k}{\longrightarrow} s_{1}^{\prime}\right| \operatorname{halt}\left(s_{2}\right)}\left(\operatorname{par}_{k i l l}\right)
\end{aligned}
$$

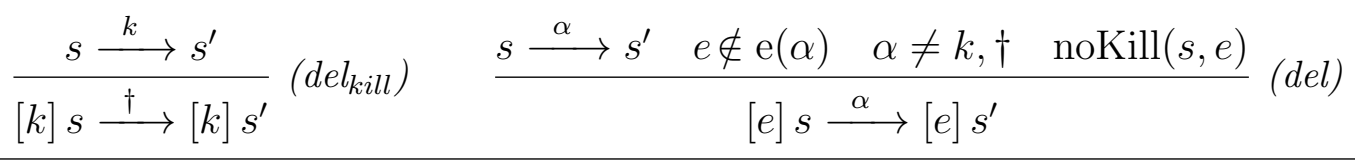

Table 4: Cows operational semantics (selected rules)

(rule $($ com $))$. We use here the partial function $\mathcal{M}\left({ }_{-},{ }_{-}\right)$for performing pattern-matching on semi-structured data (à la KLAIM). Pattern-matching permits to determine if a receive and an invoke over the same endpoint can synchronise. When tuples $\bar{w}$ and $\bar{v}$ do match, $\mathcal{M}(\bar{w}, \bar{v})$ returns a substitution $\sigma$ for the variables in $\bar{w}$; otherwise, it is undefined. Substitutions are functions mapping variables to values and are written as collections of pairs of the form $x \mapsto v$. Application of substitution $\sigma$ to $s$, written $s \cdot \sigma$, has the effect of replacing every free occurrence of $x$ in $s$ with $v$, for each $x \mapsto v \in \sigma$. The label of a communication transition indicates the generated substitution (for subsequent application), rather than a silent action as in most process calculi. When the delimitation of a variable $x$ argument of a receive involved in a communication is encountered, i.e. the whole scope of the variable is determined, the delimitation is removed and the substitution for $x$ is applied to the term (rule $\left(d_{e l} l_{c o m}\right)$ ). Variable $x$ disappears from the term and cannot be reassigned a value (for this reason Cows's variables are deemed 'write once'). We use $\sigma_{1} \uplus \sigma_{2}$ to denote the union of substitutions $\sigma_{1}$ and $\sigma_{2}$ when they have disjoint domains. 
Execution of parallel services is interleaved but, if more matching receives are ready to process a given invoke, only one of the receives that generate a substitution with smallest size (in terms of number of variable-value replacements) is allowed to progress (namely, execution of this receive takes precedence over that of the others). This mechanism permits to model the precedence of a service instance over the corresponding service specification when both of them can process the same request (we refer to [7, Sec. 3.2] for a complete account on this feature), and enables a sort of blind-date conversation joining strategy [43]. For the sake of presentation, we have omitted here this precedence mechanism, thus presenting a simplified version of the operational rules concerning the parallel composition operator.

Activity $\mathbf{k i l l}(k)$ forces termination of all unprotected parallel activities (rules (kill) and $\left(\right.$ par $\left._{\text {kill }}\right)$ ) inside the innermost enclosing $[k]$. Termination of a service $s$ is achieved by means of function halt $(s)$, which returns the service obtained by only retaining the protected activities inside $s$. The delimitation $[k]$ stops the killing effect by turning the transition label $k$ into $\dagger\left(\operatorname{rule}\left(\right.\right.$ del $\left.\left._{k i l l}\right)\right)$. Such delimitation, whose existence is ensured by the assumption that the semantics is only defined for closed services, prevents a single service to be capable to stop all the other parallel services, which would be unreasonable in a service-oriented setting (as services are loosely coupled and organized in different administrative domains). Critical activities can be protected from killing by putting them into a protection $\left\{\left.\right|_{-} \mid\right\}$; this way, $\{|s|\}$ behaves like $s$ (rule (prot)). Similarly, $[e] s$ behaves like $s$ (rule (del)), except when the transition label $\alpha$ contains $e$, in which case $\alpha$ must correspond either to a communication assigning a value to $e\left(\right.$ rule $\left(\right.$ del $\left.\left._{\text {com }}\right)\right)$ or to a kill activity for $e\left(\right.$ rule $\left(\right.$ del $\left.\left._{k i l l}\right)\right)$, or when a free kill activity for $e$ is active in $s$, in which case only actions corresponding to kill activities can be executed. Predicate $\operatorname{noKill}(s, e)$ is used to check the absence of a free kill activity: it holds true if either $e$ is not a killer label, or $e=k$ and $s$ cannot immediately perform a free kill activity $\operatorname{kill}(k)$. In this way, kill activities are executed eagerly with respect to the activities enclosed within the delimitation of the corresponding killer label.

\subsection{A travel booking scenario}

We provide here, in an incremental way, the Cows specification of our travel brokering scenario.

At a high level of abstraction, the travel broker service is rendered in 
Cows as:

$$
\begin{array}{r}
\text { Broker } \triangleq *\left[x_{\text {cust }}, x_{\text {dates }}, x_{\text {dest }}\right] p_{\text {br }} \bullet o_{\text {req }} ?\left\langle x_{\text {cust }}, x_{\text {dates }}, x_{\text {dest }}\right\rangle . \\
x_{\text {cust }} \bullet o_{\text {resp }} !\left\langle\operatorname{book}\left(x_{\text {dates }}, x_{\text {dest }}\right)\right\rangle
\end{array}
$$

The replication operator $*$ is used here to specify that the service is persistent, i.e. capable of creating multiple instances to serve several requests simultaneously. The delimitation operator specifies the scope of the variables arguments of the subsequent receive activity on operation $o_{r e q}$, used to receive a request message from a customer. Besides dates and destination of the travel, this message contains the partner name that the customer will use to receive the response, which will be sent by the service by means of the invoke activity on operation $o_{\text {resp }}$. Booking of hotel and flight is here abstracted by the (unspecified) expression book $\left(x_{\text {dates }}, x_{\text {dest }}\right)$.

A customer of the broker service is specified as follows:

$$
\text { Customer } \triangleq p_{\text {br }} \bullet o_{\text {req }} !\left\langle p_{c}, v_{\text {dates }}, v_{\text {dest }}\right\rangle \mid\left[x_{\text {travel }}\right] p_{c} \bullet o_{\text {resp }} ?\left\langle x_{\text {travel }}\right\rangle . s
$$

The customer behaviour is specular to that of the broker: it starts with an invoke and then waits for a response message containing the travel data.

The overall specification of the scenario is simply the parallel composition of the two components: (Customer | Broker). Whenever prompted by a client request, the broker service creates an instance to serve that specific request, and is immediately ready to concurrently serve other possible requests. Therefore, the resulting Cows term after such a computational step is the following:

$$
\left[x_{\text {travel }}\right] p_{c} \bullet o_{\text {resp }} ?\left\langle x_{\text {travel }}\right\rangle . s \mid \text { Broker } \mid p_{c} \bullet o_{\text {resp }} !\left\langle\operatorname{book}\left(v_{\text {dates }}, v_{\text {dest }}\right)\right\rangle
$$

The created service instance (highlighted by a grey background) is represented as a service running in parallel with the other terms. Notably, the variables of the invoke activity are instantiated (i.e., replaced) by the corresponding values exchanged in the communication. This invoke activity can now synchronise with the receive activity of the customer, whose execution will then continue as $s$ with $x_{\text {travel }}$ replaced by the value resulting from the evaluation of the book expression.

Let us now consider a more refined specification, where the role of the book expression is played by the interactions with services for flights and hotels searching. The interactions between a customer, the (refined) broker 


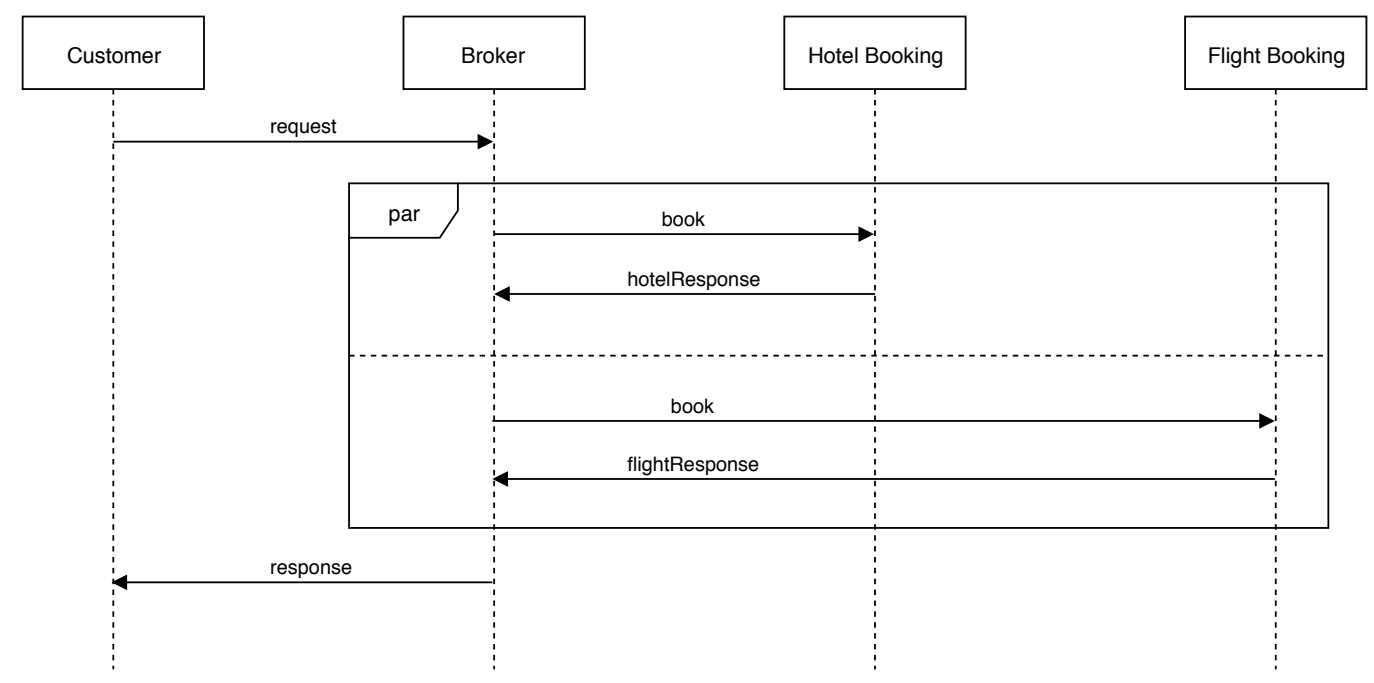

Figure 3: Travel Booking Scenario in Cows: Sequence Diagram.

and the searching services are described by the UML activity diagram in Fig. 3. The figure highlights that the broker service interacts in parallel with the flights and hotels searching services, and that it replies to the customer after both parallel interactions complete. The refined specification of the broker is the following:

$$
\begin{aligned}
\text { Broker }^{\prime} \triangleq * & {\left[x_{\text {cust }}, x_{\text {dates }}, x_{\text {dest }}\right] p_{\text {br }} \bullet o_{\text {req }} ?\left\langle x_{\text {cust }}, x_{\text {dates }}, x_{\text {dest }}\right\rangle . } \\
& {\left[p, o, x_{\text {flight }}, x_{\text {hotel }}\right] } \\
& \left(\mid\left(p_{\text {flight }} \bullet o_{\text {book }} !\left\langle p_{\text {br }}, x_{\text {cust }}, x_{\text {dates }}, x_{\text {dest }}\right\rangle\right.\right. \\
& \left.\mid p_{\text {br }} \bullet o_{\text {fRes }} ?\left\langle x_{\text {cust }}, x_{\text {dates }}, x_{\text {dest }}, x_{\text {fight }}\right\rangle .\left(p \bullet o !\langle\text { end }\rangle \mid s_{f}\right)\right) \\
& \mid\left(p_{\text {hotel }} \bullet o_{\text {book }} !\left\langle p_{\text {br }}, x_{\text {cust }}, x_{\text {dates }}, x_{\text {dest }}\right\rangle\right. \\
& \left.\mid p_{\text {br }} \bullet o_{\text {hRes }} ?\left\langle x_{\text {cust }}, x_{\text {dates }}, x_{\text {dest }}, x_{\text {hotel }}\right\rangle .\left(p \bullet o !\langle\text { end }\rangle \mid s_{h}\right)\right) \\
& \left.\mid p \bullet o ?\langle\text { ond }\rangle \cdot p \bullet o ?\langle\text { end }\rangle . x_{\text {cust }} \bullet o_{\text {resp }} !\left\langle x_{\text {fight }}, x_{\text {hotel }}\right\rangle\right)
\end{aligned}
$$

After the reception of a customer request, the service contacts in parallel the two searching services (by invoking the operation $o_{b o o k}$ ). When the responses from both services are available, the broker service combines them and replies to the customer. To this aim, a private endpoint $p \bullet o$ is exploited: the reception of a message from a searching service triggers an end signal (i.e., an internal message) along the private endpoint, and two of such signals are necessary to trigger the invoke activity for replying to the customer. Suitable expression functions could be used in this last invoke activity for filtering the 
results produced by the searching services. Notice that the scope of variable $x_{\text {flight }}\left(\right.$ resp. $x_{\text {hotel }}$ ) includes not only the continuation $s_{f}$ (resp. $s_{h}$ ) of the service performing the receive, but also the activity for sending the response to the customer. This is different from most process calculi and accounts for easily expressing variables shared among parallel activities within the same service instance, which is a feature typically supported in SOC.

The behaviour of the above service is of particular interest when it is included in a scenario with multiple customers (the specifications of customers and searching services are omitted, we just assume that they follow the communication protocol established by the broker specification):

\section{Customer $_{1} \mid$ Customer $_{2} \mid$ Broker' $^{\text {| FlightBooking | HotelBooking }}$}

After a certain number of computational steps have taken place, we can obtain a system configuration where one instance of the broker service is created per each customer, and both instances have sent their requests to the searching services and are waiting for replies. Now, to send the values resulting from the processing of the request of the first customer, the flight searching service has to perform an invoke activity of the form $p_{b r} \bullet o_{f R e s} !\left\langle p_{c 1}, v_{\text {dates }}, v_{\text {dest }}, v_{\text {flight }}\right\rangle$. However, the broker service has two instances waiting for such message along the endpoint $p_{b r} \bullet o_{f R e s}$. In order to deliver the message to the proper instance, i.e. the one serving the request of the first customer, the message correlation mechanism is used. In fact, in SOC, it is up to each single message to provide a form of context that enables services to associate the message with the appropriate instance. This is achieved by embedding values, called correlation data, in the message itself. Pattern-matching is the mechanism used by the Cows's semantics for locating correlation data. In our example, these data are the customer's partner name, the travel dates and the destination, which have instantiated the corresponding variables in the receive activity $p_{b r} \bullet o_{\text {fRes }} ?\left\langle p_{c 1}, v_{\text {dates }}, v_{\text {dest }}, x_{\text {flight }}\right\rangle$ within the broker instance serving Customer $_{1}$. While the receive of the instance serving the first customer is enabled, the one within the other broker instance is not, as it has been instantiated with unmatchable values.

Finally, let us provide further details of the broker specification, in order to add fault and compensation handling activities (highlighted by a grey background): 


$$
\begin{aligned}
& \text { Broker }^{\prime \prime} \triangleq *\left[x_{\text {cust }}, x_{\text {dates }}, x_{\text {dest }}\right] p_{\text {br }} \bullet o_{\text {req }} ?\left\langle x_{\text {cust }}, x_{\text {dates }}, x_{\text {dest }}\right\rangle . \\
& {\left[p, o, x_{\text {flight }}, x_{\text {hotel }}, k\right]} \\
& \left(\left(p_{\text {flight }} \bullet o_{\text {book }} !\left\langle p_{\text {br }}, x_{\text {cust }}, x_{\text {dates }}, x_{\text {dest }}\right\rangle\right.\right. \\
& \mid p_{\text {br }} \bullet o_{\text {fRes }} ?\left\langle x_{\text {cust }}, x_{\text {dates }}, x_{\text {dest }}, x_{\text {flight }}\right\rangle \text {. } \\
& \left(p \bullet o !\langle e n d\rangle \mid s_{f}\right. \\
& \left.\mid\left\{\mid p \bullet o ?\langle\text { comp }\rangle \cdot p_{\text {flight }} \bullet o_{\text {cancel }} !\left\langle x_{\text {cust }}, x_{\text {dates }}, x_{\text {dest }}\right\rangle \mid\right\}\right) \\
& +p_{\text {br }} \bullet o_{\text {fFault }} ?\left\langle x_{\text {cust }}, x_{\text {dates }}, x_{\text {dest }}\right\rangle \text {. } \\
& (\operatorname{kill}(k) \mid\{p \bullet o !\langle\operatorname{com} p\rangle \mid p \bullet o !\langle\text { fault }\rangle\})) \\
& \mid\left(p_{\text {hotel }} \bullet o_{\text {book }} !\left\langle p_{\text {br }}, x_{\text {cust }}, x_{\text {dates }}, x_{\text {dest }}\right\rangle\right. \\
& \mid p_{\text {br }} \bullet o_{\text {hes }} ?\left\langle x_{\text {cust }}, x_{\text {dates }}, x_{\text {dest }}, x_{\text {hotel }}\right\rangle \text {. } \\
& \left(p \bullet o !\langle e n d\rangle \mid s_{h}\right. \\
& \left.\mid\left\{\left|p \bullet o ?\langle\operatorname{comp}\rangle \cdot p_{\text {hotel }} \bullet o_{\text {cancel }} !\left\langle x_{\text {cust }}, x_{\text {dates }}, x_{\text {dest }}\right\rangle\right|\right\}\right) \\
& +p_{b r} \bullet o_{\text {hFault }} ?\left\langle x_{\text {cust }}, x_{\text {dates }}, x_{\text {dest }}\right\rangle \text {. } \\
& (\operatorname{kill}(k) \mid\{p \bullet o !\langle\operatorname{com} p\rangle \mid p \bullet o !\langle\text { fault }\rangle\})) \\
& \mid p \bullet o ?\langle\text { end }\rangle . p \bullet o ?\langle\text { end }\rangle . x_{\text {cust }} \bullet o_{\text {resp }} !\left\langle x_{\text {fight }}, x_{\text {hotel }}\right\rangle \\
& \left.\mid\left\{\mid p \bullet o ?\langle\text { fault }\rangle . x_{\text {cust }} \bullet o_{\text {fault }} !\langle\rangle \mid\right\}\right)
\end{aligned}
$$

Now, when a positive response from a searching service is received, a compensation handler is installed. This consists of an invoke activity on operation $o_{\text {cancel }}$, triggered by a comp signal, devoted to cancel the booking. If a negative response on $o_{f F a u l t}$ (resp. $o_{\text {hFault }}$ ) is received, the normal execution of the service is immediately terminated (by means of the kill activity), the activity compensating the hotel (resp. flight) booking is activated, if installed, and a fault signal is emitted. This last signal triggers the execution of the fault handler, consisting of an invoke activity for notifying the customer that the request booking is failed. Notably, fault and compensation activities are enclosed within protection blocks, in order to protect them from the killing effect of the kill activities.

Discussion. Most of the distinguishing features of Cows find their full application in the final specification of the travel booking scenario. Let us focus on the Broker" service. The replication operator is used to allow the broker service to create multiple instances. In particular, an instance is created for each received customer request. Pattern-matching (on the correlation values replacing variables $x_{c u s t}, x_{d a t e s}$, and $\left.x_{d e s t}\right)$ is then used to associate each 
message from the searching services to the appropriate broker instance. The delimitation operator is used for different purposes: to define the scope of the correlation variables; to make the endpoint $p \bullet o$ private; to share variables $x_{\text {flight }}$ and $x_{\text {hotel }}$ among the parallel terms within the scope of the inner delimitation operator, and to limit the scope of the $\operatorname{kill}(k)$ actions. The protection operator, instead, is used to protect the fault and compensation handlers from the killing effect.

\subsection{Programming environment}

To effectively program SOC applications, Cows, originally conceived as a process calculus, has been extended with high-level features, such as standard control flow constructs (i.e., sequentialisation, assignment, conditional choice, iteration) and a scope activity explicitly defining fault and compensation handlers. The implementation of the resulting orchestration language, called Blite [45], is based on a software tool [46] supporting a rapid and easy development of SOC applications via the translation of service orchestrations written in Blite into executable WS-BPEL programs. More specifically, a Blite program given as input to this tool also includes a declarative part, containing the variable types and the physical service bindings, necessary for generating the corresponding WSDL document and the process deployment descriptor. These files, together with the one containing the WS-BPEL code, are organised in a package that can be deployed and executed in a WS-BPEL engine.

In Fig. 4 we report the relevant $\operatorname{code}^{4}$ of the Blite implementation of the Cows specification, presented in Section 3.4, of the travel booking scenario. Despite the use of a different notation, the invoke (inv) and receive ( $\mathbf{r c v}$ ) primitives of Blite acts similarly to the Cows' ones. To ease the programming task, Blite also provides the high-level features for sequential (seq $; \ldots ;$ _ qes), and parallel (flw $\_|\ldots|{ }_{-}$wlf) composition. These permit avoiding the interactions along the private endpoint $p \bullet o$. The last line of the listing shows a deployment definition, which associates the correlation set $\left\{\mathrm{x} \_\right.$cust, $\mathrm{x} \_$dates, $\mathrm{x} \_$dest $\}$to the broker service. The declarative part of this Blite program, specifying the configuration data necessary to produce the corresponding WS-BPEL program, is omitted.

\footnotetext{
${ }^{4}$ The Blite source code for the complete scenario can be downloaded from https: //bitbucket.org/tiezzi/jlamp_survey_code/src/master/COWS/blite_code/.
} 


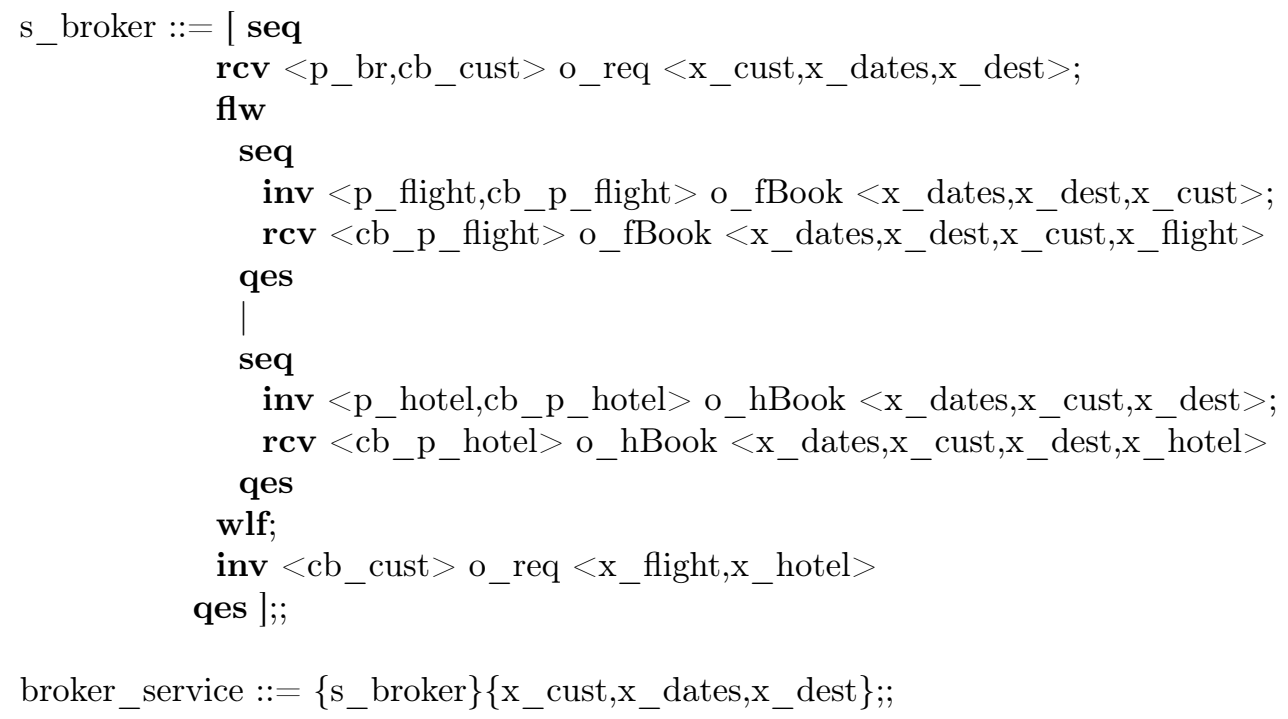

Figure 4: The service Broker' of the travel booking scenario implemented in Blite.

\subsection{Verification techniques}

The main verification techniques devised for Cows specifications are the following: (i) a type system for checking confidentiality properties [47], which uses types to express and enforce policies for regulating the exchange of data among services; (ii) a bisimulation-based observational semantics [48], which permits to check interchangeability of services and conformance against service specifications; (iii) a verification methodology for checking functional properties specific of SOC systems [49].

Concerning the third technique, the properties are described by means of SocL, a logic specifically designed to express in a convenient way distinctive aspects of services, such as, e.g., acceptance of a request, provision of a response, and correlation among service requests and responses. The verification of SocL formulae over Cows specifications is assisted by the on-the-fly model checker CMC. This approach has been used in $[49,50,51]$ to verify some properties of interest of an automotive scenario, an e-Health authentication protocol, and a finance case study, respectively. CMC can also be used as an interpreter for Cows: it takes a Cows term as an input and analyses it syntactically; if the analysis succeeds, the tool allows the user to interactively explore the computations arising from the term.

Using the SocL logic and the CMC tool, we were able to specify and verify 
some properties of the broker service of the travel booking scenario, like:

- The service always gives a response to a request.

that is expressed by the SocL formula

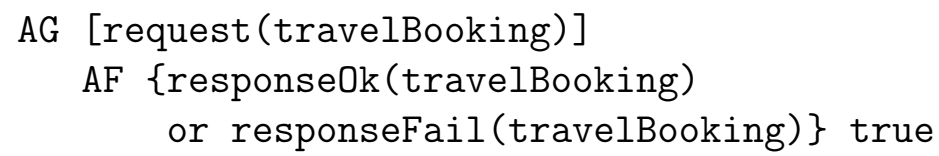

The above property is satisfied by the broker service, as well as the following ones:

- The service is permanently available, i.e. it is always available to accept new requests.

- It is possible to cancel a request after a successful response.

Instead, the following properties are not satisfied:

- The service is sequential, i.e. it will not be available at least until a response is provided.

- The service is reliable, i.e. it always responds positively.

For the latter two properties, CMC can show a counterexample, i.e. a clear and detailed explanation of the negative verification result.

\subsection{Related work}

Cows covers typical aspects of SOC technologies, such as service instances and their interactions, delivery of correlated messages, concurrent activities, multiple start activities, receive conflicts, long-running business transactions. Linguistic variants of Cows have been subsequently introduced to incorporate other aspects, initially not supported by the calculus, such as timed business process activities [52] and dynamic service discovery and negotiation mechanisms [53]. Other variants have also been devised to enable quantitative reasoning on service behaviours [54, 55].

Many other formalisms for SOC have been defined as enrichments of existing process calculi with constructs inspired by those of WS-BPEL. For example, the $\pi$-calculus has been extended in $[56,57,58,59]$ to study a simplified version of the 'scope' construct of WS-BPEL, while CSP has been extended with compensation mechanisms in [60]. Differently from these works, 
when designing Cows the starting point was the technological perspective provided by the OASIS standard WS-BPEL and the related web service technology. Indeed, Cows aims at conveying, in a distilled form, different key notions underlying SOC, to make a direct representation of SOC applications possible.

Another large body of work on modelling of SOC interactions relies on the explicit use of interaction sessions (by exploiting private channels à la $\pi$ calculus). This is the case, e.g., of the SCC [61] and Caspis [62] formalisms. Cows, instead, does not provide an explicit representation of sessions, which anyway can be identified by correlating the related messages by their content. This is the approach fostered by the SOC technology (in particular by WS$\mathrm{BPEL}$ ), as it is more robust and fits better with the loosely coupled nature of SOC than that based on explicit session references.

The formalism closest to Cows is, perhaps, SOCK [63], as it also relies on a form of correlation-based communication, but Cows is more amenable to formal reasoning, as it has a much simpler operational semantics. SOCK is a three-layered calculus, which decomposes a SOC specification into three parts: the behaviour (process description), the declaration (concerning execution modalities, e.g. concurrent vs. sequential) and the composition (consisting of the parallel composition of service engines). Cows represents a more foundational formalism than SOCK, in that it does not explicitly consider the declaration layer. The interested reader is referred to [7] for a description of the way services' execution modalities can be rendered in Cows.

For other references on service-oriented computing and relation with Cows we refer the interested reader to [7, 64].

More recently, a new architectural style for structuring applications as a collection of services is emerging. It is called microservices and shares with SOC many design principles, e.g. complex distributed applications structured in terms of loosely coupled, independent and interoperable components. However, microservices are more lightweight than web services from the technological point of view, e.g. they interact asynchronously by directly using the HTTP protocol. In particular, microservices do not exploit the OASIS standard WS-BPEL for their orchestration, which is instead at the basis of the design of Cows. Therefore, in the first instance, Cows does not seem suitable for directly modelling microservices, but we leave a more thorough investigation of this issue for future work. 


\section{SCEL: Software Component Ensemble Language}

Developing massively distributed and highly dynamic computing systems which control and interact with the physical world is a major challenge in todays software engineering. Difficulties arise from the open-ended and dynamic nature of large-scale systems, the unpredictable external environment, the limited if not impossible human intervention, and the need of ensembles of components to interact and collaborate for achieving specific goals, while hiding complexity to end-users. A possible answer to the problems posed by such systems is to make them self-aware and context-aware, and able to self-adapt and to self-configure. These and other self-management capabilities, like self-healing, self-optimisation, and self-protection, characterise autonomic computing [4] systems. In order to achieve these goals, it is needed that these systems continuously monitor their progress and the environment they are working in, to determine the actions to perform and the components to install for better dealing with the current status of affairs.

Specific support to programming these systems is provided by SCEL (Software Component Ensemble Language) [8, 65] a formal language equipped with a set of linguistic abstractions for specifying the behaviour of components, the interaction among them, and the dynamic formation of their ensembles. In SCEL, components are computational entities that have associated knowledge repositories and specific behavioural policies. They also have an interface used to expose their characterising features (attributes). Ensembles, in turn, are aggregations of interacting components that are determined at run-time by means of predicates over components' attributes.

SCEL components acquire information about their status (self-awareness) and about their environment (context-awareness) from knowledge repositories. Moreover, by exploiting awareness and the capability of adding processes to knowledge repositories and of dynamically activating processes and components, they can guarantee self-adaptation and self-configuration, initiate self-healing actions in presence of faults, and activate self-optimisation strategies. Finally, by using suitable policy languages, components can attain data integrity and self-protection against, e.g., unauthorised accesses or denial-of-service attacks.

A system of collaborative SCEL components can thus monitor its state and its components, as well as the execution context, and identify relevant changes that may affect the achievement of its goals or the fulfilment of its requirements. The system can then plan reconfigurations to meet the new 
functional or non-functional requirements, execute them, and monitor that its goals are achieved, possibly without any interruption. Attributes are key to support this autonomic behaviour; they are used to expose distinguishing features of SCEL components and to indicate occurrence of specific events. The set of all exposed attributes constitutes the common knowledge that is updated during computations and is used to build patterns of communication to enable SCEL components to dynamically organise themselves into ensembles.

A careful choice and use of (sets of) attributes permit to express autonomic behaviours in SCEL in a natural way as done, e.g., in [66] for modelling component- and ensemble-level adaptation patterns, and in [67] for offering self-expression, i.e. the ability of changing at run-time the coordination pattern used in an ensemble. SCEL has also been used for effectively modelling autonomic systems from different application scenarios such as, e.g., swarm robotics [68, 8], cooperative e-vehicles [69], service provision and cloud-computing $[70,71,72]$.

\subsection{Syntax}

SCEL syntax is reported in Table 5. Five pairwise disjoint sets are used: Names (ranged over by $n, n^{\prime}, \ldots$ ), Predicate names (ranged over by $p$, $p^{\prime} \ldots$ ), Variables for names (ranged over by $x, x^{\prime}, \ldots$ ), Variables for processes (ranged over by $X, Y, \ldots$ ), and Parameterised process identifiers (ranged over by $A, B \ldots$ ). The distinguished variable self is used to denote the name of the component using it.

Systems are obtained by aggregating COMPONENTS which, in turn, are obtained by aggregating KNOWLEDGE and PROCESSES, according to some Policies. Processes specify the flow of the ACTIONs that can be performed and use the same composition operators as in KLAIM. ACTIONS can have a TARGET to determine the components, in addition to the subject one, that are involved in that action.

SCEL is parametric with respect to some syntactic categories, namely Policies, Knowledge, Templates and Items (with the last two determining the part of KNOWLEDGE to be retrieved/removed or added, respectively). This choice permits integrating different approaches to policy specification and knowledge handling within SCEL, like, e.g., the access control policies of [71] and the constraint stores of [73].

A simple, yet expressive, instance of SCEL, named ScElight, has been introduced in [74] where policies are absent (equivalently, where any process 


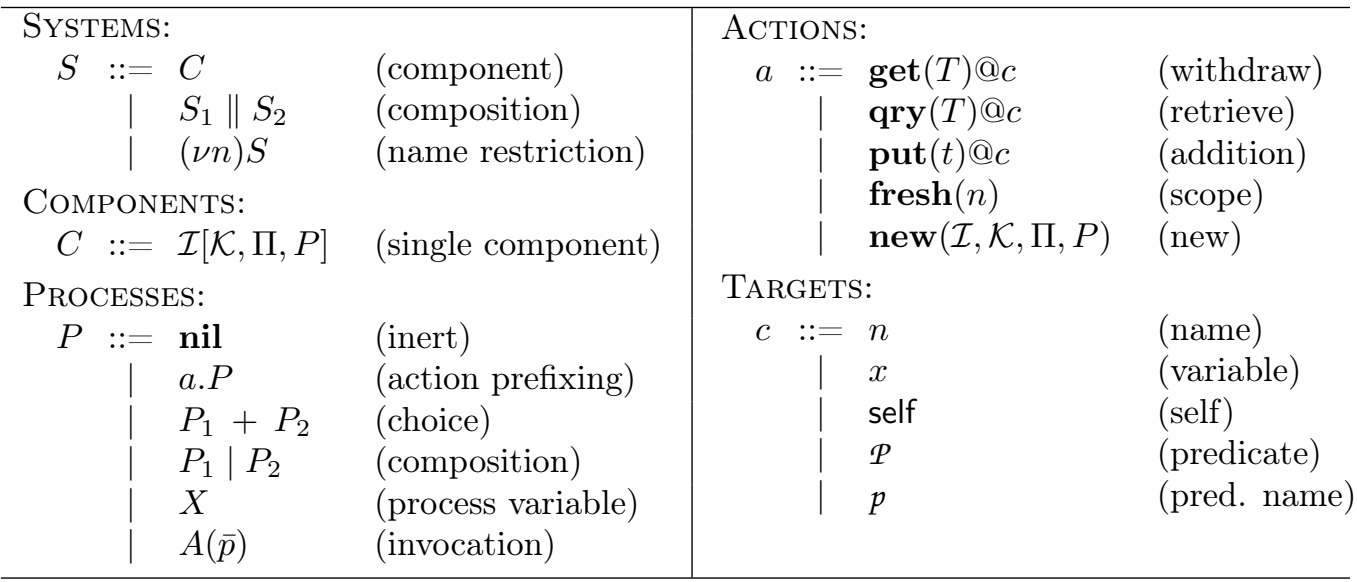

Table 5: Scel syntax (Policies $\Pi$, Knowledge $\mathcal{K}$, Templates $T$, and Items $t$ are parameters of the language)

action is authorised) and knowledge repositories are implemented as tuple spaces á la Klaim. A full-fledged instance, named Pscel (Policed SCEL), has been introduced in [65]. In PSCEL, knowledge repositories are again implemented as KLAim tuple spaces, while policies are expressed by means of a simplified version of FACPL (Formal Access Control Policy Language, [75]), a language for defining access control, resource usage and adaptation policies.

\subsection{Informal semantics}

A SCEL SYSTEM in an aggregation of COMPONENTS built by means of the composition operator _ $\|_{-}$, which is both commutative and associative. Within a system, it is also possible to restrict to a subsystem the scope of a name, say $n$, by using the name restriction operator $(\nu n)$. Thus, in a system of the form $S_{1} \|(\nu n) S_{2}$, the effect of the operator is to make name $n$ invisible from $S_{1}$.

A Scel Component $\mathcal{I}[\mathcal{K}, \Pi, P]$ consists of:

- An interface $\mathcal{I}$ publishing and making available information about the component itself in the form of attributes, i.e. names acting as references to information stored in the component's knowledge repository. Among them, attribute $i d$ is mandatory and is bound to the name of the component. 
- A knowledge repository $\mathcal{K}$ managing both application data and awareness data, equipped with specific handling mechanisms. Application data are used for determining the progress of components' computations, while awareness data provide information about the environment in which the components are running or about the status of the component itself.

- A set of policies $\Pi$ regulating the interaction between the different processes of the component and the interaction with other components.

- A process $P$, together with a set of process definitions that can be dynamically activated.

Processes are the active computational units. Each process is obtained by composing subprocesses or the inert process nil via action prefixing (a.P), nondeterministic choice $\left(P_{1}+P_{2}\right)$, controlled composition $\left(P_{1} \mid P_{2}\right)$, process variable $(X)$, and parameterised process invocation $(A(\bar{p}))$. The semantics of the construct $P_{1} \mid P_{2}$ is another parameter of SCEL. It can be instantiated to capture various forms of parallel composition commonly used in process calculi. For example, in PSCEL, it corresponds to the interleaved execution of the two involved processes. Communication can be higher-order, as in Klaim. We assume that $A$ ranges over a set of parameterised process identifiers that are used in (possibly recursive) process definitions. It is also assumed that each process identifier $A$ has a single definition of the form $A(\bar{f}) \triangleq P$. Lists of actual and formal parameters are denoted by $\bar{p}$ and $\bar{f}$, respectively.

Processes can perform five different kinds of ACTIONS. Actions get $(T) @ c$, qry $(T) @_{c}$ and $\operatorname{put}(t) @_{c}$ are used to manage shared knowledge repositories by withdrawing/retrieving/adding information items from/to the knowledge repositories identified by the target $c$. These actions exploit templates $T$ as patterns to select knowledge items $t$ from the repositories. They depend on the chosen kind of knowledge repository (a parameter of SCEL, as pointed out earlier) and are implemented by invoking the provided knowledge handlers. Action fresh $(n)$ introduces a scope restriction for the name $n$ to guarantee that this name is fresh, i.e., different from any other previously used name. Action new $(\mathcal{I}, \mathcal{K}, \Pi, P)$ creates a new component $\mathcal{I}[\mathcal{K}, \Pi, P]$.

Actions get/qry may block the executing process to wait for the expected item, in case it is not (yet) available in the knowledge repository. The two 
actions differ for the fact that get removes the found item from the knowledge repository while qry leaves the target repository unchanged. Actions put, fresh and new are instead immediately executed (provided that their execution is allowed by the policies in force).

To indicate the target $c$ of an action, in addition to names and variables for names, the process performing the action can also use the distinguished variable self, to refer to the name of the hosting component, or a predicate $\mathcal{P}$ (or the name $p$ of a predicate), to specify the ensemble of all those components with which the process wants to interact. Predicates are boolean-valued expressions obtained by logically combining relations involving attributes. Thus, e.g., actions put $(t) @ n$ and put $(t) @ \mathcal{P}$ give rise to two different primitive forms of communication: the former is a point-to-point communication, while the latter is a sort of group-oriented communication.

It is worth noticing that the group-oriented variant of action put is used to insert a knowledge item in the repositories of all components belonging to the ensemble identified by the target predicate. Differently, the grouporiented variants of actions get and qry withdraw and retrieve, respectively, an item from a single component non-deterministically selected among those satisfying the target predicate.

\subsection{A taste of the operational semantics}

The semantics of SCEL is defined by means of a few (Labeled) Transition Systems. In this section we introduce the inference rules, shown in Table 6, of the labeled transition relation defining the behaviour of all the variants of the action put: that for point-to-point communication, which can be either local (rule (lput)) or remote (rule (ptpput)), and that for group-oriented communication (rules (grput) and (engrput)). The semantics of the other actions is defined similarly. We refer the interested reader to $[8,65]$ for a full account of SCEL operational semantics.

The execution of actions put by a component, which is indicated by the transition label $\mathcal{I}: t \triangleright \gamma$ generated by rule (put), requires an appropriate synchronisation with one or more target components allowing addition of the argument item to their local repository. These components perform a fictitious authorisation action, which is indicated by the transition label $\mathcal{I}: t \bar{\triangleright} \mathcal{J}^{\prime}$ generated by rule (accput). Eventually, the simultaneous execution of these complementary actions gives rise to a computation step, which is denoted by a $\tau$-labelled transition generated, e.g., by rules (lput) and (ptpput). 


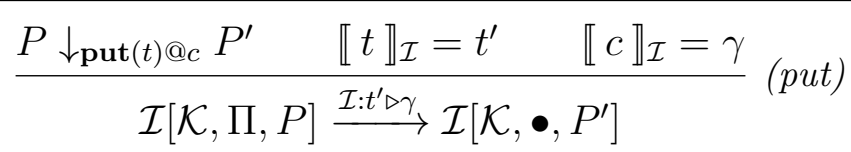

$$
\begin{aligned}
& \frac{\Pi \vdash \mathcal{I}: t \overline{\mathcal{J}}, \Pi^{\prime} \quad \mathcal{J}^{\prime}=\mathcal{J}\left[\Pi^{\prime} / \mathcal{J} . \pi\right] \quad \mathcal{K}^{\prime}=\mathcal{K} \oplus t}{\mathcal{J}[\mathcal{K}, \Pi, P] \stackrel{\mathcal{I}: t \overline{\mathcal{J}^{\prime}}}{\longrightarrow} \mathcal{J}\left[\mathcal{K}^{\prime}, \Pi^{\prime}, P\right]} \text { (accput) } \\
& \frac{C \stackrel{\mathcal{I}: t \triangleright n}{\longrightarrow} C^{\prime} \quad n=\mathcal{I} \text {.id } \quad C^{\prime}[\mathcal{I} . \pi / \bullet] \stackrel{\mathcal{I}: t \bar{\perp}}{\longrightarrow} C^{\prime \prime}}{C \stackrel{\tau}{\rightarrow} C^{\prime \prime}} \text { (lput) }
\end{aligned}
$$

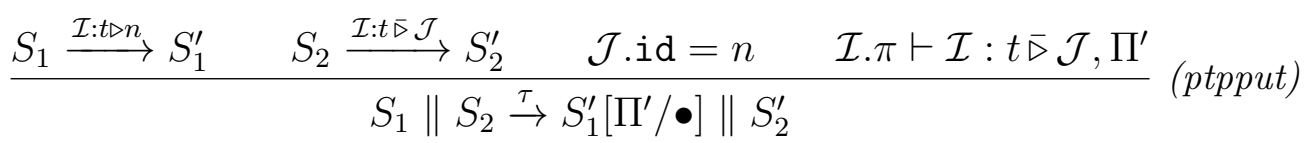

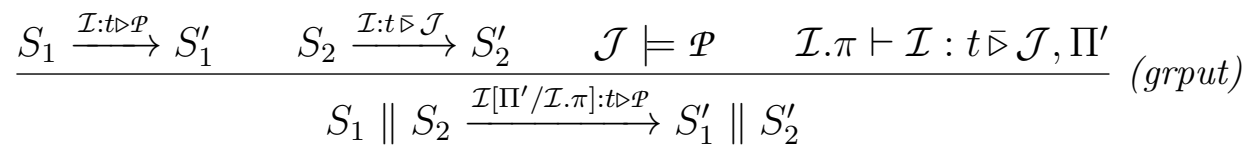

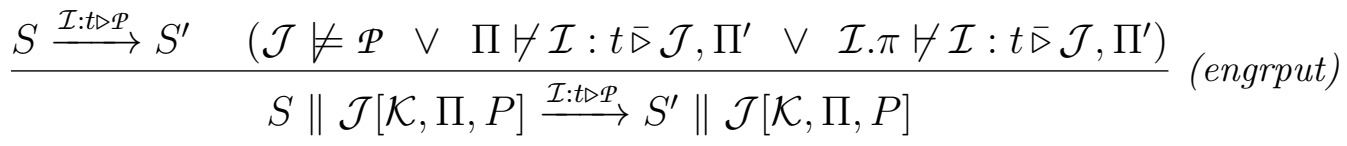

Table 6: Inference rules for the action put ( $\mathcal{I}$ and $\mathcal{J}$ range over interfaces)

More specifically, rule $(p u t)^{5}$ indicates the intention of component $\mathcal{I}$ to perform an action put corresponding to a commitment of the process $P$ running locally which becomes $P^{\prime}$ after execution of the action. Function $\llbracket \cdot \rrbracket_{\mathcal{I}}$ denotes the evaluation of terms with respect to the interface $\mathcal{I}$, causing the replacement of the attributes occurring therein by the corresponding information and the replacement of self by the component name (i.e. $\mathcal{I}$.id). Therefore, $t^{\prime}$ is an evaluated item and $\gamma$ is either the name $n$ of a single component or a predicate $\mathcal{P}$ indicating a set of components. Notably, the policy in force at the component performing the action might change. Indeed, after the transition, the component contains a placeholder $\bullet$ in place of the policy; it will be replaced by an actual (possibly new) policy when the target

\footnotetext{
${ }^{5}$ Actually, this rule is an instantiation and a simplification of the original rule ( $p r$ sys) $[8,65]$ for only taking into account actions put, as done in the present context.
} 
$\gamma$ of the action is determined (see the rules (lput) and (ptpput)).

Rule (accput) indicates that the policy $\Pi$ of component $\mathcal{J}$ allows component $\mathcal{I}$ to add the item $t$ to $\mathcal{J}$ 's repository $\mathcal{K}$ thus getting the repository $\mathcal{K}^{\prime}$ (this is denoted by $\mathcal{K} \oplus t=\mathcal{K}^{\prime}$ ). This control is done by the so called authorisation predicate ${ }^{6}$ which is the first premise of the rule. An effect of this transition is the possible update of policy $\Pi$ which becomes $\Pi^{\prime}$, both in the component after the transition and in the generated label (in fact, $\mathcal{J}^{\prime}$ is $\mathcal{J}$ where $\Pi^{\prime}$ replaces the local policy, denoted by $\left.\mathcal{J} . \pi\right)$.

In case of point-to-point communication, action put adds an item either to the local repository (rule (lput)) or to a remote repository (rule (ptput)). Anyhow, this transition corresponds to a computation step. Notably, the transition labelled by $\mathcal{I}: t \bar{D} \mathcal{I}$ in the premise of (lput) can only be produced by rule (accput); it thus ensures that the component $\mathcal{I}$ authorises the local addition of the item $t$ and that the component's knowledge and policy are updated accordingly. When the target of the action denotes a specific remote repository (ptput), the action is only allowed if $n$ is the name of the component $\mathcal{J}$ simultaneously willing to accept addition of the item to its local repository and if the request to perform the action at $\mathcal{J}$ is authorised by the local policy (this is checked by the authorisation predicate occurring as last premise). Notably, if there are multiple components with the same name $n$ willing to accept the item, one of them is non-deterministically chosen.

In case of group-oriented communication, in a single transition action put adds an item to the repository of each component satisfying its target predicate $\mathcal{P}$ and willing to accept it. Indeed, rule (grput) permits the execution of a put for group-oriented communication when there is a parallel component, say $\mathcal{J}$, satisfying the target of the action and whose policy authorises this remote access. Relation $\mathcal{J} \models \mathcal{P}$ states that predicate $\mathcal{P}$ holds true when evaluated after replacement of the attributes occurring therein with the information to which these attributes are bound within the interface $\mathcal{J}$. The exact definition of such relation depends on the used predicates. Of course, the action put must be authorised to use $\mathcal{J}$ as a target also by the policy in force at the component performing the action (the policy is updated after each evaluation of the authorisation predicate). Notably, the label of the inferred transition is yet that of a put for group-oriented communication,

\footnotetext{
${ }^{6}$ The actual definition of this predicate depends on the policy language, which is one of the parameters of SCEL.
} 


\begin{tabular}{lll}
\hline KNOWLEDGE: & Items: & Templates: \\
$\mathcal{K}::=\emptyset|\langle t\rangle| \mathcal{K}_{1} \| \mathcal{K}_{2}$ & $t::=e|c| P \mid t_{1}, t_{2}$ & $T::=e|c| ? x|? X| T_{1}, T_{2}$ \\
\hline
\end{tabular}

Table 7: Tuple space syntax ( $e$ is an ExPREssion)

thus further authorisation actions performed by other parallel components satisfying the target of the action can be simultaneously executed. In other words, the rule implies that multiple components can be delivered the same item in a single transition. Instead, rule (engrput) means that the capability of a component to perform a put for group-oriented communication is not affected by those other components not satisfying predicate $\mathcal{P}$, i.e. not belonging to the ensemble, or not authorised by the executing component or not authorising the action. Thus, when there is a component performing a put for group-oriented communication, by repeatedly applying rules (grput) and (engrput), in one way or another all system components get involved in the transition, which can then be turned into a computation step (this is done by a rule of a different transition system not shown here).

\subsection{A travel booking scenario}

Our specification of the scenario is written in PSCEL, which instantiates knowledge repositories, items and templates of the SCEL syntax as shown in Table 7. Notably, knowledge ITEMS are tuples, i.e. sequences of values, while TEMPLATES are sequences of values and formal fields binding variables. Values can be either targets $c$, or processes $P$ or, more generally, the result of the evaluation of an expression $e$. We assume that expressions may contain attribute names, boolean, integer, float and string values and variables, and the standard operators over them. A KLAIM-like pattern-matching mechanism is used to withdraw a tuple from a tuple space when a given template is specified.

Regarding PSCEL policies ${ }^{7}$, for the purposes of this example it is sufficient to know that they are hierarchically structured lists of elements containing controls on the value of attributes that should be provided by access requests generated when executing process actions. Together with permit or deny decisions, policies specify the conditions for their applicability, the combination algorithms to be used in their evaluation and the obligations for the enforce-

\footnotetext{
${ }^{7}$ We refer the interested reader to [65] for the presentation of the syntax of the policy language and of the operational semantics of PSCEL.
} 


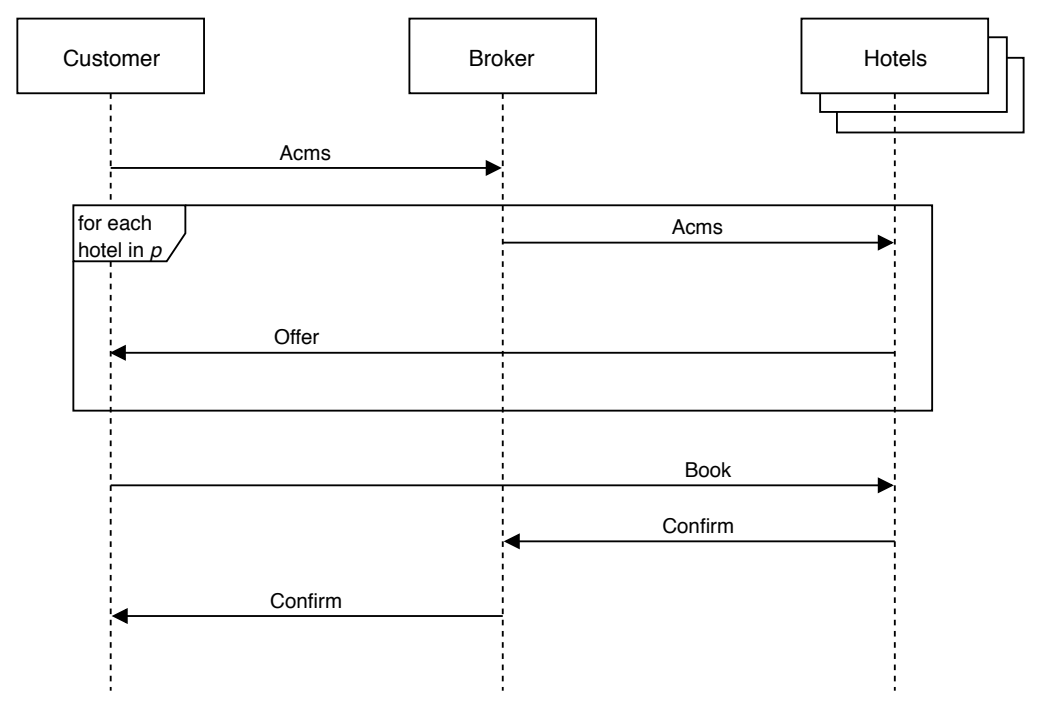

Figure 5: Travel Booking Scenario in ScEL: Sequence Diagram.

ment process. Due to space limitations, we only explicitly show the policy of the customers, being those of the other components similar.

The scenario ${ }^{8}$ is modelled by the following system $S$ including two customer components $\mathcal{I}_{c}\left[\mathcal{K}_{c}, \Pi_{c}, P_{c}\right]$ and $\mathcal{I}_{d}\left[\mathcal{K}_{d}, \Pi_{d}, P_{d}\right]$, a broker component $\mathcal{I}_{b}\left[\mathcal{K}_{b}, \Pi_{b}, P_{b}\right]$, and $n$ hotel components $\mathcal{I}_{h_{i}}\left[\mathcal{K}_{h_{i}}, \Pi_{h_{i}}, P_{h_{i}}\right]$ :

$$
\begin{aligned}
S \triangleq & \mathcal{I}_{c}\left[\mathcal{K}_{c}, \Pi_{c}, P_{c}\right]\left\|\mathcal{I}_{d}\left[\mathcal{K}_{d}, \Pi_{d}, P_{d}\right]\right\| \mathcal{I}_{b}\left[\mathcal{K}_{b}, \Pi_{b}, P_{b}\right] \| \\
& \mathcal{I}_{h_{1}}\left[\mathcal{K}_{h_{1}}, \Pi_{h_{1}}, P_{h_{1}}\right]\|\cdots\| \mathcal{I}_{h_{n}}\left[\mathcal{K}_{h_{n}}, \Pi_{h_{n}}, P_{h_{n}}\right] .
\end{aligned}
$$

A specific UML activity diagram for $S$ is reported in Fig. 5. Here, customer and hotel components can directly interact; this simplifies the model as it avoids the broker component to select room offers and make reservations on behalf of the customers. The figure also shows that the broker component forwards customers' requests to all those hotel components satisfying a given predicate $p$, which can then send offers directly to the requesting customer. The next room booking message is instead only directed to one of the hotels, which finally sends a confirmation to the customer through the broker component. In the rest of this section, we describe the different components of the system.

\footnotetext{
${ }^{8}$ We focus on hotel booking as flight booking is really similar.
} 
Customer. The following process $P_{c}$ runs at the customer component $\mathcal{I}_{c}$ :

$$
\begin{aligned}
& P_{c} \triangleq \operatorname{fresh}(k e y) \text {. } \\
& \operatorname{put}(\text { "Acms", loc, day, rating, price, self, key }) @\left(\mathcal{I}_{b}\right. \text {.id). } \\
& P_{c}^{\prime}(\text { day }, \text { key }) \\
& P_{c}^{\prime}(d, k) \triangleq \operatorname{get}(\text { "Offer", } d, ? f, ? p, ? h, k) @ \text { self. } \\
& \text { ( put("Book", } d, f, k) @ h \text {.get("Confirm", } d, ? X, k) @ \text { self. } P_{c}^{\prime \prime}(X) \\
& \left.+P_{c}^{\prime}(d, k)\right)
\end{aligned}
$$

This process firstly creates a name, denoted by key, which is guaranteed to be unique in the whole system. This name is a reference key for identifying the inquiry, thus permitting to distinguish possibly simultaneous inquiries generated by the two customers and to correlate responses to the corresponding inquiry. Then, the process sends a room reservation inquiry to the broker component by exploiting remote point-to-point communication. In addition to the string "Acms", the inquiry specifies: destination locality, day of stay, minimum star-rating, and the maximum room-price that the customer is willing to pay. The inquiry also contains the customer's identity and the name identifying the request. The continuation process $P_{c}^{\prime}(d, k)$ elaborates the received room offers. By exploiting the pattern-matching mechanism, the process firstly withdraws from the local repository an offer (if any) corresponding to the inquiry done. The offer specifies features and price of the room, and the hotel's identity. Then, non-deterministically, either it sends a message to the hotel for reserving the room and waits for a confirmation, or (discards the offer and) continues recursively by withdrawing another offer.

In a real case study, the actual hotel would be selected after comparing price and features of the room and the related services. Here, for the sake of simplicity, we only model the selection as a non-deterministic choice. Also, we do not consider the possibility that a customer gets stuck due to lack of offers and we do not model removal of offers corresponding to finalised reservations.

In case room reservation is confirmed, by means of higher-order communication, the customer receives a process that is bound to variable $X$ and can be, later on, sent for execution by $P_{c}^{\prime \prime}$, e.g. to take advantage of some complimentary services provided by the broker. These services, together with special rates agreed between the broker and the contacted hotels, represent the advantage for the customer in booking hotels through the broker rather than directly. 
The policy $\Pi_{c}$ in force at the customer $\mathcal{I}_{c}$ is defined as follows:

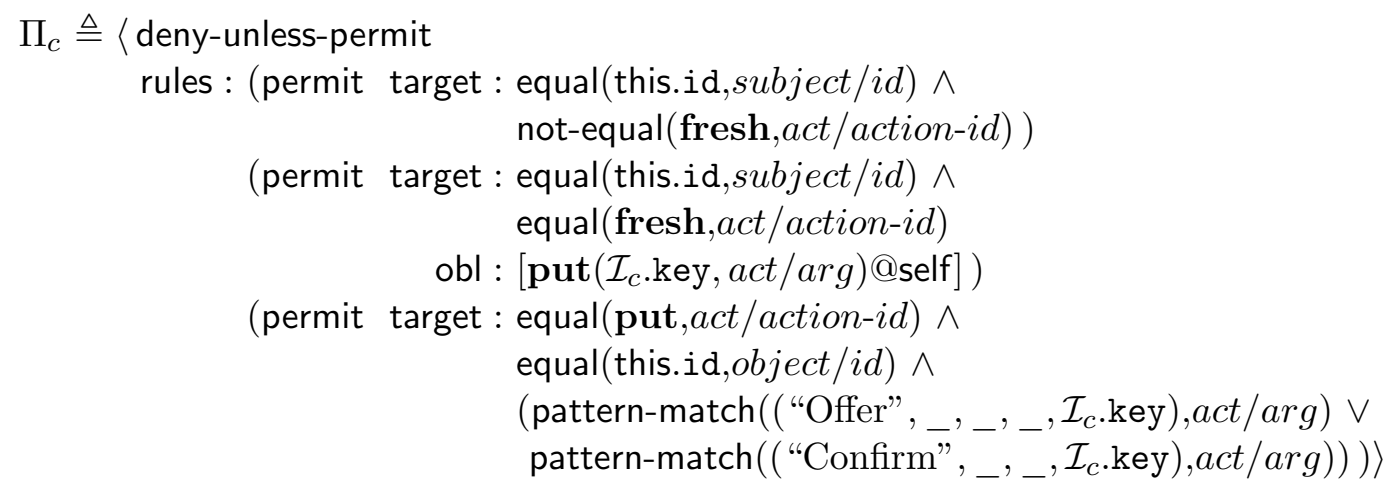

The policy consists of three rules combined through the algorithm named deny-unless-permit whose effect is to disallow any action that is not explicitly allowed by the rules. The first rule allows the local process (that is, $P_{c}$ ) to perform any action different from a fresh. In fact, the keyword this denotes the component where the policy is in force (i.e. the customer component), while the name subject identifies the component performing the action which has to be authorised. The target of the rule requires these two components to coincide. The second rule allows the local process to perform an action fresh, but with the obligation of immediately performing a so called obligation action for setting the component attribute key to the argument of fresh (we assume that at the outset no value is bound to key and that components attributes are managed by using the same mechanisms used for data). This means that, in general, policy evaluation can affect the system by adding further behaviours, other than simply (dis-)allowing some behaviours. The last rule allows other components to perform actions put having the local component as a target (i.e. object) provided that the argument of the action is a tuple with a very specific structure, where the last field is the name purposely created to identify the specific inquiry. This check on the tuples aims at protecting the integrity of the repository; it ensures that the tuples added to the repository have a specific structure, with their last field containing the value of the attribute key. Indeed, the check succeed only after a value is bound to key, that is after the local process has performed the action fresh that triggers the obligation action put assigning a value to key.

It is worth noticing that, since SCEL features asynchronous communication, customers' inquiries are directly placed in the broker' repository, as well as hotels' offers are placed in the customers' repository, without requiring any synchronisation with other process actions. However, components 
can use policies to authorise such actions or not and, possibly, to oblige the executing process to perform some actions, like the one in the previous policy about setting the value of the attribute key.

Broker. The following process $P_{b}$ runs at the broker component $\mathcal{I}_{b}$ :

$$
\begin{aligned}
& P_{b} \triangleq \operatorname{get}(" A c m s ", ? l, ? d, ? r, ? p, ? c, ? k) @ \text { self. } \\
& \left(P_{b} \mid \operatorname{put}(" \mathrm{Acms} ", d, c, k) @ p \cdot P_{b}^{\prime}(l, d, c, k)\right) \\
& P_{b}^{\prime}(l, d, c, k) \triangleq \operatorname{get}(" C o n f i r m ", d, ? f, ? h, k) @ \text { self. } \\
& \text { put("Confirm", } d, Q(l, d, f, h), k) @ c . n i l
\end{aligned}
$$

where the predicate name $p$ is defined as

$$
\begin{aligned}
p \triangleq & \text { serviceType }=\text { "hotel" } \wedge \text { locality }=l \wedge \\
& \text { starRating } \geq r \wedge \text { roomPrice } \leq p
\end{aligned}
$$

The process $P_{b}$ is triggered by a customer inquiry withdrawn from the local repository. Whenever this happens, the process continues by spawning, for parallel execution, a copy of itself, to serve other inquiries, and by making a group-oriented put using the data of the customer's inquiry, partly in the target, to filter the interacting partners, and in part in the forwarded inquiry itself. This means that there is no predefined list of hotels to interact with. Rather, a combination of predicates on the attributes named serviceType, locality, starRating, and roomPrice is used at run-time to identify the ensemble made of all hotels which are relevant to deal with the given customer inquiry. Thus, by taking advantage of group-oriented communication, the broker is able to dynamically identify an ensemble of hotel components that can potentially provide the service requested by the customer. All the hotel components meeting these requirements get an excerpt of the original customer inquiry, specifying the day of stay, the customer's identity, and the unique inquiry's reference key. The continuation process $P_{b}^{\prime}$ waits for a confirmation of room reservation, including the features of the room, from a hotel after which it forwards the message to the customer along with a process $Q$ enabling the customer to use some complimentary services provided by the broker and depending on the destination, the day of stay, the paid price, the chosen hotel, etc.

Hotel. The following process $P_{h_{i}}$ runs at the hotel component $\mathcal{I}_{h_{i}}$ : 


$$
\begin{aligned}
& P_{h_{i}} \triangleq \operatorname{get}(\text { "Acms", ?d,?c,?k)@self. } \\
& \left(P_{h_{i}}\left|P_{h_{i}}^{o}(d, c, k)\right| P_{h_{i}}^{b}(d, k)\right) \\
& \left.P_{h_{i}}^{o}(d, c, k) \triangleq \mathbf{q r y} \text { ("Room", } d, ? f\right) @ \text { self. } \\
& \text { put("Offer", } d, f, p\left(f, \mathcal{I}_{b} \text {.id), self, } k\right) @ c . \text { nil } \\
& \left.P_{h_{i}}^{b}(d, k) \triangleq \operatorname{get}(\text { "Book", } d, ? f, k) @ \text { self.get("Room", } d, f\right) @ \text { self. } \\
& \operatorname{put}(\text { "Confirm", } d, f \text {, self, } k) @\left(\mathcal{I}_{b}\right. \text {.id).nil }
\end{aligned}
$$

The process $P_{h_{i}}$, when triggered by a room reservation inquiry withdrawn from the local repository, spawns for parallel execution a copy of itself to serve other, possibly simultaneous, inquiries, elaborates the specific inquiry, and manages the room reservation request. The elaboration of the specific inquiry is done by the process $P_{h_{i}}^{o}$ which retrieves from the local repository information about room availability for the day of stay and hotel features, and sends back to the customer offers including room price. This price is calculated by function $p()$ that, on the basis of room features and broker identity, can return special rates depending on the specific agreements between the hotel and the broker. The process $P_{h_{i}}^{b}$, when triggered by a room reservation request, removes the corresponding room from the availability list for the day of stay, and sends a confirmation to the broker.

Discussion. The specification $S$ of the scenario takes advantage both of SCEL general features and specific ones of the used dialect. Among the former, attributes and predicates over them are used to implement group-oriented communication, to enable the broker component to dynamically select an ensemble of hotels that could provide the service requested by customer components. Moreover, dynamic creation of unique names is used to unambiguously identify customer requests and all correlated messages subsequently exchanged, while process variables are used for exchanging and activating the processes that model the complimentary services provided by the broker. Among the features of the used dialect, KLAIM-like pattern-matching is exploited to selectively access items in the knowledge repositories, while policies are used both to disallow behaviours which could undermine the integrity of the repositories and to generate new behaviours through obligation actions. In particular, obligations have been used for setting some attributes when specific events occur (in this case, creation of fresh unique names). 


\subsection{Programming environment}

SCEL systems can be executed and simulated in jRESP ${ }^{9}$ (Java Runtime Environment for SCEL Programs), which offers specific software tools to develop and support SCEL systems. In particular, jRESP provides an API that permits enriching Java programs with the SCEL's linguistic constructs. The API is instrumental to assist programmers in the implementation of autonomic systems, which thus turns out to be definitely simpler than using "pure" Java. Moreover, jRESP provides a set of classes enabling execution of virtual components on top of a simulation environment that can control component interactions and collect relevant simulation data.

In Fig. 6 we report a significant fragment of $\operatorname{code}^{10}$ of the jRESP implementation of the SCEL specification of the travel booking scenario, presented in Section 4.4. The Java classes of the broker process reported here show how programmers can directly use SCEL communication primitives in Java, thus resulting in a very compact code. A SCEL process is rendered in jRESP as an instance of class Agent, which specifies the agent behaviour within the method doRun(). In the case of the broker, this method first performs a get action to retrieve an Acms tuple, and then spawns another instance of the broker agent in the broker node, to immediately serve other possible customer requests. The method get() takes as parameters an instance of class Template and a target (in this case it is the local component referred by Self.SELF), and returns a matching tuple. In the subsequent put action, instead, the target is an ensemble (instance of class Group), defined by a predicate (instance of class GroupPredicate). Finally, in the last put action the target is the customer address, dynamically retrieved from the Acms tuple initially received.

\subsection{Verification techniques}

Qualitative properties of SCELIGHT specifications have been verified through the Spin model checker [76]. The verification relies on a translation from Scelight into Promela, the input language of Spin. This approach has been used in [74] to verify properties of interest for a service provision scenario, like absence of deadlock, server overload and responsiveness, and in [65] to verify similar properties for a swarm robotics scenario.

\footnotetext{
${ }^{9}$ jRESP website: http://jresp. sourceforge.net/.

${ }^{10}$ The jRESP source code for the complete scenario can be downloaded from https: //bitbucket.org/tiezzi/jlamp_survey_code/src/master/SCEL/.
} 


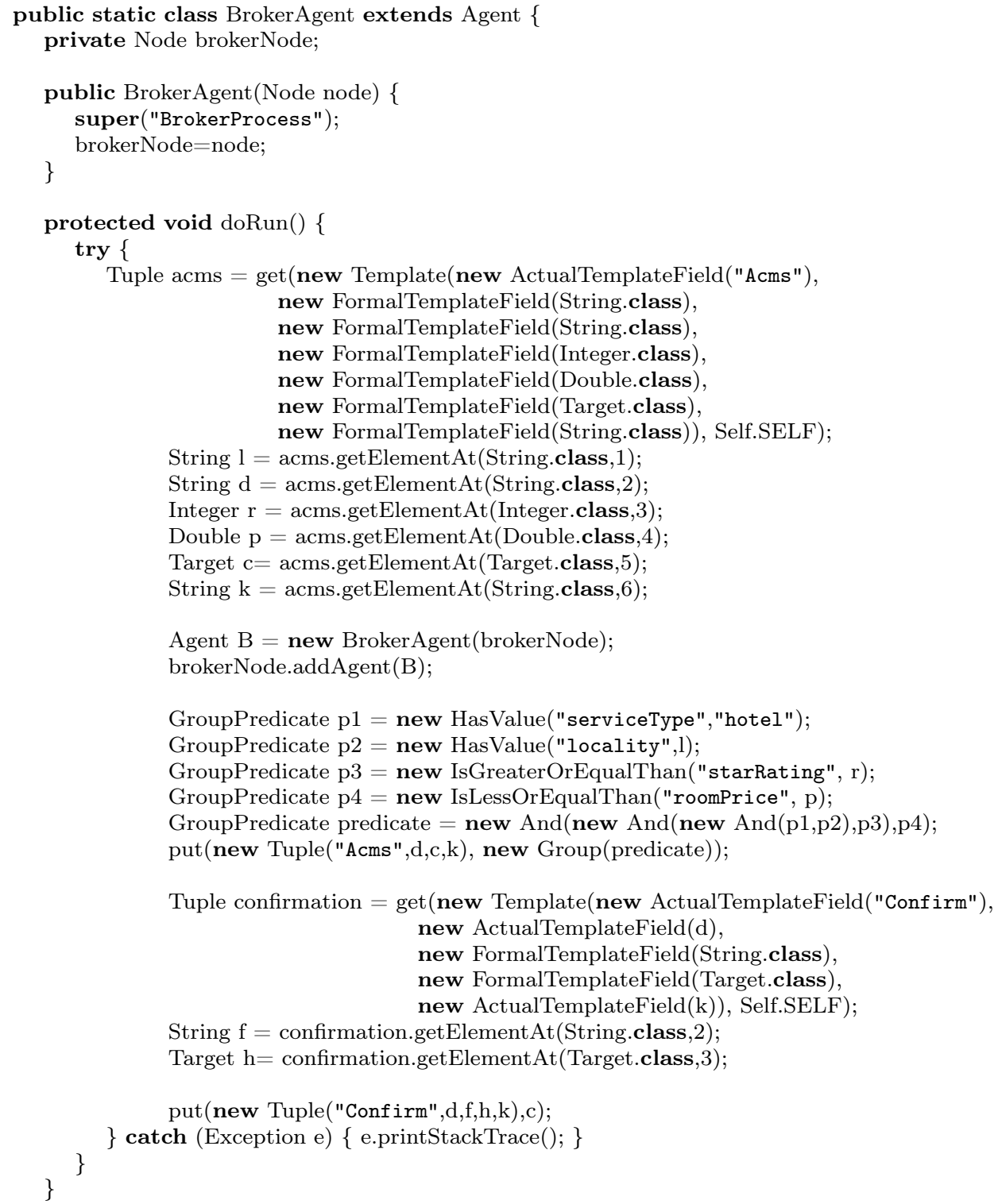

Figure 6: The process $P_{b}$ of the travel booking scenario implemented in jRESP.

SCEL's operational semantics has also been implemented by using the Maude framework [77]. The outcome, named MissCEL (Maude Interpreter and Simulator for SCEL), focuses on SCELIGHT and exploits the rich Maude toolset to perform, among other things, qualitative analysis via Maude's 


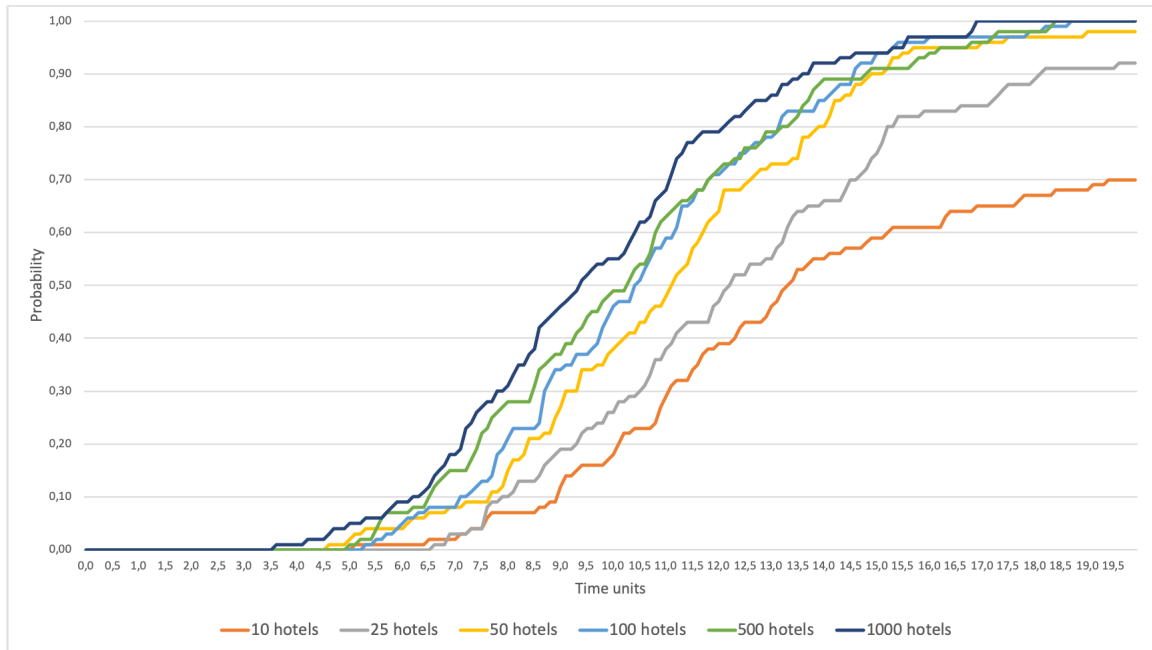

Figure 7: Statistical model-checking of the Travel Booking scenario using jRESP.

invariant and LTL model checkers, and statistical model checking via MULTIVESTA [78] (as done in [79] for a robotic collision avoidance scenario). A further advantage of MISSCEL is that SCEL specifications can be intertwined with (very expressive) raw Maude code. This permits to obtain sophisticated specifications in which SCEL is used to model behaviours, aggregations, and knowledge handling, while scenario-specific details are specified with Maude.

A prototype framework for statistical model-checking [65] has been developed by relying on the simulation data provided by jRESP. The tool, by relying on a randomised algorithm, allows one to verify whether the implementation of a system satisfies a given property with a certain degree of confidence, depending on the number of simulations for a given experiment.

To illustrate in practice the effectiveness of the statistical model-checking facility of jRESP for the verification of SCEL specifications, we have applied it to the SCEL model of the travel booking scenario. In particular, this verification activity aimed at evaluating the probability of reaching within a given deadline a successful execution state, where the customer receives an offer acceptable according to his/her desiderata and completes the booking process with the corresponding hotel. Fig. 7 shows the probability that the successful state is reached within 20 units of time when the number of hotels varies among 10, 25, 50, 100, 500 and 1000. For a given number of hotels, the corresponding experiment has been repeated 100 times, thus ensuring adequate confidence for the precision of the results. The diagram shows 
that the successful state can be reached only after 3.5 units of time and that, as expected, the success probability grows with the number of hotels, although with more than 50 hotels the system performance does not improve significantly.

\subsection{Related work}

SCEL combines the idea of dynamically forming ensembles of agents - getting together to cooperatively work towards some collective goals - which has been extensively analysed $[80,81]$ in the area of distributed artificial intelligence and multi-agent systems [82], with other concepts that have emerged from different research fields of Computer Science and Engineering. Indeed, it borrows from software engineering the importance of component-based design and separation of concerns [83], from multi-agent systems the relevance of knowledge handling and of spatial representation [84, 85, 86, 87, 88], from middleware and network architectures the importance of flexibility in communication [89, 90, 91, 92, 93], from distributed systems' security the role of policies [94], from actors and process algebras the importance of minimality and formality [95, 96].

In the area of concurrency theory, calculi such as those defined in [97] and in [98], relying on the (bio)chemical programming paradigm, have been proposed for the specification of autonomic systems. Some other formalisms, like those introduced in [99] and in [100], aiming at modelling dynamically changing network topologies, also offer linguistic primitives for specifying autonomic systems. Compared to these proposals, SCEL allows one to provide high-level abstract descriptions of systems that nevertheless have a direct correspondence with their implementation.

Summing it up, the main distinctive aspect of SCEL is the actual choice of the specific programming abstractions for autonomic computing and their reconciliation under a single roof with a uniform formal semantics. We refer the interested reader to $[8,65,101]$ for a more complete account of autonomic computing and its relations with SCEL.

\section{AbC: Attribute-based communication}

Collective-Adaptive Systems (CAS) [102] are emerging computational systems, consisting of a massive number of components, featuring complex interaction mechanisms. These systems are usually distributed, heterogeneous, decentralised and interdependent, and are operating in dynamic and often 
unpredictable environments. CAS components combine their behaviours, by forming collectives, to achieve specific goals depending on their attributes, objectives, and functionalities. CAS are inherently scalable and their boundaries are fluid in the sense that components may enter or leave the collective at any time; so they need to dynamically adapt to their environmental conditions and contextual data.

$\mathrm{AbC}$ (Attribute-based Communication calculus, $[103,9,104])$ is a process calculus specifically designed to deal with CAS. It has been heavily inspired by SCEL, but has been designed to reduce complexity and keep the set of linguistic primitives to a minimum. Indeed, it was originally designed as a trimmed version of SCEL that was obtained by ignoring the parts relative to policies and knowledge and concentrating only on behaviours and interfaces. In this respect, $\mathrm{AbC}$ has similar aims to SCELIGHT, but the underlying communication paradigm is very different; explicit message passing for the former and shared memory à la KLAIM for the latter.

Indeed, the original goal of $\mathrm{AbC}$ was to assess the impact of the new message passing paradigm based on attributes and compare it with standard approaches that handle the interaction between distributed components by relying on identities (Actors [105]), or channels ( $\pi$-calculus), or broadcast (B- $\pi$-calculus [106]). In all these formalisms, messages exchanges rely on names or addresses of the involved components and are independent of their status and capabilities. This makes it hard to program, coordinate, and adapt complex behaviours that highly depend on run-time modifications of components.

In $\mathrm{AbC}$, the attribute-based system is however more than just the enabler of the parallel composition of interacting partners; it is also a tool for parameterising system components with respect to the environment or the space where they are executed. Indeed, the environment has a great impact on components behaviour and provides a new means of indirect communication, that allows components to mutually influence each other, also unintentionally.

\subsection{Syntax}

Table 8 contains the syntax of $\mathrm{AbC}$. The top-level entities of the calculus are COMPONEnTs. A component, $\Gamma:_{I} P$, is a process $P$ associated with an attribute environment $\Gamma$, and an interface $I$. The attribute environment provides a collection of attributes whose values represent the status of the component and influence its run-time behaviour. Formally, $\Gamma: \mathcal{A} \rightarrow \mathcal{V}$ is a 


\begin{tabular}{|c|c|c|c|}
\hline \multicolumn{2}{|l|}{ COMPONENTS: } & \multicolumn{2}{|l|}{ PREDICATES: } \\
\hline$C::=\Gamma:_{I} P$ & (component) & $\Pi::=\mathrm{tt}$ & (true) \\
\hline $\mid C_{1} \| C_{2}$ & (composition) & ff & (false) \\
\hline Processes: & & $E_{1} \bowtie E_{2}$ & (comparison) \\
\hline$P::=0$ & (inaction) & $p(\tilde{E})$ & (atomic predicate) \\
\hline | $\Pi(\tilde{x}) \cdot U$ & (attribute-based input) & $\Pi_{1} \wedge \Pi_{2}$ & (conjunction) \\
\hline$(\tilde{E}) @ \Pi . U$ & (attribute-based output) & $\Pi_{1} \vee \Pi_{2}$ & (disjunction) \\
\hline$\langle\Pi\rangle P$ & (context awareness) & $\neg \Pi$ & (negation) \\
\hline$P_{1}+P_{2}$ & (choice) & EXPRESSIONS: & \\
\hline$P_{1} \mid P_{2}$ & (parallel composition) & $E::=v$ & (value) \\
\hline$K$ & (process identifier) & $x$ & (variable) \\
\hline UPDATES: & & $a$ & (attribute identifier) \\
\hline$U::=[a:=E] U$ & (attribute update) & this. $a$ & (local reference) \\
\hline $\mid P$ & (process) & $f(\tilde{E})$ & (operator) \\
\hline
\end{tabular}

Table 8: The syntax of the $\mathrm{AbC}$ calculus

partial map from attribute identifiers $(a \in \mathcal{A})$ to values $(v \in \mathcal{V})$, i.e., to numbers, strings, tuples, ... The interface $I \subseteq \mathcal{A}$ contains the public attributes of a component (the attributes in $\operatorname{dom}(\Gamma)-I$ being private). Composed components $C_{1} \| C_{2}$ are built by using the parallel operator.

A PROCESS $P$ can be: the inactive process 0; the input-prefixed process $\Pi(\tilde{x}) \cdot U$ or the output-prefixed process $(\tilde{E}) @ \Pi . U$, where $U$ is a process preceded by a (possibly empty) sequence of attribute updates; a context aware process, $\langle\Pi\rangle P$, where $\Pi$ is a PREDICATE built from boolean constants and from atomic predicates, based on EXPRESSIONS over attributes, by using standard boolean operators; a nondeterministic choice between two processes, $P_{1}+P_{2}$; a parallel composition of two processes, $P_{1} \mid P_{2}$; or a process call with an identifier $K$ used in a unique process definition $K \triangleq P$.

An expression $E$ may be a constant value $v$, a variable $x$, an attribute name $a$, or a reference this. $a$ to attribute $a$ in the local environment. Predicate $\Pi$ can be either tt, or can be built using comparison operators $\bowtie$ between two expressions and logical connectives $\wedge, \neg, \ldots$. Both expressions and predicates can take more complex forms, of which we deliberately omit the precise syntax; we just refer to them as n-ary operators on subexpressions, i.e., $f(\tilde{E})$ and $p(\tilde{E})$.

\subsection{Informal semantics}

Attribute-based actions for sending and receiving messages permit to establish communication links between different components according to spe- 
cific predicates over their attributes.

Specifically, attribute-based output $(\tilde{E}) @ \Pi$ sends the result of the evaluation of the sequence of expressions $\tilde{E}$ to the components whose attributes satisfy the predicate $\Pi$. Notably, together with the computed values, also the portion of the attribute environment of the sending component that can be perceived by the context is sent; this is obtained from the local environment by limiting its domain to the attributes in the component interface. This information is needed to allow receivers to determine whether they are interested in the sent message.

Instead, attribute-based input $\Pi(\tilde{x})$ specifies receipt of messages from a component satisfying predicate $\Pi$; the sequence $\tilde{x}$ acts as a placeholder for received values. A message can be received when two communication constraints are satisfied: the public local attribute environment satisfies the predicate used by the sender to identify potential receivers, and the sender environment and the communicated message are such that the receiving predicate is satisfied. In this case, attribute updates are performed under the generated substitution.

An attribute update $[a:=E]$ assigns the value of $E$ to the attribute identifier $a$. This action is used to change the values of the attributes according to contextual conditions and to adapt component's behaviour.

The awareness construct $\langle\Pi\rangle P$ blocks the execution of $P$ until predicate $\Pi$ is satisfied when using the local attribute environment, possibly after a change of state by a component. This construct permits to collect awareness data and take decisions based on the changes in the attribute environment.

Attribute updates and awareness predicates are local to components and their executions are atomic with the associated communication action.

\subsection{A taste of the operational semantics}

The operational semantics of $\mathrm{AbC}$ is based on two relations. The transition relation $\mapsto$ that describes the behaviour of individual components and the transition relation $\rightarrow$ that relies on $\longmapsto$ and describes system behaviours.

Relation $\longmapsto$ defines the local behaviour of a component whose labels $\alpha$ have the following format:

$$
\alpha::=\Gamma \triangleright \bar{\Pi}(\tilde{v}) \quad|\quad \Gamma \triangleright \Pi(\tilde{v}) \quad| \widetilde{\Gamma \triangleright \Pi(\tilde{v})}
$$

The $\alpha$-labels $\Gamma \triangleright \bar{\Pi}(\tilde{v})$ and $\Gamma \triangleright \Pi(\tilde{v})$ are used to denote $\mathrm{AbC}$ output and input actions, respectively. The former contains the sender's predicate $\Pi$, that 


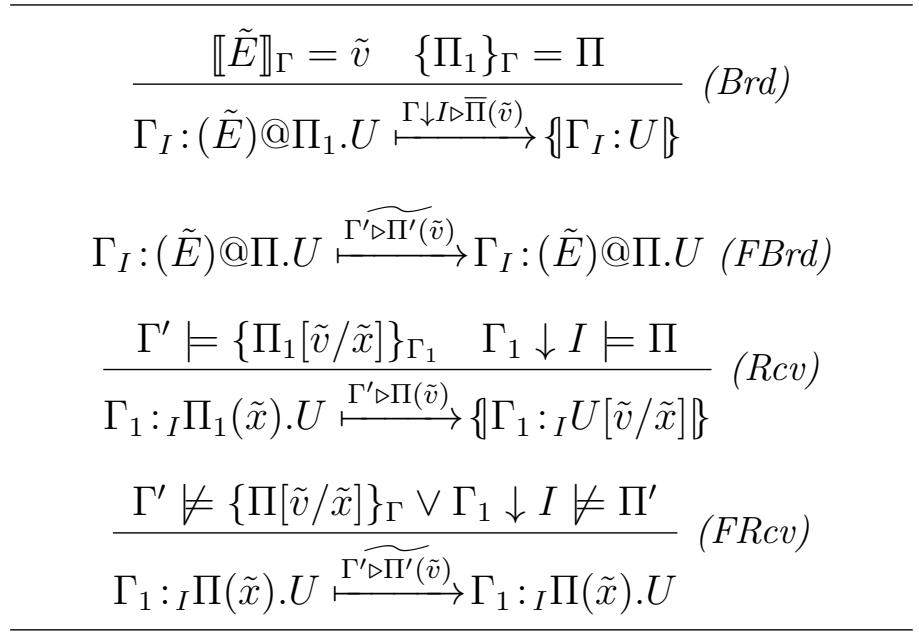

Table 9: Operational Semantics for Components' Communications

specifies the expected communication partners, the transmitted values $\tilde{v}$, and the portion of the sender attribute environment $\Gamma$ that can be perceived by receivers. The latter label is just the complementary label selected among all the possible ones that the receiver may accept. The $\alpha$-labels $\widetilde{\Gamma \triangleright \Pi(\tilde{v})}$ describe the actions exhibited by a component to discard undesired input messages. The contextual label $\Gamma$ indicates the environment in which the components operate and is instrumental to determine whether actual communications can take place at the system level.

The basic rules for components interaction are reported in Table 9. The behaviour of attribute-based output is defined by rule (Brd) in Table 9 . It states that when an output is executed, the sequence of expressions $\tilde{E}$ is evaluated, say to $\tilde{v}$, and the closure $\Pi$ of predicate $\Pi_{1}$ under $\Gamma$ is computed. Hence, these values are sent to other components together with $\Gamma \downarrow I$, i.e., the portion of the attribute environment that can be perceived by the context, obtained from $\Gamma$ by limiting its domain to the attributes in the interface $I$. It has to be noted that rule $(B r d)$ is not sufficient to fully describe output; rule $(F B r d)$ is also needed to model the fact that all incoming messages are discarded when only output actions are possible. Rule (Rcv) governs the execution of input actions. It states that a message can be received when two communication constraints are satisfied: the local attribute environment restricted to interface $I\left(\Gamma_{1} \downarrow I\right)$ satisfies $\Pi$, the predicate used by the sender to identify potential receivers; and the sender environment $\Gamma^{\prime}$ satisfies the 


$$
\begin{array}{lr}
\frac{\Gamma: P \stackrel{\lambda}{\mapsto} \Gamma^{\prime}: P^{\prime}}{\Gamma: P \stackrel{\lambda}{\rightarrow} \Gamma^{\prime}: P^{\prime}} \text { (iComp) } & \frac{\Gamma: P \stackrel{\widetilde{\Pi(\tilde{v})}}{\longrightarrow} \Gamma: P}{\Gamma: P \stackrel{\Pi(\tilde{v})}{\longrightarrow} \Gamma: P} \text { (fComp) } \\
\stackrel{C_{1} \stackrel{\bar{\Pi}(\tilde{v})}{\longrightarrow} C_{1}^{\prime} C_{2} \stackrel{\Pi(\tilde{v})}{\longrightarrow} C_{2}^{\prime}}{\stackrel{\bar{\Pi}(\tilde{v})}{\longrightarrow} C_{1}^{\prime} \| C_{2}^{\prime}} \text { (Com) } & \frac{C_{1} \stackrel{\Pi(\tilde{v})}{\longrightarrow} C_{1}^{\prime} C_{2} \stackrel{\Pi(\tilde{v})}{\longrightarrow} C_{2}^{\prime}}{C_{1}\left\|C_{2} \stackrel{\Pi(\tilde{v})}{\longrightarrow} C_{1}^{\prime}\right\| C_{2}^{\prime}} \text { (Sync) } \\
\hline
\end{array}
$$

Table 10: AbC Communication Rules

receiving predicate $\left\{\Pi_{1}[\tilde{v} / \tilde{x}]\right\}_{\Gamma_{1}}$. When these two constraints are satisfied the input action is performed and the update $U$ is applied under the substitution $[\tilde{v} / \tilde{x}]$. Rule (FRcv) states that an input is discarded when the local attribute environment does not satisfy the sender's predicate, or the receiving predicate is not satisfied by the sender's environment.

The behaviour of an $\mathrm{AbC}$ system is described by means of the transition relation $\rightarrow$ whose labels $\lambda$ are generated by the following grammar:

$$
\lambda::=\bar{\Pi}(\tilde{v}) \quad \mid \quad \Pi(\tilde{v})
$$

The main semantics rules of $\mathrm{AbC}$ systems are reported in Table 10.

Rule (iComp) states that a component evolves with a send $\bar{\Pi}(\tilde{v})$ or receive $\Pi(\tilde{v})$, action (generically denoted by $\lambda$ ) if its internal behaviour (denoted by the relation $\mapsto$ ) allows it. Rule (fComp) states that a component can discard a message $\Pi(\tilde{v})$ if its internal behaviour does not allow the reception of this message by generating the discarding label $\widetilde{\Pi(\tilde{v})}$. Rule (Com) states that if $C_{1}$ evolves to $C_{1}^{\prime}$ by sending a message $\bar{\Pi}(\tilde{v})$ then this message should be delivered to $C_{2}$ which evolves to $C_{2}^{\prime}$ as a result. Note that $C_{2}$ can be also a parallel composition of different components. Thus, rule (Sync) states that multiple components can be delivered the same message in a single transition.

The semantics of the parallel composition operator, in rules (Com) and (Sync) in Table 10, abstracts from the underlying coordination infrastructure that mediates the interactions between components and thus the semantics assumes atomic message exchange. This implies that no component can evolve before the sent message is delivered to all components executing in parallel. Individual components are in charge of using or discarding incoming messages. Message transmission is non-blocking, but reception is not. For 
instance, a component can still send a message even if there is no receiver (i.e., all the target components discard the message); a receive operation can, instead, only take place through synchronisation with an available message.

The original semantics of $\mathrm{AbC}$ outlined above has been formulated in a way that when a component sends a message, that message is delivered to all components in the system in a single move. Atomically, each individual receivers decide whether to use the message or to discard it. This semantics imposes a restriction on the ordering of message delivery because only one component can send its message at a time. Even if this approach is useful to describe the $\mathrm{AbC}$ one-to-many interactions in an abstract way, it is too strong when large scale distributed systems are considered.

To relax the total ordering requirement of the original $\mathrm{AbC}$ semantics, we are currently working on an alternative semantics that takes into account the infrastructure responsible of message dispatching. AbC system are built by using servers of the form $\{\cdot\}^{\iota, \omega}$ that are responsible to manage a set of components. Each server is equipped with an input queue $\iota$ and an output queue $\omega$. The former is the queue of messages that, coming from the environment, the server must deliver to the managed components. The latter is the queue of messages that have been generated locally and that the server must forward to the enclosing system.

\subsection{A travel booking scenario}

In this section we consider the travel agency scenario and outline its $\mathrm{AbC}$ model. We do restrict attention to the part of the scenario concerned with hotel booking, with customers interacting with a broker for room booking. Customers contact the broker that in turn contacts those hotels that expose attributes meeting customers expectations. After receiving pricing and availability information, the broker forwards the best options to customers that then proceeds with the booking directly with the hotel.

The AbC specification of the above scenario relies on three types of components, namely Customer, Broker and Hotel.

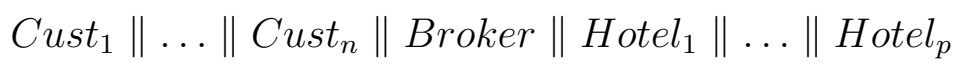

where each component has the following form:

$$
\text { Cust }_{i} \triangleq \Gamma_{i}: P_{C}, \text { Broker } \triangleq \Gamma: P_{B}, \text { Hotel }_{k} \triangleq \Gamma_{k}: P_{H}
$$

with the same behaviour and the same set of attributes names for each type. A UML activity diagram for the $\mathrm{AbC}$ specification of the travel booking 


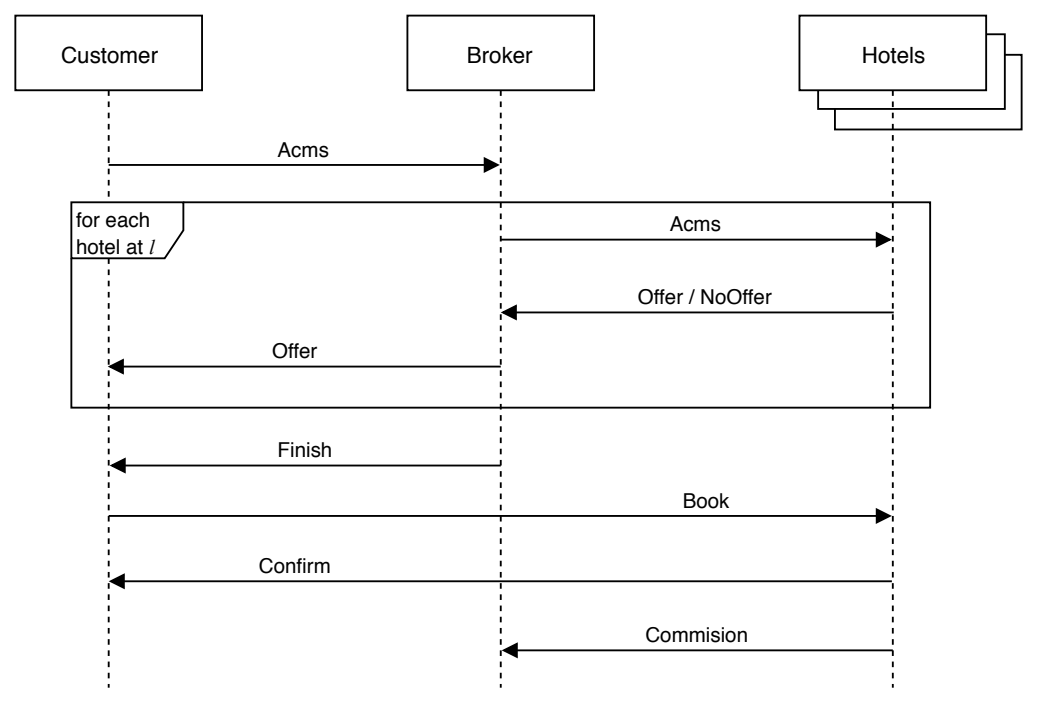

Figure 8: Travel Booking Scenario in AbC: Sequence Diagram.

scenario is reported in Fig. 8. The figure highlights that the broker acts as a mediator between the customer and the hotels and filters the offers according to the customer's preferences. Instead, once the customer has selected the desired hotel, the interactions between them, for booking and confirmation, are not mediated by the broker. In what follows, we provide the specification of the individual components.

Customer. The main attributes of a customer are: id, loc, day, price, dist that represent the customer id, the preferred location, the chosen day, the maximum price, the tolerated distance between the ideal location loc and the actual location of the hotel. In addition, customers use other internal attributes, namely favh - the favorite hotel, ref - the reference broker, and a flag send to control when to send out an inquiry message. The behaviour of a customer is encoded by:

$$
P_{C} \triangleq F \mid A
$$

Process $F$, when enabled by the flag send, proceeds by getting the customer preferences via different getter functions - get_* () - to be used to set up user's preferences. These attribute updates are triggered by exploiting an empty send (a sort of "fake" output action). After setting the preferences, $F$ sends an inquiry to the broker, specified in the sending predicate by (type $=$ "Broker"). The message to be sent includes a tag "acms" for accommodation, 
the location, the booking date and the maximum price the client is prepared to pay. The flag send may be enabled again for another try, if the customer does not find a room at the wanted condition.

$$
\begin{aligned}
F \triangleq & \langle\operatorname{send}\rangle() @(\mathrm{ff}) . \\
& {[\text { day }:=\text { get_day }(), \text { price }:=\text { get_price }(), \text { dist }:=\text { get_dist }(), \operatorname{loc}:=\text { get_loc }()] } \\
& (\text { "acms",this.id,this.loc, this.day,this.price }) @(\text { type }=\text { "Broker").[send := ff] } F
\end{aligned}
$$

Process $A$, instead, handles the replies from the broker. The first branch deals with 'offer' messages associated with hotels located within the preferred distance and at a good price. In such case, a possibly better price $p$ offered by a hotel $h$ made available by the broker with id $b$ are stored in the relevant attributes for later use. The second branch waits for a "finish" message indicating that there is no more offers to be considered, in which case process $A$ continues as $B$.

$$
\begin{aligned}
A \triangleq & (x=\text { "offer" } \wedge \text { this.price } \geq p \wedge \operatorname{diff}(\text { this.loc }, l) \leq \text { this.dist })(x, h, l, p, b) . \\
& {[\text { price }:=\mathrm{p}, \text { favh }:=\mathrm{h}, \text { ref }:=\mathrm{b}] A } \\
& + \\
& (x=\text { "finish" })(x) . B
\end{aligned}
$$

We assume here the availability of function diff that computes the distance between two coordinates.

Upon receipt of the "finish" message, process $B$ makes a decision about how to proceed with the actual booking. Two possibilities are considered: 1. There is no hotels that meets customer's requirement, i.e., (favh $=\perp$ ), then the first branch of $B$ sets 'send' to true for another try.

$$
\begin{aligned}
B \triangleq & \langle\text { favh }=\perp\rangle() @(\mathrm{ff}) .[\text { send }:=\mathrm{tt}] A \\
& + \\
& \langle\text { favh } \neq \perp\rangle(\text { "book", this.id,this.day, this.price, this.ref }) @(\text { id= this.favh }) . \\
& \quad((x=\text { "confirm" })(x) .0 \\
& + \\
& \quad(x=\text { "toolate" })(x) .[\text { send }:=\mathrm{tt}] A)
\end{aligned}
$$

2. A good hotel is found, i.e., (favh $\neq \perp$ ), the process sends a 'book' message attaching the customer id, the wanted date, the paid price and the reference broker to the chosen hotel. If it receives a confirmation afterwards, the session is considered as finished, otherwise, a 'toolate', may arrive meaning that the preferred room in that hotel has already been taken. In that case, a customer may retry again with a new set of preferences by enabling send. 
Broker. The set of attributes of an online broker, $P_{B}$ are id, type, besides the internal attributes $\mathrm{nh}, \mathrm{cnt}$ needed for local computation. $P_{B}$, waits for request messages from its customers and, for each inquiry, creates a handler $H$ to process the inquiry, and spawns a copy of itself for serving future requests:

$$
\begin{gathered}
P_{B} \triangleq(x=\text { "acms" })(x, c, l, d, p) \cdot\left[n h_{l}:=\text { get_hotels }(l), \text { cnt }_{c}:=0\right]\left(H \mid P_{B}\right) \\
H \triangleq(\text { "acms", } c, d, \text { this.id }) @(\text { type }=\text { "Hotel" } \wedge \text { locality }=l) \cdot(A \mid U)
\end{gathered}
$$

Please notice that process $H$ can use the bound variables $c, l, d, p$ and that two internal attributes $\mathrm{nh}_{\mathrm{l}}$ keeping the number of hotels at locality $l$ and $c n t_{c}$ counting the number of replies from hotels are initialised for this session. These attributes can be structured as dynamic vectors, and their slot $k$ can be used for storing relevant information of the session.

Process $H$ forwards the inquiry to all hotels (type = "Hotel") at locality $l$. The message contains the reference customer identity, the required date $d$ and the broker identity id. $H$ then continues as two parallel processes: $A$ dealing with valid offers, $U$ with invalid offers, in addition to sending a termination message.

Process $A$ receives the price-wise acceptable offers from hotels and forwards them to the customer: an offer is forwarded only if the offered price is not greater than the maximum stated price. Furthermore, to handle other messages in parallel, $A$ also spawns a new instance of itself.

$$
\begin{aligned}
& A \triangleq\left\langle c n t_{c}<n h_{l}\right\rangle(x=\text { "offer" } \wedge c=\text { cust } \wedge \text { op } \leq p)(x, \text { cust }, h, l, o p) \cdot(S \mid A) \\
& S \triangleq(\text { "offer", } h, l, o p, \text { this.id }) @(i d=c) .\left[\text { cnt }_{c}:=\text { cnt }_{c}+1\right] 0
\end{aligned}
$$

Process U discards "expensive" offers and ignores 'nooffer' messages; in both cases it increases counter $c n t_{c}$ by 1 . The last branch of $U$ waits until all hotels at the locality $l$ have replied in order to send a 'finish' message to the customer.

$$
\begin{aligned}
U \triangleq & \left\langle c n t_{c}<n h_{l}\right\rangle(x=\text { "offer" } \wedge c=\text { cust } \wedge \text { op }>p)(x, \text { cust }, h, l, o p) . \\
& {\left[c n t_{c}:=c n t_{c}+1\right] U } \\
& + \\
& \left\langle\text { cnt } t_{c}<n h_{l}\right\rangle(x=\text { "nooffer" } \wedge c=\text { cust })(x, \text { cust }) .\left[\text { cnt }_{c}:=c n t_{c}+1\right] U \\
& + \\
& \left.\left\langle\text { cnt } t_{c}=n h_{l}\right\rangle \text { ("finish" }\right) @(i d=c) .0
\end{aligned}
$$


Hotel. The Hotel component has attributes id, type, locality, a list of rooms and their associated price, and a list of trusted brokers blist. Its behaviour is the parallel composition of two subprocesses for dealing with messages from the Broker and Customers components.

$$
P_{H} \triangleq B_{H} \mid C_{H}
$$

On receiving a message from the broker, process $B_{H}$ gets the number of rooms available for the required date, i.e., room $_{d}$, and the corresponding price of the rooms, taking into account the broker identity $b$, i.e., price $e_{d}^{b}$. $B_{H}$ also replicates itself for intercepting other messages while processing the current one using process $A$.

$$
\begin{aligned}
B_{H} \triangleq & (x=\text { "acms" } \wedge b \in \text { this.blist })(x, c, d, b) \cdot\left(A \mid B_{H}\right) \\
A \triangleq & \left.\left\langle\text { room }_{d}>0\right\rangle \text { ("offer", }, \text {, this.id, this.locality, this.price }{ }_{d}^{b}\right) @(i d=b) .0 \\
& + \\
& \left.\left\langle\text { room }_{d}=0\right\rangle \text { ("nooffer", } c\right) @(i d=b) .0
\end{aligned}
$$

$A$ can either reply to the inquiry broker a "offer" or "nooffer" message, depending on the availability of the hotel rooms at day $d$. The "offer" message contains also relevant information such as hotel's id, location and the room price. Moreover, the replies messages carry also a reference of the original request, in our case it is the customer id - $c$.

A hotel may receive booking messages directly from some customers. This type of messages is handled by process $C_{H}$. Specifically, $C_{H}$ reacts to a booking request by using process $R$, in addition to spawn its own copy. A "book" message is processed if it contains a valid broker identifier $b$, i.e., $b \in$ this.blist, which may be used by the hotel to pay a commission. If there are still rooms available at day $d$, the process sends a confirmation to the customer, atomically decrease the number of rooms. In the other case it sends a "toolate" message to the sender of the request and terminates.

$$
\begin{aligned}
C_{H} \triangleq & (x=\text { "book" } \wedge b \in \text { this.blist })(x, c, d, p, b) \cdot\left(R \mid C_{H}\right) \\
R \triangleq & \left\langle\text { room }_{d}>0\right\rangle \text { ("confirm")@ }(i d=c) \cdot\left[\text { room }_{d}:=\text { room }_{d}-1\right] \\
& \quad \text { "comission", } p * 10 \%) @(i d=b) .0 \\
& + \\
& \left\langle\text { room }_{d}=0\right\rangle \text { ("toolate") } @(i d=c) .0
\end{aligned}
$$


Discussion. In our view, the above specification shows that the possibility of using primitives for comparing customer preferences with the features offered by providers greatly simplifies system descriptions and give rise to compact and elegant programs. This also permits to natural handle dynamic changes of demands and offers. Moreover, the minimality of the language and its relatively simple operational semantics makes the specification amenable to formal verification.

\subsection{Programming environment}

Basing the interaction on the values of run-time attributes is indeed a nice idea, but it needs to be supported by a middleware that provides efficient ways for distributing messages, checking attribute values, and updating them. A typical approach is to rely on a centralised broker that keeps track of all components, intercepts every message and forwards it to registered components. It is then the responsibility of each component to decide whether to receive or discard the message. This is the solution proposed in [107] where a Java implementation of $\mathrm{AbC}$ is provided, that however suffers of serious performance problems. Two additional implementations of $\mathrm{AbC}$ have thus been considered, which are built on the top of two well-established programming languages largely used for concurrent programming, namely Erlang and Go, guaranteeing better scalability. The two implementations are called AErlang, for Attribute Erlang, and GoAt, for Go with attributes.

AErlang [108] is a middleware enabling attribute-based communication among programs in Erlang [109], a concurrent functional programming language originally designed for building telecommunication systems and recently successfully adapted to broader contexts, such as large-scale distributed messaging platforms like Facebook and WhatsApp. AErlang lifts Erlang's send and receive communication primitives to attribute-based reasoning. In Erlang, the send primitive requires an explicit destination address while in AErlang processes are not aware of the presence and identity of each other, and communicate using predicates over attributes. AErlang has two main components: (i) a process registry that keeps track of process details, such as the process identifier and the current status, and (ii) a message broker that undertakes the delivery of outgoing messages. The Process registry is a generic server that accepts requests regarding process (un)registration and internal updates. It stores process identifiers and all the information used by the message broker to deliver messages. The Message broker is responsible for delivering messages between processes. It is implemented as an Erlang 
server process listening for interactions from attribute-based send. To address potential bottlenecks arising in the presence of a very large number of processes, the message broker can be set up to run in multiple parallel threads. Like the Java implementation for AbC presented in [107], the message broker is still centralised, however, to avoid broadcasts, the broker has an attribute registry where components register their attribute values and the broker is now responsible for message filtering. Different distribution policies have been implemented that can be used by taking into account dynamicity of attributes and of predicates.

Go $A t^{11}$ extends Go [110], the language introduced by Google to handle massive computation clusters, and to make working in these environments more productive. Go has an intuitive and lightweight concurrency model with a well-understood semantics and extends the CSP model [111] with channel mobility, like in $\pi$-calculus. It also supports buffered channels, to provide mailboxes à la Erlang. The Attribute-based API for Go offers the possibility of using the $\mathrm{AbC}$ primitives to program the interaction of CAS applications directly in Go. The actual implementation faithfully models the formal semantics of $\mathrm{AbC}$ and it is parametric with respect to the infrastructure that mediates interactions. The GoAt API offers the possibility of using three different distributed coordination infrastructures for message exchange, namely cluster, ring, and tree. For all three infrastructures, it has been proved that the message delivery ordering is the same as the one required by the original formal semantics of $\mathrm{AbC}$ [112]. An Eclipse plugin permits programming in a high-level syntax, which can be analysed via formal methods by relying on the operational semantics of $\mathrm{AbC}$. Once the code has been analysed, the GoAt plugin will generate formally verifiable Go code. Examples available from GoAt's site permit to appreciate how intuitive it is to program a complex variant of the well-known problem of Stable Allocation in Content Delivery Network [113].

$A B E L$ is a recently developed programming framework for $\mathrm{AbC}$, implemented in Erlang, that offers a set of APIs with a direct correspondence to the operators used in the $\mathrm{AbC}$ syntax. This direct correspondence allows us to automatically translate $\mathrm{AbC}$ specifications into $A B E L$ terms for actual executions. More details about $A B E L$ can be found in [114]. To provide

\footnotetext{
${ }^{11}$ GoAt codes and examples can be retrieved from https://giulio-garbi.github.io/ goat/.
} 


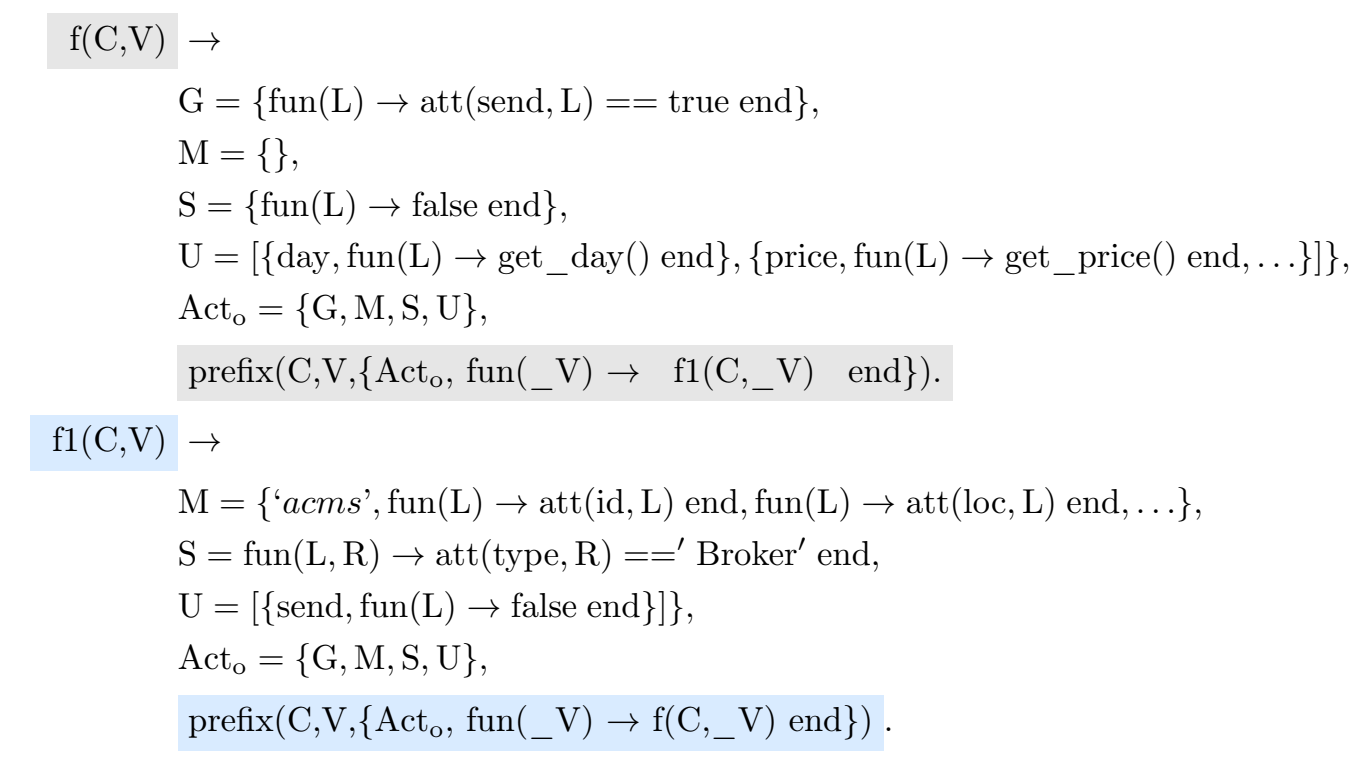

Figure 9: The custmer process $F$ of the travel booking scenario implemented in $A B E L$.

evidence of such a direct correspondence, below we provide some $A B E L$ code snippets that are obtained from the $\mathrm{AbC}$ processes modelling the customer component of the travel booking scenario.

The specification of the process $F$

$$
\begin{aligned}
F \triangleq & \langle\text { send }\rangle() @(f f) .[\text { day }:=\text { get_day }(), \text { price }:=\text { get_price }(), \text { dist }:=\text { get_dist }(), \text { loc }:=\text { get_loc()] } \\
& (\text { "acms",this.id,this.loc, this.day, this.price)@(type = "Broker").[send := ff] }]
\end{aligned}
$$

is translated into $A B E L$ code $^{12}$ in terms of two function definitions that make use of the prefix command, as shown in Fig. 9. An AbC process definition is represented in $A B E L$ through one or more function definitions. As required by the framework, two parameters, namely the address of the hosting component $\mathrm{C}$ and a set of bound variables $\mathrm{V}$ of the executing process are associated with the definitions.

In the code snippet, the definition of $f$ encodes the first part of the process $F$ (the grey area) while that of $f 1$ corresponds to the remaining one (the

\footnotetext{
${ }^{12}$ The $A B E L$ source code for the complete scenario can be downloaded from https: //bitbucket.org/tiezzi/jlamp_survey_code/src/master/AbC/.
} 
blue area). Both definitions have the same structure: first they model an output action and then invoke the prefix command. The output action is characterised by 4 elements: an awareness predicate $G$, a message $M$, a sending predicate $S$ and an attribute update $U$. Their implementation follows the specification of $\mathrm{AbC}$ actions and respects the conventional style of $A B E L$. Thus, a sending predicate $\mathrm{S}$ is implemented as a (boolean-valued) function parametrised with the local (L) and remote $(\mathrm{R})$ environments. The prefix command takes as input an action and a continuation process that states how to proceed upon action termination. In the example, the continuation of $f$ refers to $f 1$, and implements the sequential composition of the two actions, while the continuation of $\mathrm{f} 1$ refers to $f$, thus realising a recursive behaviour.

\subsection{Verification techniques}

Some work has now started to verify properties of $\mathrm{AbC}$ programs. On the one hand, it is under investigation the use of the generic tools that have been designed for verifying properties of Erlang and Go programs. On the other hand, tools are under development to prove directly properties of the $\mathrm{AbC}$ specifications. The second alternative is under consideration because in some cases the correspondence between the actual $\mathrm{AbC}$ specifications and the running programs may not be immediate, and the difference would reduce the effectiveness of the effort.

A novel approach to the analysis of concurrent systems modelled as AbC terms has been introduced in [115]. It relies on the UMC model checker, a tool based on modelling concurrent systems as communicating UML-like state machines [116]. A structural translation from $\mathrm{AbC}$ specifications to the UMC internal format is used as the basis for program analysis. This permits identifying emerging properties of systems and unwanted behaviours. Indeed, we have used the tool outlined in [115] and presented in [117] to translate the $\mathrm{AbC}$ specification of the travel agent scenario into a formalism which is accepted as input by UMC [116]. To make our model concrete we initialised the scenario with two customers, one broker and three hotels. Using the logics ACTL supported by UMC, we were able to specify and verify some orchestration and liveness properties, like:

- A customer that sends an "acms" message will eventually receives a "finish" message

that is expressed by the ACTL formula 


\section{AG [send(Cust, "acms")] AF \{receive(Cust,"finish")\}}

Also other properties like:

- A customer that sends a "book" message will eventually receives either a "confirm" or a "toolate" message

- If a customer receives a "toolate" message, it will react by sending another "acms" message

- If a customer receives a "confirm" message, then the broker will receive a "commission" message

have been verified and have indeed been used to interactively refine the actual specification of the case study.

\subsection{Related work}

In this section, we report on related works concerning languages and calculi with primitives that either model multiparty interactions or provide interesting ways to establish interactions.

Many calculi have been proposed to provide tools for specifying and reasoning about communicating systems. Psi-calculus [118] and its broadcast version [119] are the calculi closest to AbC. Psi-calculus is an extension of the $\pi$-calculus that aims at serving as a meta-theory for process calculi in general. The environment/knowledge is encoded as a special process, named assertion, which influences the behaviour of the process within its scope. The evolution of knowledge is rather complex in Psi-calculus, while the clean separation between knowledge and behaviour in $\mathrm{AbC}$ avoids dependencies between components and enhances readability, compositional reasoning, and maintainability. Broadcast Psi [119] is an extension of Psi-calculus with broadcast primitives whose main communication rule requires that interacting agents take into account the knowledge of each other. Modelling reconfiguration is not easy; it requires modelling different connectivity configurations using assertions and relies on the generation of assertions tagged by a fresh generation number; only the most recent generation is used. A generation becomes obsolete when composed with an assertion from later generation. In

$\mathrm{AbC}$, components can choose to discard messages based on their run time status through the discard rules. 


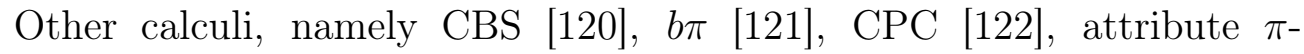
calculus [123], imperative $\pi$-calculus [124], Set-Pi [125] and Broadcast Quality Calculus of [126], share similarities with $\mathrm{AbC}$. We refer the interested reader to [104] for a more detailed account of their relationships with $\mathrm{AbC}$. Indeed, $\mathrm{AbC}$ combines the lessons learnt from the above mentioned calculi, in the sense that $\mathrm{AbC}$ strives for expressivity while aiming at minimality and simplicity. The dynamic settings of attributes and the possibility of inspecting/modifying the environment give $\mathrm{AbC}$ greater flexibility and expressivity while keeping models as much natural as possible.

\section{Concluding remarks}

This paper surveyed four domain-specific coordination languages supporting the engineering of different classes of modern distributed systems. These languages have been developed in the last twenty years by the authors (three of which have been working for quite a while in the Concurrency and Mobility Group at University of Florence) and other collaborators. Within the coordination community other research groups have followed a similar methodology, however relying on different specification models, e.g. coalgebras [127], actors [128] or automata [129], rather than process algebras.

Below, we summarise the programming abstractions introduced with the different formalisms, by also highlighting their main differences, and the lessons learned when designing and using languages for

1. Network-Aware Programming,

2. Service-Oriented Computing,

3. Autonomic Computing,

4. Collective Adaptive Systems Programming.

The design of KLAIM has shown that network awareness in distributed systems can be achieved by the explicit use of localities as first-order citizens of the language. Localities, indeed, identify network nodes, where computation takes place and data is stored. Network awareness relies on the notion of (multiple) tuple spaces, which can be accessed via a unique interface to insert and retrieve data. Communication is thus asynchronous, anonymous and associative, pattern matching plays a crucial role and guarantees high

expressive power. Network awareness paves the way for different kinds of optimisations that take advantage of code mobility, which in KLAIM can rely 
on both static and dynamic scoping disciplines for the interpretation of the locality variables occurring within the mobile code.

From Cows, we learnt that SOC typically abstracts from the structure of the underlying network and from data distribution, both of which become transparent to the programmer. Therefore, differently from Klaim, Cows does not provide code mobility. Although the Cows interaction model has some commonalities with KLAIM's one (both are asynchronous and based on pattern-matching) some of their features are significantly different. Indeed, Cows interactions are triggered by invocations of services along with communication endpoints and use pattern-matching for supporting message correlation. Besides, in SOC a service invocation results either in delivering the message to the corresponding instance or in creating a new instance, but only when no proper instance exists for handling the message. This means that at run time, generally, concurrent instances of the same service can share (part of) their state. The implementation of these mechanisms in Cows relies on the combined use of suitable binder operators and non-standard receive activities that, differently from the KLAIM ones, bind neither names nor variables and exploit pattern-matching to enforce priority among concurrent activities. Two additional distinguishing features of SOC are service persistence, and service fault and termination handling. Cows uses the standard process replication operator for modelling the former, and for modelling the latter relies on the combination of some ingenious constructs to either enforce termination or protect activities in case of termination of concurrent activities .

SCEL was designed by leveraging the experience gained with KLAIM and completely differs from Cows. However, while the syntax of SCEL processes may resemble that of KLAIM ones, their semantics rely on very different mechanisms. In KLAIM, the actions for data management are tagged with the locality where they will take place. In SCEL, instead, they are tagged with a predicate over the attributes in the interface of the components that specifies the ensemble of all those components where the action will take place. Thus, process communication in KLAIM (like in COWs) is always point-to-point, while in SCEL it can also be group-oriented. This feature can be exploited for the formation of components ensembles, which are dynamically created opportunistically and transparently. Moreover, SCEL components are equipped with knowledge repositories that generalise KLAIM's tuple spaces by supporting different knowledge representations and handling mechanisms. Self- and context-awareness make these components capable to 
adapt their behaviour to the evolving needs and the environmental changes. Differently from KLAIM, SCEL does not have constructs for process migration (although a form of mobility can be realised through higher-order communication) but it permits to define policies for regulating processes behaviour which, although they are a parameter of the language, can be fully integrated with its operational semantics.

Finally, $\mathrm{AbC}$ is strongly inspired by SCEL. It refines the group-oriented communication model of SCEL to convey in a distilled form the attributebased communication paradigm to model, program and verify properties of Collective Adaptive Systems. The result of this synthesis effort is a compact calculus, suitable for studying the theoretical impact of the novel communication paradigm and for building new programming frameworks on the top of well-established programming languages, such as Java, Erlang and Go. Compared with SCEL, the knowledge representation in $\mathrm{AbC}$ is abstract and is not designed for detailed reasoning during system evolution. This reflects the different objectives of SCEL and AbC; while SCEL focuses on programming issues, $\mathrm{AbC}$ concentrates on devising a minimal set of primitives to study the effectiveness of attribute-based communication.

To recap, we think that the engineering methodology we presented, as witnessed by the four instantiations we have illustrated, provides a uniform linguistic approach, based on formal methods techniques, for ensuring the trustworthiness of the considered classes of systems and possibly of the other ones that may emerge in the near future. In this respect, we plan to consider the Aggregate Programming [130] domain, where the abstraction level in designing distributed systems further increases. In such an engineering approach, data and devices are aggregated via 'under-the-hood' coordination mechanisms. Although these aggregations resemble the notions of ensemble and collectives discussed in this paper, they mainly focus on distributed computation rather than on communication mechanisms.

Acknowledgements. This work would not have been possible without the contribution of our collaborators that have helped us in shaping the four languages we have introduced. They are too many to be listed, but their names could be inferred from the bibliography below. However, we would like to make an exception and explicitly thank Michele Loreti. Michele has been a driving force for most of the results we have presented, he is not among the authors only because he is one of the PC chairs of the conference to which the work was submitted. The research has been partially supported by the 
MIUR project PRIN 2017FTXR7S "IT-MaTTerS" (Methods and Tools for Trustworthy Smart Systems), and by the MIUR project PRIN 2017TWRCNB "SEDUCE" (Designing Spatially Distributed Cyber-Physical Systems under Uncertainty).

\section{References}

[1] R. De Nicola, G. L. Ferrari, R. Pugliese, F. Tiezzi, A formal approach to the engineering of domain-specific distributed systems, in: COORDINATION, Vol. 10852 of LNCS, Springer, 2018, pp. 110-141. doi:10.1007/978-3-319-92408-3_5.

[2] A. Fuggetta, G. Pietro Picco, G. Vigna, Understanding code mobility, Software Engineering, IEEE Transactions on 24 (1998) 342 - 361. doi:10.1109/32.685258.

[3] M. P. Papazoglou, D. Georgakopoulos, Service-oriented computing, Commun. ACM 46 (10) (2003) 24-28. doi:10.1145/944217.944233.

URL http://doi.acm.org/10.1145/944217.944233

[4] J. O. Kephart, D. M. Chess, The Vision of Autonomic Computing, Computer 36 (2003) 41-50.

[5] S. Anderson, N. Bredeche, A. Eiben, G. Kampis, M. van Steen, Adaptive collective systems: Herding black sheep, BookSprints for ICT Research, 2013.

URL http://booksprints-for-ict-research.eu/wp-content/ uploads/2013/12/bs4ictrsrch-acs.pdf

[6] R. De Nicola, G. L. Ferrari, R. Pugliese, Klaim: A Kernel Language for Agents Interaction and Mobility, IEEE Trans. Software Eng. 24 (5) (1998) 315-330. doi:10.1109/32.685256.

URL https://doi.org/10.1109/32.685256

[7] R. Pugliese, F. Tiezzi, A calculus for orchestration of web services, J. Applied Logic 10 (1) (2012) 2-31.

[8] R. De Nicola, M. Loreti, R. Pugliese, F. Tiezzi, A formal approach to autonomic systems programming: The SCEL language, TAAS 9 (2) (2014) 7. doi:10.1145/2619998.

URL http://doi.acm.org/10.1145/2619998 
[9] Y. Abd Alrahman, R. De Nicola, M. Loreti, On the power of attributebased communication, in: FORTE, LNCS 9688, Springer, 2016, pp. $1-18$.

[10] G. D. Plotkin, A structural approach to operational semantics, J. Log. Algebr. Program. 60-61 (2004) 17-139.

[11] D. Gelernter, Generative communication in linda, ACM Transactions on Programming Languages and Systems 7 (1) (1985) 80-112.

[12] D. Gelernter, Multiple tuple spaces in linda, in: PARLE, Vol. 366 of LNCS, Springer, 1989, pp. 20-27.

[13] N. Davies, S. Wade, A. Friday, G. Blair, L²imbo: a tuple space based platform for adaptive mobile applications, in: ICODP/ICDP, Springer, 1997, pp. 291-302.

[14] S. Castellani, P. Ciancarini, D. Rossi, The ShaPE of ShaDE: a coordination system, Tech. Rep. UBLCS 96-5, Dip. di Scienze dell'Informazione, Univ. Bologna (1996).

[15] D. Deugo, Choosing a Mobile Agent Messaging Model, in: ISADS, IEEE, 2001, pp. 278-286.

[16] G. L. Ferrari, E. Moggi, R. Pugliese, Metaklaim: a type safe multi-stage language for global computing, Mathematical Structures in Computer Science 14 (3) (2004) 367-395. doi:10.1017/S0960129504004165. URL https://doi.org/10.1017/S0960129504004165

[17] R. De Nicola, G. L. Ferrari, R. Pugliese, B. Venneri, Types for access control, Theor. Comput. Sci. 240 (1) (2000) 215-254. doi:10.1016/S0304-3975(99)00232-7. URL https://doi.org/10.1016/S0304-3975(99)00232-7

[18] L. Bettini, M. Loreti, R. Pugliese, Structured Nets in KLAIM, in: SAC, ACM, 2000, pp. 174-180.

[19] L. Bettini, R. De Nicola, G. Ferrari, R. Pugliese, Interactive Mobile Agents in X-Klaim, in: WETICE, IEEE Computer Society Press, 1998, pp. $110-115$. 
[20] L. Bettini, R. De Nicola, R. Pugliese, Klava: a Java package for distributed and mobile applications, Software - Practice and Experience 32 (14) (2002) 1365-1394.

[21] L. Bettini, E. Merelli, F. Tiezzi, X-KLAIM is Back, in: Models, Languages, and Tools for Concurrent and Distributed Programming - Essays Dedicated to Rocco De Nicola on the Occasion of His 65th Birthday, LNCS 11665, Springer, 2019, pp. 115-135.

[22] L. Bettini, Implementing Domain-Specific Languages with Xtext and Xtend, 2nd Edition, Packt Publishing, 2016.

[23] R. De Nicola, M. Loreti, A Modal Logic for Mobile Agents, ACM Trans. Comput. Log. 5 (1) (2004) 79-128. doi:10.1145/963927.963930.

[24] R. De Nicola, D. Gorla, R. Pugliese, Confining data and processes in global computing applications, Sci. Comput. Program. 63 (1) (2006) 57-87. doi:10.1016/j.scico.2005.07.013.

[25] D. Gorla, R. Pugliese, Dynamic management of capabilities in a network aware coordination language, J. Log. Algebr. Program. 78 (8) (2009) 665-689. doi:10.1016/j.jlap.2008.12.001.

[26] R. De Nicola et al., From flow logic to static type systems for coordination languages, Sci. Comput. Program. 75 (6) (2010) 376-397. doi:10.1016/j.scico.2009.07.009.

[27] C. Bodei, P. Degano, G. L. Ferrari, L. Galletta, Tracing where IoT data are collected and aggregated, Logical Methods in Computer Science $13(3)$.

[28] C. Bodei, L. Galletta, Tracking data trajectories in IoT, in: Procs. of International Conference on Information Systems Security and Privacy, SciTePress, 2019, pp. 572-579.

[29] C. Bodei, P. Degano, G. L. Ferrari, L. Galletta, Revealing the trajectories of KLAIM tuples, statically, in: Models, Languages, and Tools for Concurrent and Distributed Programming - Essays Dedicated to Rocco De Nicola on the Occasion of His 65th Birthday, LNCS 11665, Springer, 2019, pp. 437-454. 
[30] C. Bodei, P. Degano, G. L. Ferrari, L. Galletta, Security metric at work on the things of iot systems, LNCS (to appea)r, Springer, 2020.

[31] V. Buravlev, R. De Nicola, C. A. Mezzina, Evaluating the efficiency of linda implementations, Concurrency and Computation: Practice and Experience 30 (8). doi:10.1002/cpe.4381.

URL https://doi.org/10.1002/cpe.4381

[32] G. Ciatto, S. Mariani, M. Louvel, A. Omicini, F. Zambonelli, Twenty Years of Coordination Technologies: State-of-the-Art and Perspectives, in: COORDINATION, Vol. 10852 of LNCS, Springer, 2018, pp. 51-80.

[33] C. Priami, P. Quaglia (Eds.), Global Computing, Vol. 3267 of LNCS, Springer, 2005.

[34] M. Hennessy, J. Riely, Resource access control in systems of mobile agents, Inf. Comput. 173 (1) (2002) 82-120.

[35] C. Fournet, G. Gonthier, J. Lévy, L. Maranget, D. Rémy, A calculus of mobile agents, in: CONCUR '96, Concurrency Theory, 7th International Conference,, Proceedings, Vol. 1119 of Lecture Notes in Computer Science, Springer, 1996, pp. 406-421.

[36] C. Fournet, G. Gonthier, The join calculus: A language for distributed mobile programming, in: Applied Semantics, International Summer School, APPSEM 2000, Advanced Lectures, Vol. 2395 of Lecture Notes in Computer Science, Springer, 2002, pp. 268-332.

[37] L. Cardelli, A. D. Gordon, Mobile ambients, Theor. Comput. Sci. 240 (1) (2000) 177-213.

[38] W3C, Web services activity, https://www.w3.org/2002/ws/, last Access 20 February, 2018.

[39] A. Lapadula, R. Pugliese, F. Tiezzi, A Calculus for Orchestration of Web Services., in: ESOP, Vol. 4421 of LNCS, Springer, 2007, pp. 3347.

[40] OASIS WSBPEL TC, Web Services Business Process Execution Language Version 2.0., Tech. rep., OASIS (April 2007). 
[41] M. Merro, D. Sangiorgi, On asynchrony in name-passing calculi., Mathematical Structures in Computer Science 14 (5) (2004) 715-767.

[42] R. Amadio, I. Castellani, D. Sangiorgi, On Bisimulations for the Asynchronous pi-Calculus, Theor. Comput. Sci. 195 (2) (1998) 291-324.

[43] L. Cesari, R. Pugliese, F. Tiezzi, Blind-date conversation joining, Service Oriented Computing and Applications 11 (3) (2017) 265-283.

[44] R. Milner, J. Parrow, D. Walker, A Calculus of Mobile Processes, I and II, Inf. Comput. 100 (1) (1992) 1-40, 41-77.

[45] A. Lapadula, R. Pugliese, F. Tiezzi, Using formal methods to develop WS-BPEL applications, Sci. Comput. Program. 77 (3) (2012) 189-213.

[46] L. Cesari, R. Pugliese, F. Tiezzi, A tool for rapid development of WSBPEL applications, SIGAPP Applied Computing Review 11 (1) (2010) $27-40$.

[47] A. Lapadula, R. Pugliese, F. Tiezzi, Regulating data exchange in service oriented applications, in: FSEN, Vol. 4767 of LNCS, Springer, 2007, pp. 223-239.

[48] R. Pugliese, F. Tiezzi, N. Yoshida, On Observing Dynamic Prioritised Actions in SOC, in: ICALP, Vol. 5556 of LNCS, Springer, 2009, pp. $558-570$.

[49] A. Fantechi et al., A logical verification methodology for serviceoriented computing, ACM Trans. Softw. Eng. Methodol. 21 (3) (2012) $16: 1-16: 46$.

[50] M. Masi, R. Pugliese, F. Tiezzi, On secure implementation of an IHE XUA-based protocol for authenticating healthcare professionals, in: ICISS, Vol. 5905 of LNCS, Springer, 2009, pp. 55-70.

[51] S. Gnesi, R. Pugliese, F. Tiezzi, The sensoria approach applied to the finance case study, in: Rigorous Software Engineering for ServiceOriented Systems, Vol. 6582 of LNCS, Springer, 2011, pp. 698-718. 
[52] A. Lapadula, R. Pugliese, F. Tiezzi, tcows: A timed service-oriented calculus, in: Proc. of 4th International Colloquium on Theoretical Aspects of Computing (ICTAC), Vol. 4711 of LNCS, Springer, 2007, pp. 275-290.

[53] A. Lapadula, R. Pugliese, F. Tiezzi, Service discovery and negotiation with Cows, in: Proc. of 3rd International Workshop on Automated Specification and Verification of Web Systems (WWV), Vol. 200(3) of ENTCS, Elsevier Science, 2008, pp. 133-154.

[54] D. Prandi, P. Quaglia, Stochastic Cows, in: Proc. of 5th International Conference on Service Oriented Computing (ICSOC), Vol. 4749 of LNCS, Springer, 2007, pp. 245-256.

[55] D. Prandi, P. Quaglia, N. Zannone, Formal analysis of BPMN via a translation into Cows, in: Proc. of 10th international conference on Coordination Models and Languages (COORDINATION), Vol. 5052 of LNCS, Springer, 2008, pp. 249-263.

[56] C. Laneve, G. Zavattaro, Foundations of Web Transactions, in: Proc. of 8th International Conference on Foundations of Software Science and Computational Structures (FoSSaCS), Vol. 3441 of LNCS, Springer, 2005, pp. 282-298.

[57] C. Laneve, G. Zavattaro, web-pi at Work, in: Prof. of 1st International Symposium on Trustworthy Global Computing (TGC), Vol. 3705 of LNCS, Springer, 2005, pp. 182-194.

[58] M. Mazzara, I. Lanese, Towards a Unifying Theory for Web Services Composition, in: Proc. of 3rd International Workshop on Web Services and Formal Methods (WS-FM), Vol. 4184 of LNCS, Springer, 2006, pp. $257-272$.

[59] M. Mazzara, R. Lucchi, A pi-calculus based semantics for WS-BPEL, Logic and Algebraic Programming 70 (1) (2006) 96-118.

[60] M. Butler, C. Hoare, C. Ferreira, A Trace Semantics for Long-Running Transactions, in: 25 Years Communicating Sequential Processes, Vol. 3525 of LNCS, Springer, 2005, pp. 133-150. 
[61] M. Boreale, R. Bruni, L. Caires, R. De Nicola, I. Lanese, M. Loreti, F. Martins, U. Montanari, A. Ravara, D. Sangiorgi, V. Vasconcelos, G. Zavattaro, SCC: a Service Centered Calculus., in: Proc. of 3rd International Workshop on Web Services and Formal Methods (WSFM)), Vol. 4184 of LNCS, Springer, 2006, pp. 38-57.

[62] M. Boreale, R. Bruni, R. De Nicola, M. Loreti, Sessions and Pipelines for Structured Service Programming, in: Proc. of 10th International Conference on Formal Methods for Open Object-based Distributed Systems (FMOODS), Vol. 5051 of LNCS, Springer, 2008, pp. 19-38.

[63] C. Guidi, R. Lucchi, R. Gorrieri, N. Busi, G. Zavattaro, SOCK: A Calculus for Service Oriented Computing, in: ICSOC, Vol. 4294 of LNCS, Springer, 2006, pp. 327-338.

[64] M. Wirsing, M. M. Hölzl (Eds.), Rigorous Software Engineering for Service-Oriented Systems - Results of the SENSORIA Project on Software Engineering for Service-Oriented Computing, Vol. 6582 of LNCS, Springer, 2011.

[65] R. De Nicola, D. Latella, A. Lluch-Lafuente, M. Loreti, A. Margheri, M. Massink, A. Morichetta, R. Pugliese, F. Tiezzi, A. Vandin, The SCEL language: Design, implementation, verification, in: Software Engineering for Collective Autonomic Systems - The ASCENS Approach, LNCS 8998, Springer, 2015, pp. 3-71.

[66] L. Cesari, R. De Nicola, R. Pugliese, M. Puviani, F. Tiezzi, F. Zambonelli, Formalising adaptation patterns for autonomic ensembles, in: Formal Aspects of Component Software - 10th International Symposium, FACS 2013, LNCS 8348, Springer, 2013, pp. 100-118.

[67] G. Cabri, N. Capodieci, L. Cesari, R. De Nicola, R. Pugliese, F. Tiezzi, F. Zambonelli, Self-expression and dynamic attribute-based ensembles in SCEL, in: Leveraging Applications of Formal Methods, Verification and Validation. Technologies for Mastering Change - 6th International Symposium, ISoLA 2014, LNCS 8802, Springer, 2014, pp. 147-163.

[68] L. Cesari et al., Formalising Adaptation Patterns for Autonomic Ensembles, in: FACS, Vol. 8348 of LNCS, Springer, 2013, pp. 100-118. 
[69] T. Bures et al., A life cycle for the development of autonomic systems: The e-mobility showcase, in: SASOW, IEEE, 2013, pp. 71-76.

[70] R. De Nicola, G. Ferrari, M. Loreti, R. Pugliese, A Language-based Approach to Autonomic Computing, in: FMCO, LNCS 7542, Springer, 2012, pp. 25-48.

[71] A. Margheri, R. Pugliese, F. Tiezzi, Linguistic abstractions for programming and policing autonomic computing systems, in: UIC/ATC, IEEE, 2013, pp. 404-409.

[72] P. Mayer et al., The Autonomic Cloud: A vision of voluntary, peer-2peer cloud computing, in: SASOW, IEEE, 2013, pp. 89-94.

[73] U. Montanari, R. Pugliese, F. Tiezzi, Programming autonomic systems with multiple constraint stores, in: Software, Services, and Systems, LNCS 8950, Springer, 2015, pp. 641-661.

[74] R. De Nicola, A. Lluch-Lafuente, M. Loreti, A. Morichetta, R. Pugliese, V. Senni, F. Tiezzi, Programming and verifying component ensembles, in: From Programs to Systems. The Systems perspective in Computing - ETAPS Workshop, FPS 2014, LNCS 8415, Springer, 2014, pp. 69-83.

[75] A. Margheri, M. Masi, R. Pugliese, F. Tiezzi, A rigorous framework for specification, analysis and enforcement of access control policies, IEEE Trans. Software Eng. 45 (1) (2019) 2-33. doi:10.1109/TSE.2017.2765640.

URL https://doi.org/10.1109/TSE. 2017.2765640

[76] G. J. Holzmann, The model checker SPIN, IEEE Trans. Softw. Eng. 23 (5) (1997) 279-295.

[77] M. Clavel, F. Durán, S. Eker, P. Lincoln, N. Martí-Oliet, J. Meseguer, C. L. Talcott, All About Maude, Vol. 4350 of LNCS, Springer, 2007.

[78] S. Sebastio, A. Vandin, MultiVeStA: statistical model checking for discrete event simulators, in: ValueTools, ICST/ACM, 2013, pp. 310-315.

[79] L. Belzner, R. De Nicola, A. Vandin, M. Wirsing, Reasoning (on) service component ensembles in rewriting logic, in: Specification, Algebra, and Software, LNCS 8373, Springer, 2014, pp. 188-211. 
[80] E. Durfee, V. Lesser, D. D. Corkill, Trends in cooperative distributed problem solving, Knowledge and Data Engineering, IEEE Transactions on 1 (1) (1989) 63-83.

[81] M. Klusch, A. Gerber, Dynamic coalition formation among rational agents, IEEE Intelligent Systems 17 (3) (2002) 42-47. doi:http://doi.ieeecomputersociety.org/10.1109/MIS.2002.1005630.

[82] F. Zambonelli, A. Omicini, Challenges and research directions in agentoriented software engineering, Autonomous Agents and Multi-Agent Systems 9 (3) (2004) 253-283.

[83] P. McKinley, S. Sadjadi, E. Kasten, B. H. C. Cheng, Composing adaptive software, Computer 37 (7) (2004) 56-64.

[84] A. S. Rao, AgentSpeak(L): BDI Agents Speak Out in a Logical Computable Language, in: MAAMAW, LNCS 1038, Springer, 1996, pp. $42-55$.

[85] R. H. Bordini, J. F. Hübner, R. Vieira, Jason and the Golden Fleece of Agent-Oriented Programming, in: Multi-Agent Programming, Vol. 15 of Multiagent Systems, Artificial Societies, and Simulated Organizations, Springer, 2005, pp. 3-37.

[86] M. Winikoff, Jack ${ }^{\mathrm{tm}}$ intelligent agents: An industrial strength platform, in: Multi-Agent Programming, Vol. 15 of Multiagent Systems, Artificial Societies, and Simulated Organizations, Springer, 2005, pp. 175-193.

[87] F. L. Bellifemine, G. Caire, D. Greenwood, Developing Multi-Agent Systems with JADE, Wiley Series in Agent Technology, John Wiley \& Sons, 2007.

[88] M. Dastani, 2APL: a practical agent programming language, Autonomous Agents and Multi-Agent Systems 16 (3) (2008) 214-248.

[89] L. Mottola, G. P. Picco, Logical Neighborhoods: A Programming Abstraction for Wireless Sensor Networks, in: DCOSS, LNCS 4026, Springer, 2006, pp. 150-168. 
[90] P. Costa, L. Mottola, A. L. Murphy, G. Picco, Tuple Space Middleware for Wireless Networks, in: Middleware for Network Eccentric and Mobile Applications, Springer, 2009, pp. 245-264.

[91] M. Mamei, F. Zambonelli, Programming pervasive and mobile computing applications: The tota approach, ACM Trans. Softw. Eng. Methodol. 18 (4).

[92] E. Nordström, P. Gunningberg, C. Rohner, A search-based network architecture for mobile devices, Tech. Rep. 2009-003, Uppsala University, Computer Systems (2009).

[93] L. Mottola, G. P. Picco, Middleware for wireless sensor networks: an outlook, J. Internet Services and Applications 3 (1) (2012) 31-39.

[94] NIST, A survey of access control models, http://csrc. nist.gov/news_events/privilege-management-workshop/ PvM-Model-Survey-Aug26-2009.pdf (2009).

[95] G. A. Agha, ACTORS - a model of concurrent computation in distributed systems, MIT Press series in artificial intelligence, MIT Press, 1990 .

[96] R. Milner, Communication and concurrency, PHI Series in computer science, Prentice Hall, 1989.

[97] J.-P. Banâtre, Y. Radenac, P. Fradet, Chemical Specification of Autonomic Systems, in: IASSE, ISCA, 2004, pp. 72-79.

[98] O. Andrei, H. Kirchner, A higher-order graph calculus for autonomic computing, in: Graph Theory, Computational Intelligence and Thought, Springer, 2009, pp. 15-26.

[99] N. Mezzetti, D. Sangiorgi, Towards a calculus for wireless systems, Electr. Notes Theor. Comput. Sci. 158 (2006) 331-353.

[100] A. Singh, C. R. Ramakrishnan, S. A. Smolka, A process calculus for mobile ad hoc networks, Sci. Comput. Program. 75 (6) (2010) 440-469.

[101] M. Wirsing, M. M. Hölzl, N. Koch, P. Mayer (Eds.), Software Engineering for Collective Autonomic Systems - The ASCENS Approach, Vol. 8998 of LNCS, Springer, 2015. 
[102] A. Ferscha, Collective adaptive systems, in: UbiComp/ISWC, ACM, 2015, pp. 893-895.

[103] Y. Abd Alrahman et al., A calculus for attribute-based communication, in: SAC '15, ACM, 2015, pp. 1840-1845. doi:10.1145/2695664.2695668. URL http://doi.acm.org/10.1145/2695664.2695668

[104] Y. A. Alrahman, R. De Nicola, M. Loreti, A calculus for collectiveadaptive systems and its behavioural theory, Inf. Comput. 268.

[105] G. Agha, Actors: A Model of Concurrent Computation in Distributed Systems, MIT Press, Cambridge, MA, USA, 1986.

[106] K. V. S. Prasad, A calculus of broadcasting systems, Sci. Comput. Program. 25 (2-3) (1995) 285-327.

[107] Y. Abd Alrahman, R. De Nicola, M. Loreti, Programming of CAS systems by relying on attribute-based communication, in: ISoLA, Vol. 9952 of LNCS, Springer, 2016, pp. 539-553.

[108] R. De Nicola, T. Duong, O. Inverso, C. Trubiani, AErlang: Empowering erlang with attribute-based communication, in: COORDINATION, Vol. 10319 of LNCS, Springer, 2017, pp. 21-39.

[109] Ericsson Computer Science Laboratory, The Erlang programming language, https://www.erlang.org/, last Access April 12, 2018.

[110] Google, The Go programming language, https://golang.org/doc/, last Access February 20, 2018.

[111] C. A. R. Hoare, Communicating sequential processes, Communications of the ACM 21 (8) (1978) 666-677.

[112] Y. Abd Alrahman, R. De Nicola, G. Garbi, M. Loreti, A distributed coordination infrastructure for attribute-based interaction, in: Formal Techniques for Distributed Objects, Components, and Systems - 38th IFIP WG 6.1 International Conference, FORTE 2018, LNCS 10854, Springer, 2018, pp. 1-20.

[113] B. M. Maggs, R. K. Sitaraman, Algorithmic nuggets in content delivery, SIGCOMM Comput. Commun. Rev. 45 (3) (2015) 52-66. 
doi:10.1145/2805789.2805800.

URL http://doi.acm.org/10.1145/2805789.2805800

[114] R. De Nicola, T. Duong, M. Loreti, ABEL - A domain specific framework for programming with attribute-based communication, in: Coordination Models and Languages, COORDINATION 2019, Vol. 11533 of Lecture Notes in Computer Science, Springer, 2019, pp. 111-128. doi:10.1007/978-3-030-22397-7_7.

URL https://doi.org/10.1007/978-3-030-22397-7\_7

[115] R. De Nicola, T. Duong, O. Inverso, F. Mazzanti, Verifying properties of systems relying on attribute-based communication, in: ModelEd, TestEd, TrustEd, Vol. 10500 of LNCS, Springer, 2017, pp. 169-190.

[116] M. H. ter Beek, A. Fantechi, S. Gnesi, F. Mazzanti, A state/event-based model-checking approach for the analysis of abstract system properties, Sci. Comput. Program. 76 (2) (2011) 119-135.

[117] T. Duong, Programming and verifying systems relying on attributebased communication, Phd thesis, GSSI - School of Advanced Studies, forthcoming (2019).

[118] J. Bengtson, M. Johansson, J. Parrow, B. Victor, Psi-calculi: a framework for mobile processes with nominal data and logic, Logical Methods in Computer Science 7 (1). doi:10.2168/LMCS-7(1:11)2011.

URL https://doi.org/10.2168/LMCS-7(1:11)2011

[119] J. Borgström, S. Huang, M. Johansson, P. Raabjerg, B. Victor, J. A. Pohjola, J. Parrow, Broadcast psi-calculi with an application to wireless protocols, Software and System Modeling 14 (1) (2015) 201-216. doi:10.1007/s10270-013-0375-z. URL https://doi.org/10.1007/s10270-013-0375-z

[120] K. V. Prasad, A calculus of broadcasting systems, Sci. Comput. Program. 25 (2) (1995) 285-327.

[121] C. Ene, T. Muntean, A broadcast-based calculus for communicating systems, in: Parallel and Distributed Processing Symposium, International, Vol. 3, IEEE Computer Society, 2001, pp. 30149b-30149b. 
[122] T. Given-Wilson, D. Gorla, B. Jay, Concurrent pattern calculus, in: Theoretical Computer Science, Springer, 2010, pp. 244-258.

[123] M. John, C. Lhoussaine, J. Niehren, A. M. Uhrmacher, The attributed pi-calculus with priorities, in: Transactions on Computational Systems Biology XII, Springer, 2010, pp. 13-76.

[124] M. John, C. Lhoussaine, J. Niehren, Dynamic compartments in the imperative $\pi$-calculus, in: Computational Methods in Systems Biology, Springer, 2009, pp. 235-250.

[125] A. Bruni, S. Modersheim, F. Nielson, H. R. Nielson, Set-pi: Set membership p-calculus, in: 2015 IEEE 28th Computer Security Foundations Symposium, 2015, pp. 185-198. doi:10.1109/CSF.2015.20.

[126] R. Vigo, F. Nielson, H. Riis Nielson, Broadcast, Denial-of-Service, and Secure Communication, in: 10th International Conference on integrated Formal Methods (iFM'13), Vol. 7940 of LNCS, 2013, pp. 410427.

[127] F. Arbab, J. J. M. M. Rutten, A coinductive calculus of component connectors, in: WADT, Vol. 2755 of LNCS, Springer, 2002, pp. 34-55.

[128] M. Sirjani, A. Movaghar, A. Shali, F. S. de Boer, Modeling and verification of reactive systems using rebeca, Fundamenta Informaticae 63 (4) (2004) 385-410.

[129] C. Baier, M. Sirjani, F. Arbab, J. J. M. M. Rutten, Modeling component connectors in reo by constraint automata, Sci. Comput. Program. 61 (2) (2006) 75-113. doi:10.1016/j.scico.2005.10.008. URL https://doi.org/10.1016/j.scico.2005.10.008

[130] J. Beal, M. Viroli, Aggregate programming: From foundations to applications, in: SFM, Vol. 9700 of LNCS, Springer, 2016, pp. 233-260. 